\title{
SOFT COLLECTIVE EXCITATIONS IN HOT GAUGE THEORIES
}

\author{
Jean-Paul BLAIZOT and Edmond IANCU \\ Service de Physique Théorique', CE-Saclay \\ 91191 Gif-sur-Yvette, France
}

18 June 1993

\begin{abstract}
The longwavelength excitations of a quark-gluon plasma at high temperature can be described as collective oscillations of gauge and fermionic average fields. We show that, at leading order in the coupling strength, the Dyson-Schwinger equations for the $N$-point functions reduce to a set of coupled equations for these average fields and their induced sources which involve only 2-point functions. The equations for the 2-point functions describe the dynamics of the hard plasma particles in the presence of soft background fields. They may be given the form of simple kinetic equations. Both the wavelength and the amplitude of the collective modes are controlled by the coupling strength, and we show that it is important to take this properly into account in order to obtain consistent equations of motion which are covariant under gauge transformations. By solving the kinetic equations for the 2point functions with well defined (i.e. retarded or advanced) boundary conditions, we obtain the induced currents in closed forms. These act as generating functionals for the one-particle irreducible amplitudes with soft external lines, and yield in particular all the so called "hard thermal loops" identified in the diagrammatic analysis.
\end{abstract}

$\mathrm{SPhT} / 93-064$

Submitted to Nuclear Physics B

PACS No: 12.38.Mh, 12.38.Bx, 52.25.Dg, 11.15.Kc

\footnotetext{
${ }^{*} \mathrm{CNRS}$

†Laboratoire de la Direction des Sciences de la Matière du Commissariat à l'Energie Atomique
} 


\section{Introduction}

The study of the long wavelength excitations of the quark-gluon plasma is interesting in several respects. In particular, it provides clues to basic features of the plasma offequilibrium dynamics, and it is a first step towards a full description of its transport properties. Interesting progress in this area has been made recently, and consistent schemes to perform calculations of amplitudes in hot gauge theories have begun to emerge [1-6], giving rise to various applications [7-11].

We consider in this paper a quark-gluon plasma in thermal equilibrum at high temperature $T$ well beyond the critical temperature for the deconfining phase transition, and neglect quark masses. Then, the only energy scale in the problem is $T$. We also assume a regime where the effective coupling constant $g$ is small. In the plasma, quarks and gluons have typical energies and momenta of order $T$, and will be referred to as "hard" particles; their number density is proportional to $T^{3}$. Thus, the typical interparticle distance is of the order $1 / T$, that is, of the order of the thermal wavelength $\lambda_{T}$. This means, for instance, that the Pauli principle cannot be ignored for the hard particles. However, when coupled to weak and slowly varying disturbances, the plasma may develop a collective behaviour on a typical length/time scale of order $1 / g T$. A familiar example of such a collective behaviour is Debye's screening. Collectivity arises because any motion taking place over a distance scale $1 / g T$ may involve coherently a large number of hard particles. We shall see later that such collective motions can be treated as classical degrees of freedom.

The existence of such possible excitations manifests itself in specific difficulties encountered in perturbative calculations. In particular, diagrammatic analyses have revealed that amplitudes with "soft" external lines, i.e. with all external momenta of order $g T$, may receive contributions from an infinite number of loop diagrams which are as large as the tree amplitudes [13, 14, 15, 3, 2]. These contributions arise from one-loop integrals in which the loop momentum is hard, and for this reason they have been named "hard thermal loops" (HTL). As they are of the same order of magnitude as the tree amplitudes, they have to be resummed in consistent higher order calculations [1], 2].

The need for resummation in a perturbative expansion usually points to simple underlying physics, as shown, for instance, by the classic example of the electron gas. There, the resummation of ring diagrams performed by Brueckner and Gell-Mann [20] reflects the simple physical phenomenon of Debye's screening. In order to illuminate the simple physics underlying the resummation needed in QCD, we have initiated [16, 17] a new approach to the problem, by working directly at the level of the equation of motion rather than on the Feynman diagrams. The crucial remark is that the collective phenomena that 
we are trying to describe involve mean fields which represent the average motion of hard particles over distances large compared to their mean separation. Such mean fields may be induced by external disturbances, but they are also the natural degrees of freedom describing the soft normal modes of the quark-gluon plasma. Our main objective will be therefore to obtain consistent equations of motions for these mean fields. In order to allow for collective excitations with arbitrary quantum numbers, we shall consider both fermionic and bosonic average fields. The physical picture which will emerge from our analysis is that the dominant interactions in the plasma are those between the mean fields and the hard particles. Direct interactions among hard particles can be neglected, but since hard particles can contribute coherently, they strongly affect the dynamics of the mean fields. Our final equations will take the form of a coupled set of mean field equations and kinetic equations describing the motion of hard particles in the presence of the mean fields. These equations generalize the familiar set of Vlasov and field equations of ordinary electromagnetic plasmas [18, 19].

When dealing with this type of problems one is usually facing three kinds of approximations, involving, respectively, the strength of the coupling, the amplitude of the oscillation, and the wavelength or period of the modes that one is studying. In the present case, there is no other scale than the temperature, and it turns out that all three approximations are controlled by the single small parameter, $g$. They should be viewed, then, as three facets of the same approximation, namely an expansion in powers of $g$. Thus, the collective modes with wavelength $\sim 1 / g T$, have, as we shall see, amplitudes limited by $\bar{\psi} \psi \lesssim g T^{3}$ and $F_{\mu \nu} \lesssim g T^{2}$, respectively. In order to preserve gauge covariance, it is essential that these three features of the approximation scheme be implemented together consistently. The naive loop expansion fails precisely because it takes care only of the first facet, namely the expansion in powers of the interaction vertices.

As alluded to before, one outcome of the present analysis is a set of gauge covariant kinetic equations for hard quarks and gluons moving in soft gauge and fermionic fields. We thus make contact with previous works on the kinetic theory of non abelian plasmas [21-25]. However, the special role of the scale $g T$, as a unifying parameter controlling the various approximations being done in deriving kinetic equations, has never been properly recognized. Because they include terms which are found to be of different orders in our expansion scheme, most kinetic equations proposed so far are not only more complicated, they are also inconsistent. Besides, although quite some effort was spent in using gauge invariant Wigner functions, specific issues related to gauge invariance in a quantum theory, such as gauge fixing independence, or the elimination of ghost particles, have never been properly addressed. The resulting inconsistencies can be seen in calculating one-particle 
irreducible amplitudes which, in leading order, can be deduced from the kinetic equations. As well known, these amplitudes satisfy Ward identities imposed by gauge symmetry. This puts constraints on the structure of the non-linear terms which enter the kinetic equations, and such constraints do not appear to be satisfied by the transport equations proposed so far.

Our strategy for deriving the relevant equations of motion is similar to that already used in connection with the abelian case [16]. However, the structure of the non abelian theory is much richer, and the present work contains many new technical developments. It is organized as follows. In Sec.2, after summarizing the conventions and the notations used in this paper, we write the equations of motion relating the average fields to the off-equilibrium 2-point functions. The connected parts of these 2-point functions play the role of induced sources for the average fields. From them, one-particle irreducible amplitudes for the soft fields can be calculated.

The equations for the average fields constitute the first level in the hierarchy of Dyson-Schwinger equations for the $N$-point functions. In Sec.3, we describe all the approximations which allow us to calculate the 2-point functions. In leading order, these functions concern only hard particles, for which radiative corrections can be neglected. This implies that the hierarchy truncates at the level of 2-point functions. We analytically continue the 2-point functions to real time, and we implement the condition that the average fields are slowly varying with the help of Wigner functions and gradient expansions. We also take into account the fact that the strength of the mean fields have to be constrained so as to obtain consistent equations. We emphasize that this does not result in a trivial linearization of the equations since, for example, gauge fields involved in covariant derivatives are to be kept to all orders. At the end of Sec.3, we have a set of gauge covariant equations for the induced pieces of the 2-point functions. These equations incorporate consistently all leading order effects in $g$.

In Sec.4, the equations for the 2-point functions are transformed into equations for the induced sources. These describe the polarizability of the plasma at leading order in $g$. They involve only the physical hard particles from the thermal bath, i.e. the quarks, the antiquarks and the transverse gluons. The equations are covariant under gauge transformations of the mean fields. They reduce to simple kinetic equations for density matrices which, in general, are non diagonal in color space, or which can mix boson and fermion degrees of freedom. These density matrices play the role of generalized classical distribution functions for the hard particles. The kinetic equations are solved for retarded boundary conditions. We get then explicit expressions for the induced sources in terms of the average fields. 
The knowledge of these induced sources entirely determines the dynamics of the average fields, which is discussed in Sec.5. There we obtain all one-particle irreducible amplitudes for soft fields by functional differentiation of the induced sources. We recover in this way all the "hard thermal loops", with the boundary conditions appropriate to the problem at hand, i.e. the causal propagation of the mean fields. The Ward identities satisfied by the HTL are seen to follow simply from the conservation laws obeyed by the induced currents. In cases where Landau damping is inoperative, a generating functional for the induced currents is obtained. It may be regarded as the effective action which describes the propagation of long wavelength fields in a polarizable non abelian plasma, and coincides with the generating functional for hard thermal loops previously derived in [26, 27, 28]. Properties of this generating functional are also discussed in [29, 30].

The main conclusions are summarized in Sec.6. Technical details related to the use of a general covariant gauge in our main discussion are gathered in Appendices A and B. In App.A we show that, in leading order, ghost particles do not undergo collective motion. This is in line with our conclusions concerning the semiclassical character of the leading order polarization phenomena. This same conclusion is supported by the independence of our results with respect to the choice of the gauge in the original quantum theory. This is proved in App.B. In App.C, we clarify the conditions under which the effective action proposed in Sec.5 generates indeed the renormalized mean fields equations.

\section{Mean fields and induced sources}

In this section, after specifying some of the conventions and the notations used in this work (in subsection 2.1), we derive the first set of equations in the hierarchy of DysonSchwinger equations for $N$-point functions, i.e., the equations of motion for the average gauge and fermionic fields (in subsection 2.2). These equations relate the mean fields to 2 and 3-point functions which are interpreted here as induced sources for the mean fields. Functional relations between these induced sources and the one-particle irreducible amplitudes are recalled in subsection 2.3. The relation between induced sources and 2point functions is further analysed in the last subsection. Most of the material presented in this section is standard, and the equations written are exact; the approximations to be made will be presented in the next section. 


\section{$2.1 \quad$ Generalities}

We consider an ultrarelativistic plasma in, or close to, thermal equilibrum, at a temperature $T=1 / \beta$. The equations of motion which describe its long wavelength excitations will be derived using the imaginary time formalism, and their real time versions will be obtained through an analytic continuation. Since the only quantities to be continued in the present work are at most 2-point functions, the procedure is non ambiguous. It allows us to avoid the intricacies of real time formalisms and to preserve, at least in the first stage of our approximation scheme, the familiar structure of perturbation theory for systems in equilibrium. In doing the analytic continuation, the relevant 2-point functions may be chosen to obey retarded boundary conditions, appropriate to the calculation of the causal response functions that one is eventually interested in. We use Minkovski metric and we note $x=\left(x_{0}, \vec{x}\right)=(-i \tau, \vec{x}), \partial_{0}=i \partial_{\tau}$ and $\partial^{2}=-\partial_{\tau}^{2}-\vec{\nabla}^{2}$. In the analytic continuation, $x_{0}$ becomes a complex variable ( $\tau$ acquires an imaginary part) and the metric, the gamma matrices, etc..., are untouched. Our units are such that $\hbar=c=1$. We consider a $\mathrm{SU}(N)$ gauge theory with $N_{f}$ flavors of quarks . The color indices for the adjoint representation, $a, b, \ldots$ run from 1 to $N^{2}-1$, while those for the fundamental representation, $i, j, \ldots$ run from 1 to $N$. We work in four space-time dimensions, $d=4$, but on occasion we shall keep $d$ as an explicit parameter in our equations in order to exhibit the contribution of the various gauge degrees of freedom. Minkovski indices $\mu, \nu, \ldots$ run from 0 to $d-1$. The generators of the gauge group in different representations are taken to be Hermitian and traceless. They are denoted by $t^{a}$ and $T^{a}$, respectively, for the fundamental and the adjoint representations, and are normalized such that

$$
\operatorname{tr}\left(t^{a} t^{b}\right)=\frac{1}{2} \delta^{a b}, \quad \operatorname{tr}\left(T^{a} T^{b}\right)=N \delta^{a b} .
$$

Furthermore, $\left(T^{a}\right)_{b c}=-i f^{a b c}$, and $t^{a} t^{a}=C_{f}$, where $C_{f}=\left(N^{2}-1\right) /(2 N)$ is the Casimir of the fundamental representation and $f^{a b c}$ are the structure constants of the group:

$$
\left[t^{a}, t^{b}\right]=i f^{a b c} t^{c}
$$

We use, without distinction, upper and lower positions for the color indices.

The thermal averages of fields operators in the presence of external sources can be obtained from the generating functional

$$
\begin{aligned}
Z\left[j_{\mu}^{a}, \eta, \bar{\eta}, C^{a}, \bar{C}^{a}\right] & =\int\left[d \bar{\psi} d \psi d A_{\mu}^{a} d \bar{\zeta}^{b} d \zeta^{c}\right] \\
& \times \exp -\{S+(j, A)+(\bar{\eta}, \psi)+(\bar{\psi}, \eta)+(\bar{C}, \zeta)+(\bar{\zeta}, C)\}
\end{aligned}
$$


where $S$ is the usual QCD action in a covariant gauge

$$
S=\int_{0}^{\beta} d \tau d^{3} x\left\{\frac{1}{4} F_{\mu \nu}^{a} F^{\mu \nu a}+\frac{\lambda}{2}\left(\partial \cdot A^{a}\right)^{2}+\bar{\psi}(-i \not D) \psi+\bar{\zeta}^{a} \partial_{\mu}\left(\tilde{D}^{\mu} \zeta\right)^{a}\right\},
$$

and $\psi, \bar{\psi}, A, \zeta$ and $\bar{\zeta}$ denote, respectively, the quark fields, the gauge fields and the FadeevPopov ghosts. They obey antiperiodic (for $\psi, \bar{\psi}$ ) and periodic (for $A, \zeta$ and $\bar{\zeta}$ ) boundary conditions, with period $\beta$. In the formula above, $\lambda$ is the gauge fixing parameter, and $\not D \equiv \gamma^{\mu} D_{\mu}$, with the usual notations for the covariant derivatives in the fundamental $\left(D_{\mu}\right)$ and in the adjoint representations $\left(\tilde{D}_{\mu}\right)$ :

$$
D_{\mu} \equiv \partial_{\mu}+i g A_{\mu}(x), \quad \tilde{D}_{\mu} \equiv \partial_{\mu}+i g \tilde{A}_{\mu}(x)
$$

Here $A_{\mu} \equiv A_{\mu}^{a} t^{a}$ and $\tilde{A}_{\mu} \equiv A_{\mu}^{a} T^{a}$ are the gauge field matrices in the two representations. The source terms in eq. (2.3) are

$$
(j, A) \equiv \int_{0}^{\beta} d \tau d^{3} x j_{\mu}^{a}(x) A_{a}^{\mu}(x),
$$

and similarly for $(\bar{\eta}, \psi),(\bar{\psi}, \eta),(\bar{C}, \zeta)$ and $(\bar{\zeta}, C)$.

The gauge field strength tensor is $F_{\mu \nu} \equiv\left[D_{\mu}, D_{\nu}\right] /(i g) \equiv F_{\mu \nu}^{a} t^{a}$. For any operator of the form $O(x)=O^{a}(x) t^{a}$, we have

$$
\left[D_{\mu}, O(x)\right] \equiv \partial_{\mu} O(x)+i g\left[A_{\mu}(x), O(x)\right]
$$

and similarly in the adjoint representation.

\subsection{Equations of motion for the mean fields}

In the absence of the external sources, the plasma is in thermal equilibrium and the expectation values of the fields vanish. For nonvanishing sources, the fields acquire nontrivial expectation values which obey equations of motion that are readily derived from eq. (2.3):

$$
\begin{gathered}
\left\langle\left[D^{\nu}, F_{\nu \mu}(x)\right]\right\rangle+\lambda \partial_{\mu} \partial_{\nu}\left\langle A^{\nu}(x)\right\rangle-g t^{a}\left\langle\bar{\psi}(x) \gamma_{\mu} t^{a} \psi(x)\right\rangle+g t^{a} f_{a b c}\left\langle\left(\partial_{\mu} \bar{\zeta}^{b}(x)\right) \zeta^{c}(x)\right\rangle=j_{\mu}(x), \\
i \not \partial\langle\psi(x)\rangle-g\langle A(x) \psi(x)\rangle=\eta(x) \\
-\partial^{2}\left\langle\zeta^{a}(x)\right\rangle+g f^{a b c}\left\langle\partial^{\mu}\left(A_{\mu}^{b}(x) \zeta^{c}(x)\right)\right\rangle=C^{a}(x)
\end{gathered}
$$




$$
-\partial^{2}\left\langle\bar{\zeta}^{a}(x)\right\rangle+g f^{a b c}\left\langle A_{\mu}^{b}(x) \partial^{\mu} \bar{\zeta}^{c}(x)\right\rangle=\bar{C}^{a}(x),
$$

together with the Hermitian conjugate equation for $\langle\bar{\psi}\rangle$. The explicit form of the commutator term in eq. (2.8) is

$$
\left\langle\left[D^{\nu}, F_{\nu \mu}\right]^{a}\right\rangle=\left(g_{\mu \nu} \partial^{2}-\partial_{\mu} \partial_{\nu}\right)\left\langle A_{a}^{\nu}\right\rangle-g f^{a b c} \Gamma_{\mu \rho \lambda \nu}\left\langle A_{b}^{\nu}\left(\partial^{\lambda} A_{c}^{\rho}\right)\right\rangle-g^{2} f^{a b c} f^{c d e}\left\langle A_{\nu}^{b} A_{\mu}^{d} A_{e}^{\nu}\right\rangle,
$$

where

$$
\Gamma_{\mu \nu \rho \lambda} \equiv 2 g_{\mu \nu} g_{\rho \lambda}-g_{\mu \rho} g_{\nu \lambda}-g_{\mu \lambda} g_{\nu \rho} .
$$

Note the following identities which will be used later:

$$
\begin{gathered}
k^{\mu} \Gamma_{\mu \nu \rho \lambda} k^{\lambda}=k_{\nu} k_{\rho}-g_{\nu \rho} k^{2}, \\
\Gamma_{\mu \nu \rho \lambda}=\Gamma_{\nu \mu \rho \lambda}=\Gamma_{\mu \nu \lambda \rho}=\Gamma_{\rho \lambda \mu \nu}, \quad \Gamma_{\mu \nu \rho \lambda}+\Gamma_{\mu \rho \lambda \nu}+\Gamma_{\mu \lambda \nu \rho}=0 .
\end{gathered}
$$

We shall also use the following shorthand notation for the four gluon vertex:

$$
\begin{aligned}
\hat{\Gamma}_{\mu \nu \lambda \rho}^{a b c d} \equiv \frac{1}{2}\left\{f^{e a b} f^{e c d}\left(g_{\mu \lambda} g_{\nu \rho}-g_{\mu \rho} g_{\nu \lambda}\right)\right. & +f^{e a c} f^{e d b}\left(g_{\mu \rho} g_{\lambda \nu}-g_{\mu \nu} g_{\lambda \rho}\right) \\
& \left.+f^{e a d} f^{e b c}\left(g_{\mu \nu} g_{\rho \lambda}-g_{\mu \lambda} g_{\rho \nu}\right)\right\} .
\end{aligned}
$$

Note that $\hat{\Gamma}_{\mu \nu \rho \lambda}^{a b c d}$ and $\Gamma_{\mu \nu \rho \lambda}$ are related by

$$
\hat{\Gamma}_{\mu \nu \rho \lambda}^{a b c d} A_{c}^{\rho} A_{d}^{\lambda} \equiv-f^{a d e} f^{b c e} \Gamma_{\mu \rho \nu \lambda} A_{c}^{\rho} A_{d}^{\lambda} .
$$

We separate the 2-point functions into connected parts, denoted by the symbol $\langle\cdots\rangle_{c}$, and disconnected parts. For example, $\langle A(x) \psi(x)\rangle=\langle A(x) \psi(x)\rangle_{c}+\langle A(x)\rangle\langle\psi(x)\rangle$, and $\left\langle\bar{\psi} \gamma_{\mu} t^{a} \psi\right\rangle=\left\langle\bar{\psi} \gamma_{\mu} t^{a} \psi\right\rangle_{c}+\langle\bar{\psi}\rangle \gamma_{\mu} t^{a}\langle\psi\rangle$. Then eq. (2.12) can be written compactly as follows:

$$
\begin{aligned}
\left\langle\left[D^{\nu}, F_{\nu \mu}\right]^{a}\right\rangle & =\left[\mathcal{D}^{\nu}, \mathcal{F}_{\nu \mu}\right]^{a}-g f^{a b c} \Gamma_{\mu \rho \lambda \nu}\left\langle A_{b}^{\nu}\left(\partial^{\lambda} A_{c}^{\rho}\right)\right\rangle_{c} \\
& -g^{2} f^{a b c} f^{c d e}\left\langle A_{\nu}^{b} A_{\mu}^{d} A_{e}^{\nu}\right\rangle_{c}-g^{2} \hat{\Gamma}_{\mu \nu \rho \lambda}^{a b c d}\left\langle A_{b}^{\nu}\right\rangle\left\langle A_{c}^{\rho} A_{d}^{\lambda}\right\rangle_{c},
\end{aligned}
$$

where $\mathcal{D}_{\mu} \equiv \partial_{\mu}+i g\left\langle A_{\mu}(x)\right\rangle$ and $\mathcal{F}_{\mu \nu} \equiv\left[\mathcal{D}_{\mu}, \mathcal{D}_{\nu}\right] /(i g)$.

After the separation of the connected parts in the 2 and 3-point functions, the mean field equations (2.8)-(2.11) can be written as:

$$
\begin{gathered}
{\left[\mathcal{D}^{\nu}, \mathcal{F}_{\nu \mu}(x)\right]^{a}+\lambda \partial_{\mu} \partial_{\nu}\left\langle A_{a}^{\nu}(x)\right\rangle-g\langle\bar{\psi}(x)\rangle \gamma_{\mu} t^{a}\langle\psi(x)\rangle+g f^{a b c}\left(\partial_{\mu}\left\langle\bar{\zeta}^{b}(x)\right\rangle\right)\left\langle\zeta^{c}(x)\right\rangle} \\
=j_{\mu}^{a}(x)+j_{\mu}^{\text {inda }}(x),
\end{gathered}
$$




$$
\begin{gathered}
(i \not \partial-g\langle A(x)\rangle)\langle\psi(x)\rangle=\eta(x)+\eta^{i n d}(x), \\
-\partial^{2}\left\langle\zeta^{a}(x)\right\rangle+g f^{a b c} \partial^{\mu}\left(\left\langle A_{\mu}^{b}(x)\right\rangle\left\langle\zeta^{c}(x)\right\rangle\right)=C^{a}(x)+C^{\text {ind } a}(x), \\
-\partial^{2}\left\langle\bar{\zeta}^{a}(x)\right\rangle+g f^{a b c}\left\langle A_{\mu}^{b}(x)\right\rangle \partial^{\mu}\left\langle\bar{\zeta}^{c}(x)\right\rangle=\bar{C}^{a}(x)+\bar{C}^{\text {ind } a}(x) .
\end{gathered}
$$

In the left hand sides of these equations we have isolated the terms corresponding to the tree level equations of motion generated by the QCD action (2.4). In their right hand sides, we have, next to the external sources, connected 2 and 3-point Green's functions which we refer as "induced sources", because they play a role similar to that of $\eta, j_{\mu}$, or $C$, that is, they act as extra sources for the mean fields. Explicitly, they are

$$
\begin{gathered}
j_{\mu}^{i n d a}(x) \equiv g\left\langle\bar{\psi} \gamma_{\mu} t^{a} \psi\right\rangle_{c}-g f^{a b c}\left\langle\left(\partial_{\mu} \bar{\zeta}^{b}\right) \zeta^{c}\right\rangle_{c}+g f^{a b c} \Gamma_{\mu \rho \lambda \nu}\left\langle A_{b}^{\nu}\left(\partial^{\lambda} A_{c}^{\rho}\right)\right\rangle_{c} \\
+g^{2} \hat{\Gamma}_{\mu \nu \rho \lambda}^{a b c d}\left\langle A_{b}^{\nu}\right\rangle\left\langle A_{c}^{\rho} A_{d}^{\lambda}\right\rangle_{c}+g^{2} f^{a b c} f^{c d e}\left\langle A_{\nu}^{b} A_{\mu}^{d} A_{e}^{\nu}\right\rangle_{c}, \\
\eta^{i n d}(x) \equiv g \gamma^{\nu} t^{a}\left\langle A_{\nu}^{a}(x) \psi(x)\right\rangle_{c}, \\
C^{a i n d}(x) \equiv-g f^{a b c}\left\langle\partial^{\mu}\left(A_{\mu}^{b}(x) \zeta^{c}(x)\right)\right\rangle_{c}, \\
\bar{C}^{a i n d}(x) \equiv-g f^{a b c}\left\langle A_{\mu}^{b}(x) \partial^{\mu} \bar{\zeta}^{c}(x)\right\rangle_{c} .
\end{gathered}
$$

In equilibrium, when the external sources vanish, the induced sources are also vanishing. This follows from symmetry. In equilibrium, the expectation values involve thermal averages over color singlet states, and elementary group theory can then be used to prove that, in this case, all terms on the r.h.s. of eqs. (2.22)-(2.25) indeed vanish.

At this point, it is perhaps useful to illustrate the structure of the equations of motion by means of diagrams. This is done in Figs.1 and 2, which represent schematically the various contributions to the expectation values of the fields, as obtained from the equations of motion (2.18) and (2.19).

\subsection{Induced sources and one particle irreducible amplitudes}

It will be convenient later to express the induced sources as functionals of the mean fields. Following a standard procedure, this may be achieved in a systematic fashion by introducing the effective action, that is, the Legendre transform of $\ln Z$, with $Z$ given 
by eq. (2.3). The external sources are then considered as the first derivatives of the effective action with respect to the mean fields, which are treated as independent variables. Further derivations yield the one particle irreducible amplitudes. Thus, by differentiating eqs. (2.18) and (2.19) with respect to the mean fields, one gets, using the usual definitions of self energies,

$$
\begin{array}{cc}
-\frac{\delta j_{\mu}^{a}(x)}{\delta\left\langle A_{b}^{\nu}(y)\right\rangle}=\delta^{a b} D_{0 \mu \nu}^{-1}+\Pi_{\mu \nu}^{a b}(x, y), & \Pi_{\mu \nu}^{a b}(x, y)=\frac{\delta j_{\mu}^{i n d a}(x)}{\delta\left\langle A_{b}^{\nu}(y)\right\rangle} \\
-\frac{\delta \eta(x)}{\delta\langle\psi(y)\rangle}=(-i \not \partial+g\langle A(x)\rangle) \delta(x-y)+\Sigma(x, y), & \Sigma(x, y)=\frac{\delta \eta^{i n d}(x)}{\delta\langle\psi(y)\rangle} .
\end{array}
$$

In eq. $(2.26)$

$$
D_{0 \mu \nu}^{-1}(x, y) \equiv\left(-g_{\mu \nu} \partial^{2}+(1-\lambda) \partial_{\mu} \partial_{\nu}\right) \delta(x-y)
$$

is the free inverse propagator for gluons. A further differentiation gives the 3-point functions. The quark-gluon vertex is

$$
g \gamma^{\mu} t^{a}+g \Gamma_{a}^{\mu}(x, y, z)=-\frac{\delta^{2} j_{a}^{\mu}(x)}{\delta\langle\psi(z)\rangle \delta\langle\bar{\psi}(y)\rangle}=-\frac{\delta^{2} \eta(y)}{\delta\left\langle A_{\mu}^{a}(x)\right\rangle \delta\langle\psi(z)\rangle}
$$

that is,

$$
g \Gamma_{\mu}^{a}(x, y, z)=\frac{\delta \Sigma(y, z)}{\delta\left\langle A_{a}^{\mu}(x)\right\rangle}=\frac{\delta^{2} j_{\mu}^{i n d a}(x)}{\delta\langle\psi(z)\rangle \delta\langle\bar{\psi}(y)\rangle} .
$$

Similarly, we get for the vertex correction to the three-gluons amplitude:

$$
g \Gamma_{\mu \nu \rho}^{a b c}(x, y, z)=\frac{\delta^{2} j_{\mu}^{i n d a}(x)}{\delta\left\langle A_{b}^{\nu}(y)\right\rangle \delta\left\langle A_{c}^{\rho}(z)\right\rangle}=\frac{\delta \prod_{\mu \nu}^{a b}(x, y)}{\delta\left\langle A_{c}^{\rho}(z)\right\rangle} .
$$

We do not write here the expressions corresponding to amplitudes with ghost external lines since we shall not need them.

\subsection{Induced sources and two point functions}

By inspecting eqs. (2.22)-(2.25), one sees that the induced sources are given by offequilibrum 2-point functions. The only exception is the induced current $j{ }^{\text {ind }}$ which also contains the 3-point function $\left\langle A_{\nu}^{b} A_{\mu}^{d} A_{e}^{\nu}\right\rangle_{c}$. However, in the present work, we shall be interested only in the contributions to the induced sources which appear at leading order in $g$. Since the connected 3-point function contains at least two powers of $g$ more than 
the other terms, it can be safely ignored. This allows us to express the induced current $j^{\text {ind }}(2.22)$ in terms of 2-point functions only. We write

$$
j_{\mu}^{i n d a}(x) \equiv j_{\mathrm{f} \mu}^{a}(x)+j_{\mathrm{G} \mu}^{a}(x)+j_{\mathrm{g} \mu}^{a}(x)+j_{\Omega \mu}^{a}(x),
$$

where the subscripts ( $\mathrm{f}, \mathrm{G}, \mathrm{g}, \Omega$ ) refer to which hard particles contribute to the induced current. Thus $j_{\mathrm{f}}$ is the current associated with the polarization of the hard fermions induced by the average quark and gauge fields

$$
j_{\mathrm{f} \mu}^{a}(x) \equiv g\left\langle\bar{\psi}(x) \gamma_{\mu} t^{a} \psi(x)\right\rangle_{c} .
$$

Similarly, $j_{\mathrm{G}}$ results from the polarization of the hard ghost particles induced by the gauge mean fields

$$
j_{G \mu}^{a}(x) \equiv-g f^{a b c}\left\langle\left(\partial_{\mu} \bar{\zeta}^{b}(x)\right) \zeta^{c}(x)\right\rangle_{c} .
$$

The role of this current is to eliminate spurious polarization effects which, in a covariant gauge, are due to non-physical gluonic degrees of freedom. The induced current resulting from the polarization of the hard gluons by the quark and gauge mean fields is

$$
j_{\mathrm{g} \mu}^{a}(x) \equiv g f^{a b c} \Gamma_{\mu \rho \lambda \nu}\left\langle A_{b}^{\nu}(x)\left(\partial^{\lambda} A_{c}^{\rho}(x)\right)\right\rangle_{c} .
$$

The last term in eq. (2.32) is the "tadpole" contribution

$$
j_{\Omega \mu}^{a}(x) \equiv g^{2} \hat{\Gamma}_{\mu \nu \rho \lambda}^{a b c d}\left\langle A_{b}^{\nu}(x)\right\rangle\left\langle A_{c}^{\rho}(x) A_{d}^{\lambda}(x)\right\rangle_{c} .
$$

It is convenient to express the induced sources in terms of time-ordered propagators, for which we shall derive equations of motion in the next section. Let us first introduce specific notations. Aside from the usual quark, gluon and ghost propagators,

$$
\begin{aligned}
S(x, y) & \equiv\langle\mathrm{T} \psi(x) \bar{\psi}(y)\rangle_{c}=-\frac{\delta\langle\psi(x)\rangle}{\delta \eta(y)}, \\
D_{\mu \nu}^{a b}(x, y) & \equiv\left\langle\mathrm{T} A_{\mu}^{a}(x) A_{\nu}^{b}(y)\right\rangle_{c}=-\frac{\delta\left\langle A_{\mu}^{a}(x)\right\rangle}{\delta j_{b}^{\nu}(y)}, \\
G^{a b}(x, y) & \equiv\left\langle\mathrm{T}^{a}(x) \bar{\zeta}^{b}(y)\right\rangle_{c}=-\frac{\delta\left\langle\zeta^{a}(x)\right\rangle}{\delta C^{b}(y)},
\end{aligned}
$$

we shall also need the following "abnormal" propagators

$$
\begin{aligned}
K_{\nu}^{a}(x, y) & \equiv\left\langle\mathrm{T} \psi(x) A_{\nu}^{a}(y)\right\rangle_{c}=-\frac{\delta\langle\psi(x)\rangle}{\delta j_{a}^{\nu}(y)}=-\frac{\delta\left\langle A_{\nu}^{a}(y)\right\rangle}{\delta \bar{\eta}(x)} \\
H_{\nu}^{a}(x, y) & \equiv\left\langle\mathrm{T} A_{\nu}^{a}(x) \bar{\psi}(y)\right\rangle_{c}=-\frac{\delta\langle\bar{\psi}(y)\rangle}{\delta j_{a}^{\nu}(x)}=\frac{\delta\left\langle A_{\nu}^{a}(x)\right\rangle}{\delta \eta(y)} \\
O_{\nu}^{a b}(x, y) & \equiv\left\langle\mathrm{T} \zeta^{a}(x) A_{\nu}^{b}(y)\right\rangle_{c}=-\frac{\delta\left\langle\zeta^{a}(x)\right\rangle}{\delta j_{b}^{\nu}(y)}=-\frac{\delta\left\langle A_{\nu}^{b}(y)\right\rangle}{\delta \bar{C}^{a}(x)} \\
\bar{O}_{\nu}^{a b}(x, y) & \equiv\left\langle\mathrm{T} A_{\nu}^{a}(x) \bar{\zeta}^{b}(y)\right\rangle_{c}=-\frac{\delta\left\langle\bar{\zeta}^{b}(y)\right\rangle}{\delta j_{a}^{\nu}(x)}=\frac{\delta\left\langle A_{\nu}^{a}(x)\right\rangle}{\delta C^{b}(y)}
\end{aligned}
$$


which, of course, vanish in equilibrium. In these definitions, the symbol $\mathrm{T}$ denotes time ordering with respect to imaginary time. The identities listed in the right hand sides of the previous expressions will be useful later, when we derive the equations of motion for 2-point functions (see Sec.3.3).

The time ordered propagators can be separated into components which are analytic functions of their time arguments [31, 32]. For example, for the fermion propagator, we write

$$
S(x, y) \equiv \theta\left(\tau_{x}-\tau_{y}\right) S^{>}(x, y)-\theta\left(\tau_{y}-\tau_{x}\right) S^{<}(x, y)
$$

with similar definitions for the other 2-point functions $D, G, K_{\nu}^{a}, H_{\nu}^{a}, O_{\nu}^{a b}$ and $\bar{O}_{\nu}^{a b}$, but without the minus sign (remember that the ghosts fields, while anticommuting, are submitted to periodic boundary conditions in imaginary time). The two functions $S^{>}(x, y)$ and $S^{<}(x, y)$ are analytic functions of $x_{0}$ and $y_{0}$ for $-\beta<\operatorname{Im}\left(x_{0}-y_{0}\right)<0$ and $0<$ $\operatorname{Im}\left(x_{0}-y_{0}\right)<\beta$ respectively. This is the case at equilibrium; we assume that the sources satisfy the the conditions necessary to preserve this property off-equilibrium[31]. We may assume, to be specific, that the (analytic) sources are adiabatically switched on at (real) time $-\infty$. Then, when (real) time goes to $-\infty$, the various 2 -point functions go over to their equilibrium values in the absence of sources, and, for arbitrary time, they represent correlation functions averaged over the initial equilibrium grand canonical ensemble.

The induced sources eqs. (2.23)-(2.25) and (2.33)-(2.36) involve products of fields with equal time arguments $\tau_{x}=\tau_{y}$. They may be calculated from the time ordered propagators (2.37)-(2.38) taking consistently $\tau_{y}-\tau_{x}=\eta \rightarrow 0^{+}$. Alternatively, they may be expressed in terms of the analytic pieces of these propagators, as defined in (2.39):

$$
\begin{gathered}
\eta^{i n d}(x)=g \gamma^{\nu} t^{a} K_{a \nu}^{<}(x, x), \\
j_{\mathrm{f} \mu}^{a}(x)=g \operatorname{Tr}\left(\gamma_{\mu} t^{a} S^{<}(x, x)\right),
\end{gathered}
$$

where the trace acts both on spinor and color indices,

$$
\begin{gathered}
j_{\mathrm{G} \mu}^{a}(x)=-\left.g f^{a b c}\left[\partial_{\mu}^{y} G_{b c}^{<}(x, y)\right]\right|_{y \rightarrow x^{+}}, \\
j_{\mathrm{g} \mu}^{a}(x)=\left.g f^{a b c} \Gamma_{\mu \rho \lambda \nu}\left[\partial_{x}^{\lambda} D_{c b}^{\rho \nu<}(x, y)\right]\right|_{y \rightarrow x^{+}}, \\
C_{a}^{i n d}(x)=\left.g f^{a b c}\left[\left(\partial_{y}^{\mu}+\partial_{x}^{\mu}\right) O_{\mu}^{b c<}(x, y)\right]\right|_{y \rightarrow x^{+}},
\end{gathered}
$$




$$
\bar{C}_{a}^{i n d}(x)=-\left.g f^{a b c}\left[\partial_{y}^{\mu} \bar{O}_{\mu}^{b c<}(x, y)\right]\right|_{y \rightarrow x^{+}},
$$

where $y \rightarrow x^{+}$stands for $\tau_{y}-\tau_{x} \rightarrow 0^{+}$. These expressions hold after analytic continuation to real time. Then $y \rightarrow x^{+}$should be understood as $y_{0}-x_{0}=i \eta$ and $\eta \rightarrow 0^{+}$.

Because the induced sources involve products of fields at the same point, one could expect to encounter ultraviolet divergences when calculating them. However, this will not be the case here (with the exception of the tadpole term to be discussed in the next section). Indeed, as we have seen, in equilibrum all the induced sources vanish, for symmetry reasons. Off-equilibrum, no divergence occurs at leading order, as we shall verify later.

For further reference, we list here expressions for analytic components of the free, equilibrum propagators. For the fermions, we have

$$
S_{0}^{<}(x, y)=\int \frac{d^{4} k}{(2 \pi)^{4}} e^{-i k \cdot(x-y)} S_{0}^{<}(k), \quad S_{0}^{<}(k)=\not k \rho_{0}(k) n\left(k_{0}\right) \equiv \not k \tilde{\Delta}(k),
$$

where $\rho_{0}(k)$ is the spectral function

$$
\rho_{0}(k)=2 \pi \epsilon\left(k_{0}\right) \delta\left(k^{2}\right)
$$

$n\left(k_{0}\right)=1 /\left(\exp \left(\beta k_{0}\right)+1\right)$ is the fermion occupation factor, and $\epsilon\left(k_{0}\right)=k_{0} /\left|k_{0}\right|$. Similarly the equilibrium free gluon 2-point function $D_{0 \mu \nu}^{<}(k)$ is, in Feynman gauge $(\lambda=1)$,

$$
D_{0 \mu \nu}^{<a b}(k)=-\delta^{a b} g_{\mu \nu} \Delta(k)
$$

where now $\Delta(k) \equiv \rho_{0}(k) N\left(k_{0}\right)$, and $N\left(k_{0}\right)=1 /\left(\exp \left(\beta k_{0}\right)-1\right)$ denotes the boson occupation factor. Finally, for the free ghost propagator, we have

$$
G_{0 a b}^{<}(k)=\delta^{a b} \Delta(k),
$$

because of the periodic boundary conditions imposed on the ghost fields. We note that in the description of the plasma using a covariant gauge, the equilibrum state contains ghost particles obeying Bose statistics. These are unphysical degrees of freedom, whose role is to cancel, when computing physical observables, the contribution of the longitudinal gluons, which are also present in the state, according to the propagator (2.48). 


\section{Equations for the induced sources}

Up to now all the equations that we have written are exact, but incomplete. Indeed, these are equations of motion for the mean fields which depend on induced sources given themselves by 2-point functions. (A possible contribution from a 3-point function has been seen to be negligible in the previous section). These 2-point functions are determined by equations which involve generally 3 and 4 -point functions, and so on. The approximations to be presented in this section will provide us with equations for the 2-point functions which involve the mean fields, but no higher point functions, thereby closing the hierarchy.

The various features of our approximation scheme are summarized in subsection 3.1. Then, in the following subsections, we go on and derive systematically the equations of motion for the various 2-point functions of interest. This is done in several steps. In subsection 3.3 we take into account the smallness of the coupling in order to truncate the hierarchy. In the next subsections, we implement, for each 2-point function of interest, the approximations which follow from the fact that both the wavenumber and the amplitude of the mean field oscillations are also small. The technical problems arising from the use of a general covariant gauge are discussed in Appendices A and B.

\subsection{Approximation scheme}

The cornerstone of our approximation scheme is the fact that the long wavelength excitations that we are studying are collective excitations which develop at a particular energy scale, $g T$, well separated, when $g \ll 1$, from the typical energies of plasma particles, $\sim T$. This scale controls both the wavelength and the amplitude of the mean field oscillations. Collectivity arises because the wavelength of the oscillations, $\sim 1 / g T$, is much larger than the typical interparticle distance, $\sim 1 / T$.

The way how the scale $g T$ emerges, is perhaps best seen on the equations (2.18)(2.21) for the mean fields. Technically speaking, one expects collective excitations to develop whenever the induced sources on the right hand sides of these equations are of the same order of magnitude as the first order differential operators which stand on their l.h.s. Assuming that the wavelength/period of the field oscillations is $\lambda$ (i.e., the fields carry a typical momentum $P \sim g T$ ), the l.h.s. are then of order $\sim \lambda^{-1} \phi$, where $\phi$ denotes one of the average fields, that is either $\left\langle F_{\mu \nu}\right\rangle$ or $\langle\psi\rangle$. The r.h.s., that is, the induced sources are indeed of order $\lambda^{-1} \phi$ when $\lambda^{-1}$ is of order $g T$. For instance, eq. (2.27) shows that $\eta^{\text {ind }} \sim \Sigma\langle\psi\rangle$. The dominant contribution to $\Sigma(P)$ at high temperature is $\sim g^{2} T^{2} / P$. Thus, $\eta^{\text {ind }} \sim\left(g^{2} T^{2} / P\right)\langle\psi\rangle$, which is $\sim P\langle\psi\rangle \sim \lambda^{-1}\langle\psi\rangle$ when $P \sim g T$. 
Similarly, $j^{\text {ind }} \sim \Pi\langle A\rangle \sim g^{2} T^{2}\langle A\rangle$ at high temperature, while $\partial\langle F\rangle \sim \partial^{2}\langle A\rangle \sim P^{2}\langle A\rangle$; again, $j^{\text {ind }} \sim \partial\langle F\rangle$ if $P \sim g T$. Furthermore, the requirement that the plasma stay close to equilibrium puts constraints on the strength of the mean fields which also depend on $\lambda$. The existence of such constraints can be anticipated on the basis of the following heuristic argument. The plasma will be weakly perturbed if, for instance, the color force $\sim g\langle F\rangle$ acting on a typical particle with momentum $k \sim T$, produces a small change in its momentum. Assuming that the force is acting during a time $\Delta t \sim \lambda$, we require $\Delta k=g\langle F\rangle \lambda \ll k$. One sees that, for $\lambda^{-1} \sim g T$ and $\Delta k \lesssim g T$, we have $\langle F\rangle \lesssim g T^{2}$. More generally, for $\lambda^{-1} \sim g T$, there exists a limit in which all terms in the equations of motion are of the same order of magnitude. This is achieved when $\langle F\rangle \sim g T^{2}$ and $\langle\bar{\psi}\rangle\langle\psi\rangle \sim g T^{3}$, that is, when these fields are $g$ times a power of $T$ equal to their canonical dimension. When the fields take this particular limiting strength, the coupling constant disappears from the equations of motion if one expresses all energies and momenta in units of $g T$, and length and time in units of $1 / g T$. Furthermore, in this limit, all terms in the field strength tensor, or in covariant derivatives, are of the same order of magnitude ( $\left.\partial \sim g\langle A\rangle, \partial\langle A\rangle \sim g\langle A\rangle^{2}\right)$, and the same is true for all the contributions to the induced sources which are non linear in $\langle A\rangle$. In the rest of this work, we shall work in this limit, where the equations keep their full non-abelian structure. However, one should emphasize that the equations to be derived are also valid in the case of weaker fields. In the limit of weak fields, the equations for $\langle A\rangle$ and $\langle\psi\rangle$ decouple, and $\langle A\rangle$ behaves as an abelian gauge field, that is, the equations are linear.

We turn now to the 2-point functions which are needed for the calculation of the induced sources and explain the general structure of our approximation scheme. For the present discussion, we shall consider as a generic example the off-equilibrium fermion 2-point function $S(x, y)$. Most arguments extend to other 2-point functions as well.

The equations of motion for the 2-point functions will be obtained in Sec.3.3, by differentiation of the mean field equations with respect to the external sources. In doing this, we make use of the identities listed in eqs. (2.37)-(2.38). For $S(x, y)$ one obtains (in the case $\langle\psi\rangle=\langle\bar{\psi}\rangle=0$ )

$$
\left(S_{0}^{-1}+g\langle A\rangle+\Sigma\right) S=1,
$$

where operator notations have been used, and $S_{0}^{-1}(x, y) \equiv-i \not \phi_{x} \delta(x-y)$. The offequilibrium self-energy $\Sigma$ arises from differentiating the induced source. We argue now that such a term can, in fact, be ignored in leading order. To understand why it is so, let us recall that we need $S(x, y)$ only to evaluate the induced current $j_{\mathrm{f}}$, according to eq. (2.41). Since the equilibrium propagator $S_{e q}(x-y)$ does not contribute to $j_{\mathrm{f}}$, we need, 
in fact, only the deviation induced by the gauge fields, $\delta S \equiv S-S_{e q}$. Furthermore, as will be clear shortly, we only need $\delta S(x, y)$ for small relative distances $|x-y| \lesssim 1 / T$, or, equivalently, for hard momenta $k \sim T$. The diagrams contributing to $\delta S$ involve at least one mean field insertion, and the lowest order ones are shown in Figs.3a and 3b. An elementary analysis reveals that, indeed, those in Fig.3b, which represent typical corrections coming from $\Sigma$, are negligible compared to that of Fig.3a when $k \sim T$. Consequently, we shall neglect $\Sigma$ in eq. (3.1) and, at the same time, replace $S_{e q}$ by its free value $S_{0}$. We may then write the final equation as

$$
\left(S_{0}^{-1}+g\langle A\rangle\right) S=1, \quad S=S_{0}+\delta S
$$

More generally, in deriving the equations of motion for the 2-point functions in Sec.3.2, we shall ignore all terms coming from differentiating the induced sources, to which we refer as radiative corrections, and at same time replace the equilibrium propagators by their free expressions. This approximation, equivalent to the familiar one-loop approximation to the induced currents (see Figs.7 and 8 below), closes the hierarchy of equations at the level of 2-point functions. The net result is that only mean field insertions are to be considered in the off equilibrium 2-point functions. In physical terms, this is to say that the dominant interactions in the plasma are those between the hard particles and the mean fields.

It should be stressed that the one-loop approximation alone is not by itself a consistent approximation for the calculation of the induced sources. It does contain all leading terms in the weak coupling expansion, but also terms which are subleading, and which should therefore be discarded. These subleading contributions will be eliminated after taking carefully into account the other sources of $g$ dependence, namely, the soft character of the average fields.

In order to implement the approximation that the fields are slowly varying in space and time, we need to consider the 2-point functions in real time. As explained in section 1 , these functions, as well as the equations that they satisfy, can be obtained by analytic continuation of the imaginary time functions and the corresponding equations. We can then proceed to a gradient expansion, for which it is convenient to use the Wigner transforms of the various 2-point functions. In the case of the fermion propagator, this is

$$
\mathcal{S}(k, X) \equiv \int d^{4} s e^{i k \cdot s} S^{<}\left(X+\frac{s}{2}, X-\frac{s}{2}\right),
$$

where

$$
s^{\mu} \equiv x^{\mu}-y^{\mu}, \quad X^{\mu} \equiv \frac{x^{\mu}+y^{\mu}}{2} .
$$


Note that in order to avoid the proliferation of symbols, we use similar symbols to denote different functions. Thus, for example, we shall often write $S^{<}(s, X)$ in place of $S^{<}(X+$ $s / 2, X-s / 2)=S^{<}(x, y)$. Which function is meant can be recognized from its arguments. Wigner functions will be denoted consistently with calligraphic capitals. In equilibrium, the non vanishing two point functions are independent of $X^{\mu}$ and sharply peaked about $s^{\mu}=0$, the range of variation being fixed by the thermal wavelength $\lambda_{T} \sim 1 / T$. In the presence of a slowly varying disturbance, with a wavelength of order $1 / g T$, we expect the $s^{\mu}$ dependence to be little affected, while the acquired $X$ dependence will have a long wavelength variation. This will enable us, for example, to neglect the derivatives with respect to $X^{\mu}$, of order $g T$, relative to those with respect to $s^{\mu}$, of order $T$. We shall thus transform the equations of motion for the 2-point functions into equations for $\delta \mathcal{S}(k, X)$, valid for $k \sim T$ and $\partial_{X} \sim g T$.

One can use the Wigner function (3.3) to write the induced current (2.41) as

$$
j_{\mathrm{f} \mu}^{a}(X)=g \int \frac{d^{4} k}{(2 \pi)^{4}} \operatorname{Tr} \gamma_{\mu} t^{a} \delta \mathcal{S}(k, X),
$$

where $\delta \mathcal{S}(k, X) \equiv \mathcal{S}(k, X)-S_{e q}(k)$. This expression explains why we need $\delta \mathcal{S}(k, X)$ only for $k \sim T$ : soft momenta $k \sim g T$ represent a small integration region and, consequently, give generally a small contribution to such an integral. We are assuming that $\delta \mathcal{S}(k, X)$ is not infrared singular, which is the case in leading order. Note that $\delta \mathcal{S}(k, X)$ is not accurately determined by our approximate equation (3.2) if $k \sim g T$ (then the one loop diagrams in Fig.3b contribute at the same order as the tree-level diagram in Fig.3a). However, (3.2) provides a $\delta \mathcal{S}(k, X)$ with a smooth behaviour at small $k$ and the integration in (3.5) can be extended to all momenta without harm.

After solving the equations of motion for the 2-point functions, we find that, for fields as strong as allowed, the induced pieces of the propagators $\delta S, \delta D$ and $\delta G$, are, at most, $g$ times the corresponding equilibrium propagators. It is in this sense that the induced effects are perturbatively small, and that the plasma is weakly disturbed. When isolating the leading order terms in our equations, we are led to retain terms of lowest order in the fermionic fields, that is, terms linear in $\langle\psi\rangle$ and $\langle\bar{\psi}\rangle$ for $\mathcal{K}$ and $\mathcal{H}$, and, owing to baryon number conservation, bilinear expressions like $\langle\bar{\psi}\rangle\langle\psi\rangle$ for $\delta \mathcal{S}$ and $\delta \mathcal{D}$. However, in order to satisfy gauge covariance, we have to keep terms to all orders in $\langle A\rangle$. In fact, the gauge fields enter our equations in two different ways. This may be seen by rewriting eq. (3.1) as

$$
(-i \not \supset+g\langle A\rangle) \delta S=-g\langle A\rangle S_{0} .
$$

The gauge field enters linearly on the right hand side, as a usual perturbation. It also 
enters non linearly, as part of a covariant derivative, on the left hand side. The existence of these covariant derivatives, in the equations for both the mean fields and the 2-point functions, will be seen to be the only source of non linearity in $\langle A\rangle$. This particular way in which the mean fields enter our equations is the origin of the fact that there are hard thermal loops with any number of gluon lines, but no more than two fermion lines; and also of the fact that, in QED, there is no hard thermal loop with more than two photon external lines.

\subsection{The induced sources and their densities}

According to eq. (3.5), the induced current $j_{\mathrm{f} \mu}^{a}(X)$ can be written as an integral over $k$ of a density $\mathcal{J}_{\mathrm{f} \mu}^{a}(k, X)$ :

$$
j_{\mathrm{f} \mu}^{a}(X) \equiv g \int \frac{d^{4} k}{(2 \pi)^{4}} \mathcal{J}_{\mathrm{f} \mu}^{a}(k, X), \quad \mathcal{J}_{\mathrm{f} \mu}^{a}(k, X)=\operatorname{Tr} \gamma_{\mu} t^{a} \delta \mathcal{S}(k, X) .
$$

Once all 2-point functions are expressed in terms of Wigner functions, this can be generalized to all induced sources. In order to facilitate the reading of this paper, we now specify the systematics of the notation used for the various densities of induced sources. All these densities will be denoted by calligraphic capitals, as the Wigner functions to which they are related.

According to eq. (2.40), the induced source $\eta^{\text {ind }}(X)$ is given by

$$
\eta^{\text {ind }}(X)=g \int \frac{d^{4} k}{(2 \pi)^{4}} \not K(k, X)
$$

where $\mathbb{K}(k, X) \equiv t^{a} \gamma^{\mu} \mathcal{K}_{\mu}^{a}(k, X)$. The densities of the induced currents will carry, besides Minkowski and color indices, subscripts (f, g, $G, \Omega)$ and upperscripts $(A, \psi)$. As indicated after eq. (2.32), the subscripts refer to which hard particles are contributing to the induced sources, f standing for fermions, $g$ for gluons, $G$ for ghosts; $\Omega$ refers to the tadpole contribution. The upperscript indicates the nature of the background field which induces the current ( $A$ for a gauge field, and $\psi$ for a fermionic field). We shall see that, in the present approximation, one can separate the contributions of the fermionic and gauge fields, (up to trivial color "rotations" imposed by the gauge transformation properties of the various densities). Thus, we shall have a collection of induced currents $\left\{j_{\mathrm{f}}^{A}, j_{\mathrm{g}}^{A}, j_{G}^{A}, j_{\Omega}^{A}, j_{\mathrm{f}}^{\psi}, j_{\mathrm{g}}^{\psi}\right\}$, together with the corresponding densities $\left\{\mathcal{J}_{\mathrm{f}}^{A}, \mathcal{J}_{\mathrm{g}}^{A}, \mathcal{J}_{G}^{A}, \mathcal{J}_{\Omega}^{A}, \mathcal{J}_{\mathrm{f}}^{\psi}, \mathcal{J}_{\mathrm{g}}^{\psi}\right\}$. In some cases, it will be convenient to operate various grouping of these contributions. The absence of the upperscript will imply a grouping of all contributions corresponding to the various 
upperscripts:

$$
\mathcal{J}_{\mathrm{f}}=\mathcal{J}_{\mathrm{f}}^{A}+\mathcal{J}_{\mathrm{f}}^{\psi}, \quad \mathcal{J}_{\mathrm{g}}=\mathcal{J}_{\mathrm{g}}^{A}+\mathcal{J}_{\mathrm{g}}^{\psi}, \quad \mathcal{J}_{G}=\mathcal{J}_{G}^{A}, \quad \mathcal{J}_{\Omega}=\mathcal{J}_{\Omega}^{A}
$$

Similarly, the absence of a subscript implies a grouping of all contributions corresponding to the various possible subscripts:

$$
\mathcal{J}^{A}=\mathcal{J}_{\mathrm{f}}^{A}+\mathcal{J}_{\mathrm{g}}^{A}+\mathcal{J}_{G}^{A}+\mathcal{J}_{\Omega}^{A}, \quad \mathcal{J}^{\psi}=\mathcal{J}_{\mathrm{f}}^{\psi}+\mathcal{J}_{\mathrm{g}}^{\psi} .
$$

We shall also use the subscript b to group together all the contributions coming from bosonic particles:

$$
\mathcal{J}_{\mathrm{b}}^{\psi}=\mathcal{J}_{\mathrm{g}}^{\psi}, \quad \mathcal{J}_{\mathrm{b}}^{A}=\mathcal{J}_{\mathrm{g}}^{A}+\mathcal{J}_{G}^{A}+\mathcal{J}_{\Omega}^{A},
$$

and similarly for the corresponding induced currents.

We shall also be led to consider the baryonic vector and axial currents. They are defined by

$$
b_{\mu}(x) \equiv \operatorname{Tr} \gamma_{\mu} \delta S^{<}(x, x), \quad b_{\mu}^{5}(x) \equiv \operatorname{Tr} \gamma_{5} \gamma_{\mu} \delta S^{<}(x, x)
$$

and their densities will be denoted by $\mathcal{B}_{\mu}(k, X)$ and $\mathcal{B}_{\mu}^{5}(k, X)$, respectively:

$$
\mathcal{B}_{\mu}(k, X)=\operatorname{Tr} \gamma_{\mu} \delta \mathcal{S}(k, X), \quad \mathcal{B}_{\mu}^{5}(k, X)=\operatorname{Tr} \gamma_{5} \gamma_{\mu} \delta \mathcal{S}(k, X)
$$

Finally, as we shall see, a fermionic field may also induce oscillations in the axial color currents $j_{\mathrm{f}}^{5}$ and $j_{\mathrm{g}}^{5}$, with

$$
j_{\mathrm{f} \mu}^{5 a}(x) \equiv g \operatorname{Tr}\left(\gamma_{5} \gamma_{\mu} t^{a} \delta S^{<}(x, x)\right), \quad \mathcal{J}_{\mathrm{f} \mu}^{5 a}(k, X)=\operatorname{Tr}\left(\gamma_{5} \gamma_{\mu} t^{a} \delta \mathcal{S}(k, X)\right) .
$$

and similarly for $j_{\mathrm{g}}^{5}$.

We end this subsection by calculating the induced current $j_{\Omega}$ which arises from the four gluon interaction. According to eq. (2.36), $j_{\Omega}$ is proportional to the gluon loop $\left\langle A_{\mu}^{a} A_{\nu}^{b}\right\rangle_{c} \equiv D_{\mu \nu}^{a b}\left(x, x^{+}\right)$, where $D_{\mu \nu}^{a b}(x, y)$ denotes the (exact) gluon propagator. Since this is multiplied by $g^{2}$, in order to get the leading order contribution to $j_{\Omega}$ we can replace it whith the free, equilibrum propagator, given by eq. (2.48). Indeed, according to the previous discussion, the difference $D-D_{0}$ is expected to be, at most, of order $g D_{0}$. We obtain then

$$
j_{\Omega \mu}^{a}(x)=g^{2} \hat{\Gamma}_{\mu \nu \rho \lambda}^{a b c d}\left\langle A_{b}^{\nu}(x)\right\rangle D_{0 c d}^{\rho \lambda}\left(x, x^{+}\right)=-\Omega^{2}\left\langle A_{\mu}^{a}(x)\right\rangle,
$$

where we have noted

$$
\Omega^{2} \equiv(d-1) N g^{2} \int \frac{d^{3} k}{(2 \pi)^{3}} \frac{N(k)}{k}=N \frac{d-1}{12} g^{2} T^{2},
$$


and eq. (2.48) for $D_{0 \mu \nu}^{<a b}$ in Feynman gauge has been used. (Note that the ultraviolet divergence associated with the $T=0$ part of $D_{0}\left(x, x^{+}\right)$has been removed by the usual zero-temperature substraction.) The momentum space density for $j_{\Omega}$ is

$$
\mathcal{J}_{\Omega \mu}^{a}(k, X) \equiv-g N(d-1) \Delta(k)\left\langle A_{\mu}^{a}(X)\right\rangle
$$

\subsection{Equations for the 2-point functions}

In this section, the imaginary time equations of motion for the 2-point Green's functions are obtained by differentiating the mean field equations (2.18)-(2.21) with respect to the external sources. As explained in the previous subsection, we ignore all radiative corrections, that is, all terms coming from differentiating the induced sources.

We define

$$
\begin{gathered}
\mathcal{L}_{\mu \nu}^{a b}(x) \equiv\left[g_{\mu \nu} \partial_{x}^{2}-(1-\lambda) \partial_{\mu}^{x} \partial_{\nu}^{x}\right] \delta^{a b} \\
+g f^{a b c}\left[\Gamma_{\mu \nu \rho \lambda}\left\langle A_{c}^{\rho}(x)\right\rangle \partial_{x}^{\lambda}-\Gamma_{\mu \rho \lambda \nu}\left(\partial_{x}^{\lambda}\left\langle A_{c}^{\rho}(x)\right\rangle\right)\right]-g^{2} \hat{\Gamma}_{\mu \nu \rho \lambda}^{a b c d}\left\langle A_{c}^{\rho}(x)\right\rangle\left\langle A_{d}^{\lambda}(x)\right\rangle .
\end{gathered}
$$

This operator appears whenever we differentiate eq. (2.18) with respect to an arbitrary source $J$

$$
\frac{\partial}{\partial J(y)}\left\{\left[\mathcal{D}_{x}^{\nu}, \mathcal{F}_{\nu \mu}(x)\right]^{a}+\lambda \partial_{\mu} \partial_{\nu}\left\langle A_{a}^{\nu}(x)\right\rangle\right\}=\mathcal{L}_{\mu \nu}^{a b}(x) \frac{\delta\left\langle A_{b}^{\nu}(x)\right\rangle}{\delta J(y)},
$$

and it may be interpreted as the inverse propagator of a gluon in a background gauge field (see eq. (3.27) below).

In writing the equations of motion for the 2-point functions, we shall ignore, whenever they appear, the following abnormal propagators $\langle\mathrm{T} \psi(x) \psi(y)\rangle_{c},\langle\mathrm{~T} \psi(x) \zeta(y)\rangle_{c}$, $\langle\mathrm{T} \psi(x) \bar{\zeta}(y)\rangle_{c}$, together with their Hermitian conjugates. These functions are vanishing in equilibrum, while their off-equilibrum expressions involve at least one pair of weak fields; because of this, they are found to be negligible relative to the leading terms appearing next to them in the equations. In order to make this statement clear, it is useful to analyze a specific example. Consider the equation for $K_{\nu}^{a}(x, y)$ which follows from differentiating eq. (2.18) with respect to $\bar{\eta}$

$$
\begin{aligned}
\mathcal{L}_{\mu \nu}^{a b}(y) K_{b}^{\nu}(x, y) & =g S(x, y) \gamma_{\mu} t^{a}\langle\psi(y)\rangle+g\langle\bar{\psi}(y)\rangle \gamma_{\mu} t^{a}\langle\mathrm{~T} \psi(y) \psi(x)\rangle_{c} \\
& -g f^{a b c}\left(\partial_{\mu}\left\langle\bar{\zeta}^{b}(y)\right\rangle\right)\left\langle\mathrm{T} \psi(x) \zeta^{c}(y)\right\rangle_{c}-g f^{a b c}\left(\partial_{\mu}^{x}\left\langle\mathrm{~T} \psi(x) \bar{\zeta}^{b}(y)\right\rangle_{c}\right)\left\langle\zeta^{c}(y)\right\rangle .
\end{aligned}
$$

The first term in the r.h.s. of this equation involves the off-equilibrum quark propagator $S(x, y)$. We write it as $S \equiv S_{0}+\delta S$, where $S_{0}$ is the free equilibrum propagator, while 
$\delta S$ is the correction due to the average fields. We assume that $\delta S \lesssim g S_{0}$, and this will be verified explicitly in Sec.3.4. In leading order, we can therefore neglect the contribution of $\delta S$ relative to that of $S_{0}$. As mentioned, the other 2-point functions in the r.h.s. of eq. (3.20) are also expected to be small with respect to $S_{0}$. We show how to verify this explicitly for the first of them, which is proportional to $\langle\mathrm{T} \psi(x) \psi(y)\rangle_{c}$. By differentiating eq. (2.19) with respect to $\bar{\eta}$, we obtain the equation

$$
\mathcal{D}_{x}\langle\mathrm{~T} \psi(x) \psi(y)\rangle_{c}=i g K_{\nu}^{a}(y, x) \gamma^{\nu} t^{a}\langle\psi(x)\rangle
$$

As we shall see in Sec.3.4 (after eq. (3.47)), this equation implies that $\langle\mathrm{T} \psi(x) \psi(y)\rangle_{c} \sim$ $\left(S_{0} / T^{3}\right)\langle\psi\rangle^{2}$. Therefore, the second term in the r.h.s. of eq. (3.20) is $g\langle\bar{\psi}(y)\rangle \gamma_{\mu} t^{a}$ $\langle\mathrm{T} \psi(y) \psi(x)\rangle_{c} \sim g S_{0}\left(\langle\bar{\psi}\rangle\langle\psi\rangle / T^{3}\right)\langle\psi\rangle$. Relative to the first term $g S_{0}(x-y) \gamma_{\mu} t^{a}\langle\psi(y)\rangle$, this is of order $(\langle\bar{\psi}\rangle\langle\psi\rangle) / T^{3}$, that is, at most of order $g$. Similar considerations apply to the last two terms in eq. (3.20), which can be also neglected. Our final equation for $K_{\nu}^{a}(x, y)$ is therefore

$$
\mathcal{L}_{\mu \nu}^{a b}(y) K_{b}^{\nu}(x, y)=g S_{0}(x-y) \gamma_{\mu} t^{a}\langle\psi(y)\rangle
$$

The previous arguments may be extended to all the other equations of interest. We shall not repeat them, but simply list the equations obtained after the corresponding simplifications have been done.

By differentiating again eq. (2.19), but this time with respect to $j_{\nu}^{b}$, we derive a second equation for $K_{\nu}^{b}(x, y)$

$$
\mathcal{D}_{x} K_{b}^{\nu}(x, y)=-i g t^{a} \gamma_{\mu}\langle\psi(x)\rangle D_{0 a b}^{\mu \nu}(x-y)
$$

A diagrammatic interpretation of eqs. (3.22) and (3.23) is given in Fig.4. There are, of course, similar equations for $H_{\nu}^{a}$, but we do not write them explicitly, because they are simply related (by Hermitian conjugation) to eqs. (3.22) and (3.23).

The equation for $S(x, y)$ is obtained by differentiating eq. (2.19) with respect to $\eta$

$$
\mathcal{P}_{x} S(x, y)=i \delta\left(\tau_{x}-\tau_{y}\right) \delta^{3}(\mathbf{x}-\mathbf{y})-i g \gamma^{\nu} t^{a}\langle\psi(x)\rangle H_{\nu}^{a}(x, y)
$$

The diagrammatic contributions to $S$ are displayed in Fig.5.

By differentiating the equations $(2.20)$ and (2.21) for the ghost fields with respect $C^{b}$ and $\bar{C}^{a}$, we generate two equations for $G^{a b}(x, y)$ :

$$
\begin{aligned}
& \partial_{x}^{2} G^{a b}(x, y)-g f^{a c d}\left\langle A_{\mu}^{c}(x)\right\rangle \partial_{x}^{\mu} G^{d b}(x, y)= \\
& \quad \delta^{a b} \delta\left(\tau_{x}-\tau_{y}\right) \delta^{3}(\mathbf{x}-\mathbf{y})-g f^{a c d} \partial_{x}^{\mu}\left[\left\langle\zeta^{c}(x)\right\rangle \bar{O}_{\mu}^{d b}(x, y)\right],
\end{aligned}
$$


and

$$
\begin{aligned}
& \partial_{y}^{2} G^{a b}(x, y)-g f^{b c d}\left\langle A_{\mu}^{c}(y)\right\rangle \partial_{y}^{\mu} G^{a d}(x, y)= \\
& \delta^{a b} \delta\left(\tau_{x}-\tau_{y}\right) \delta^{3}(\mathbf{x}-\mathbf{y})-g f^{b c d}\left(\partial^{\mu}\left\langle\bar{\zeta}^{c}(y)\right\rangle\right) O_{\mu}^{a d}(x, y) .
\end{aligned}
$$

Finally, by differentiating eq. (2.18) with respect to $j_{b}^{\nu}$, we get the following equation for $D_{\mu \nu}^{a b}(x, y)$ :

$$
\begin{aligned}
\mathcal{L}_{\mu \sigma}^{a c}(x) D_{c \nu}^{\sigma b}(x, y) & =g_{\mu \nu} \delta^{a b} \delta\left(\tau_{x}-\tau_{y}\right) \delta^{3}(\mathbf{x}-\mathbf{y}) \\
& +g\left\{\langle\bar{\psi}(x)\rangle \gamma_{\mu} t^{a} K_{\nu}^{b}(x, y)+H_{\nu}^{b}(y, x) \gamma_{\mu} t^{a}\langle\psi(x)\rangle\right\} \\
& -g f^{a c d}\left\{\left(\partial_{\mu}\left\langle\bar{\zeta}^{c}(x)\right\rangle\right) O_{\nu}^{d b}(x, y)+\left(\partial_{\mu}^{x} \bar{O}_{\nu}^{b c}(y, x)\right)\left\langle\zeta^{d}(x)\right\rangle\right\},
\end{aligned}
$$

Diagrammatic contributions to $D$ are shown in Fig.6.

We have not written here the corresponding equations for the ghost-gluon abnormal propagators, $O_{\nu}^{a b}$ and $\bar{O}_{\nu}^{a b}$. We do this in Appendix A, where we prove that, in leading order, the equation of motion for the ghost mean fields coincide with the tree level equations; there is no collective excitation with ghost quantum numbers. Accordingly, we shall not be concerned anymore in the main text with the propagation of the ghost fields. We set the ghost sources $C^{a}$ and $\bar{C}^{a}$ to zero. Then $\langle\zeta\rangle$ and $\langle\bar{\zeta}\rangle$ vanish, and all terms involving $O_{\nu}^{a b}$ and $\bar{O}_{\nu}^{a b}$ disappear from eqs. (3.25)-(3.27).

In what follows, we simplify the writing and denote the average gauge potential or the average fermionic field respectively by $A(x)$ and $\psi(x)$, omitting the brackets. We also denote simply by $D$ or $F_{\mu \nu}$ the covariant derivative or the field strength tensor associated to the average gauge field, in place of $\mathcal{D}$ or $\mathcal{F}_{\mu \nu}$. Calligraphic capitals will therefore be reserved for Wigner functions and for the densities of the induced sources. Furthermore, we shall restrict our discussion in the main text to the Feynman gauge $(\lambda=1)$; we prove in Appendix B that our final equations are independent of the gauge fixing parameter.

The calculation of the induced sources from the 2-point functions given by the previous equations is equivalent to the calculation of the one-loop Feynman diagrams displayed in Figs.7 and 8. The approximations to be implemented in the rest of this section will provide us with consistent equations from which the leading contributions to these diagrams will be directly obtained.

\subsection{Equations for $\mathcal{K}$ and $\mathcal{H}$}

The real-time equations which determine the abnormal quark-gluon propagator $K_{b}^{\nu}$ are obtained from the imaginary-time equations (3.22) and (3.23). We consider $\tau_{y}>\tau_{x}$ and 
obtain, after analytic continuation,

$$
\mathcal{L}_{\mu \nu}^{a b}(y) K_{b}^{\nu<}(x, y)=-g S_{0}^{<}(x-y) \gamma_{\mu} t^{a} \psi(y)
$$

and

$$
\not D_{x} K_{b}^{\nu<}(x, y)=-i g t^{a} \gamma_{\mu} \psi(x) D_{0 a b}^{<\mu \nu}(x-y)
$$

By acting on eq. (3.29) with the differential operator $\not D_{x}$ from the left, we obtain

$$
\not D_{x} \not D_{x} K_{a}^{\nu<}(x, y)=i g \not D_{x}\left[t^{a} \gamma^{\nu} \psi(x) \Delta(x-y)\right]
$$

To simplify the 1.h.s. of this equation, we use the identity

$$
\not D_{x} \not D_{x}=D_{x}^{2}+\frac{g}{2} \sigma^{\mu \nu} F_{\mu \nu}(x)
$$

where $\sigma^{\mu \nu}=(i / 2)\left[\gamma^{\mu}, \gamma^{\nu}\right]$ and

$$
D_{x}^{2}=\partial_{x}^{2}+2 i g A(x) \cdot \partial_{x}+i g(\partial \cdot A(x))-g^{2} A^{2}(x) .
$$

Then we replace $x$ and $y$ by the coordinates $s$ and $X$ (see eq. (3.4)) and rewrite the derivatives as $\partial_{x}=\partial_{s}+\frac{1}{2} \partial_{X}, \partial_{y}=-\partial_{s}+\frac{1}{2} \partial_{X}$ and $\partial_{x}^{2}-\partial_{y}^{2}=2 \partial_{s} \cdot \partial_{X}$. We recall that a derivative $\partial_{X}$ is to be regarded as being of order $g T$, and a derivative $\partial_{s}$ as being of order $T$. Furthermore, the mean field is assumed to be of order $T$, that is $g A$ is to be considered of the same order as the derivative $\partial_{X}$, which insures the consistency of the soft covariant derivative, $D_{X}=\partial_{X}+i g A$. As for $g F_{\mu \nu}$, it is of order $g^{2} T^{2}$. Finally, we expand $A(x) \equiv A(X+s / 2) \approx A(X)+(s / 2) \cdot \partial_{X} A(X)$, where the second term is $g$ times the first one, and likewise for the other fields. Finally, eq. (3.30) reduces to

$$
\left[\partial_{s}^{2}+\partial_{s} \cdot \partial_{X}+2 i g A(X) \cdot \partial_{s}\right] K_{a}^{\mu<}(s, X)=i g\left(\not \partial_{s} \Delta\right) \gamma^{\mu} t^{a} \psi(X) .
$$

Similar manipulations performed on eq. (3.28) lead to

$$
\begin{gathered}
{\left[\delta^{a b} g_{\mu \nu}\left(\partial_{s}^{2}-\partial_{s} \cdot \partial_{X}\right)-g f^{a b c} \Gamma_{\mu \nu \rho \lambda} A_{c}^{\rho}(X) \partial_{s}^{\lambda}\right] K_{b}^{\nu<}(s, X)} \\
=-g S_{0}^{<}(s) \gamma_{\mu} t^{a} \psi(X) .
\end{gathered}
$$

We remark that the contribution from the four gluon interaction (i.e., the term proportional to $\hat{\Gamma}$ ) disappears at this order.

In writing eqs. (3.33) and (3.34), we have neglected terms of order $g^{2} T^{2}$ inside the brackets on the left hand sides. As for the remaining terms, the first one, $\partial_{s}^{2}$, is of order $T^{2}$, while all the others are of order $g T^{2}$. Therefore, in order to have consistent equations 
order by order in $g$, we should also expand $K^{<}=K^{(0)}+K^{(1)}+\ldots$, with $K^{(1)} \sim g K^{(0)}$, etc... Of course, because of the approximations which have been done, eqs. (3.33) and (3.34) can only be used to determine $K^{(0)}$. The dominant terms in the two equations lead to the consistency condition

$$
\partial_{s}^{2} K^{(0)}(s, X)=0 .
$$

Next, we note that the "big" derivatives $\partial_{s}^{2} \sim T^{2}$ give rise to contributions of the form $\partial_{s}^{2} K^{(1)}$ which are of the same order as, for example, $\left(\partial_{s} \cdot \partial_{X}\right) K^{(0)}$. Such terms are inconsistent and, besides, they cannot be easily evaluated. In order to do so, one would need equations for $K^{(1)}$ containing the unknown function $K^{(2)}$, and so forth. However, such "spurious" contributions cancel in the difference of the two equations (3.33) and (3.34). By doing this difference, we end up with the following equation for $K^{(0)}$, which we denote from now on simply by $K^{<}$:

$$
\begin{gathered}
{\left[\delta^{a b} g_{\mu \nu}\left(\partial_{X}+i g A(X)\right) \cdot \partial_{s}+\frac{g}{2} f^{a b c} \Gamma_{\mu \nu \rho \lambda} A_{c}^{\rho}(X) \partial_{s}^{\lambda}\right] K_{b}^{\nu<}(s, X)} \\
=\frac{g}{2}\left[S_{0}^{<}(s)+i \not_{s} \Delta(s)\right] \gamma_{\mu} t^{a} \psi(X) .
\end{gathered}
$$

When Fourier transformed with respect to $s$, eq. (3.36) translates into an equation for the Wigner function $\mathcal{K}_{\mu}^{a}(k, X)$ :

$$
\begin{gathered}
k \cdot\left(\partial_{X}+i g A(X)\right) \mathcal{K}_{\mu}^{a}(k, X)+\frac{g}{2} f^{a b c} \Gamma_{\mu \nu \rho \lambda} A_{c}^{\rho}(X) k^{\lambda} \mathcal{K}_{b}^{\nu}(k, X) \\
=i \frac{g}{2} \rho_{0}(k)\left[N\left(k_{0}\right)+n\left(k_{0}\right)\right] k \gamma_{\mu} t^{a} \psi(X) .
\end{gathered}
$$

From the right hand side of eq. (3.37), one sees that $\mathcal{K}_{\mu}^{a}$ is proportional to $\not k \delta\left(k^{2}\right)$. Consequently

$$
k^{2} \mathcal{K}_{\mu}^{a}(k, X)=0, \quad \not k \mathcal{K}_{\mu}^{a}(k, X)=0 .
$$

The first identity above is the consistency condition (3.35). It simply expresses the massshell condition for $\mathcal{K}_{\mu}^{a}$. The second identity is the Dirac equation for the quark-gluon propagator. It can be also verified that the right hand side of eq. (3.37) is transverse with respect to $k$, so that

$$
k^{\mu} \mathcal{K}_{\mu}^{a}(k, X)=0 .
$$

By using these properties, namely, Eqs.(3.38) and (3.39), one easily transforms eq. (3.37) into an equation for $\mathbb{K}^{a}(k, X) \equiv \gamma^{\mu} \mathcal{K}_{\mu}^{a}(k, X)$

$$
\begin{gathered}
k \cdot\left[\left(\partial_{X}+i g A(X)\right) \delta^{a b}+i g \tilde{A}^{a b}(X)\right] \mathbb{K}^{b}(k, X) \\
=-i g \frac{d-2}{2} \rho_{0}(k)\left[N\left(k_{0}\right)+n\left(k_{0}\right)\right] \not k t^{a} \psi(X),
\end{gathered}
$$


where the factor $(d-2)$ results from the identity $\gamma^{\mu} k \gamma_{\mu}=-(d-2) \not k$. Eq. (3.40) is covariant with respect to local gauge transformations of the mean fields $\psi$ and $A$. This is seen by noting that the differential operator from the left hand side is a covariant derivative acting on vectors in the direct product of the fundamental and the adjoint representations. It follows that $\mathbb{K}^{a}$ transforms as $t^{a} \psi(x)$ under a gauge transformation. Explicitly, when $\psi(x) \rightarrow S(x) \psi(x)$, with $S(x)=\exp \left(i \theta^{a}(x) t^{a}\right)$, then

$$
\mathbb{K}_{i}^{a}(k, X) \rightarrow \tilde{S}_{a b}(X) S_{i j}(X) K_{j}^{b}(k, X),
$$

where $\tilde{S}(x)=\exp \left(i \theta^{a}(x) T^{a}\right)$ is the gauge transformation in the adjoint representation.

The equation satisfied by $\not{K}(k, X) \equiv t^{a} \not_{k}^{a}(k, X)$ results from eq. (3.40) after multiplication by $t^{a}$

$$
\left(k \cdot D_{X}\right) \not K(k, X)=-i g \frac{d-2}{2} C_{f} \rho_{0}(k)\left[N\left(k_{0}\right)+n\left(k_{0}\right)\right] \not k \psi(X),
$$

where $C_{f}$ is the quark Casimir. The covariant derivative which remains in the left hand side of eq. (3.42) is that of the fundamental representation, $D_{X}$, showing that $\not K$ transforms as $\psi$. In contrast with what happens in eq. (3.40) for $\mathbb{K}^{a}$, the gauge field insertions on the hard gluon lines have disappeared in eq. (3.42), which coincides with the corresponding equation for an abelian plasma [16].

We end this section by writing the equation satisfied by the Wigner transform of the quark-gluon propagator $H_{\mu}^{a<}$. This follows from eq. (3.42), by noting that $\mathcal{H}(k, X)=$ $\mathbb{K}^{\dagger}(k, X) \gamma^{0}$,

$$
\mathcal{H}(k, X)\left(k \cdot D_{X}^{\dagger}\right)=i g \frac{d-2}{2} C_{f} \rho_{0}(k)\left[N\left(k_{0}\right)+n\left(k_{0}\right)\right] \bar{\psi}(X) \not k .
$$

Here $D_{\mu}^{\dagger} \equiv \partial_{\mu}^{\dagger}-i g A_{\mu}$, where the derivative operator $\partial_{\mu}^{\dagger} \equiv\left(\overleftarrow{\partial} / \partial x^{\mu}\right)$ acts on its left.

\subsection{Equations for $\delta \mathcal{S}_{A}$ and $\delta \mathcal{S}_{\psi}$}

The equations for the off-equilibrium fermion propagator are obtained by analytic continuation from eq. (3.24) and its conjugate:

$$
\begin{gathered}
\not D_{x} S^{<}(x, y)=i g \gamma^{\nu} t^{a} \psi(x) H_{\nu}^{a<}(x, y), \\
S^{<}(x, y) \not D_{y}^{\dagger}=-i g K_{\nu}^{a<}(x, y) \bar{\psi}(y) t^{a} \gamma^{\nu},
\end{gathered}
$$

with $\not D^{\dagger} \equiv \not^{\dagger}-i g A$ and $\not^{\dagger} \equiv\left(\overleftarrow{\partial} / \partial x^{\mu}\right) \gamma^{\mu}$. We transform them by first acting with the covariant derivatives $D_{x}$ on eq. (3.44) from the left, and with $\not_{y}^{\dagger}$ on eq. (3.45) from 
the right. We subtract the resulting equations and separate the leading order terms by manipulations similar to those encountered in the previous subsection. A new feature stems from the fact that in equilibrium $S^{<}(x, y)$ does not vanish, but is equal to $S_{0}^{<}$, eq. (2.46). As explained in Sec.3.1, we write $S^{<}(x, y)=S_{0}^{<}(x-y)+\delta S(x, y)$ and assume $\delta S$ to be of order $g S_{0}^{<}$. It is then straightforward to derive the equation satisfied in leading order by the Wigner function $\delta \mathcal{S}(k, X)$,

$$
\begin{array}{r}
{\left[k \cdot D_{X}, \delta \mathcal{S}(k, X)\right]=-i \frac{g}{4} F^{\mu \nu}\left[\sigma_{\mu \nu}, S_{0}^{<}(k)\right]-g\left(\partial_{X}^{\nu} A^{\mu}\right) k_{\mu} \partial_{\nu}^{k} S_{0}^{<}(k)} \\
-i \frac{g}{2}\left\{\mathcal{K}_{\nu}^{a}(k, X) \bar{\psi}(X) t^{a} \gamma^{\nu} \not k-\not k \gamma^{\nu} t^{a} \psi(X) \mathcal{H}_{\nu}^{a}(k, X)\right\} .
\end{array}
$$

We call $\delta \mathcal{S}^{A}(k, X)$ the solution of this equation when $\psi=\bar{\psi}=0$, and $\delta \mathcal{S}^{\psi}(k, X)$ the correction which includes the effects of the fermionic fields. We have $\delta \mathcal{S}(k, X)=$ $\delta \mathcal{S}^{A}(k, X)+\delta \mathcal{S}^{\psi}(k, X)$, and

$$
\left[k \cdot D_{X}, \delta \mathcal{S}^{\psi}(k, X)\right]=-i \frac{g}{2}\left\{\mathcal{K}_{\nu}^{a}(k, X) \bar{\psi}(X) t^{a} \gamma^{\nu} \not k-\not k \gamma^{\nu} t^{a} \psi(X) \mathcal{H}_{\nu}^{a}(k, X)\right\} .
$$

By using this equation, together with eqs. (3.42) and (3.43), we can verify that $\delta \mathcal{S}^{\psi}(k, X) \sim$ $\left(S_{0} / T^{3}\right) \bar{\psi} \psi$. Thus $\delta \mathcal{S}^{\psi}(k, X) \lesssim g S_{0}$ when $\bar{\psi} \psi \lesssim g T^{3}$, as anticipated in Sec.3.1. Remark also the similarity between eqs. (3.44)-(3.45) for $S^{<}(x, y)$ and eq. (3.21) for the abnormal propagator $\langle\mathrm{T} \psi(x) \psi(y)\rangle_{c}$. By following the same steps as above, one can derive for the Wigner transform of $\langle\mathrm{T} \psi(x) \psi(y)\rangle_{c}$ an equation analogous to eq. (3.47) for $\delta \mathcal{S}^{\psi}(k, X)$. From it we derive the estimate $\langle\psi \psi\rangle_{c} \sim\left(S_{0} / T^{3}\right) \psi^{2}$ which has been used in Sec.3.3 in order to discard the contribution of such abnormal propagators.

As a color matrix acting in the fundamental representation, $\delta \mathcal{S}^{\psi}$ can be decomposed into a color singlet and a color octet: $\delta \mathcal{S}^{\psi} \equiv \delta \mathcal{S}_{\mathrm{o}}^{\psi}+\delta \mathcal{S}_{a}^{\psi} t^{a}$. Besides, $\delta \mathcal{S}^{\psi}$ is a $4 \times 4$ matrix in Dirac indices. We can verify on eq. (3.47) that $\operatorname{tr} \delta \mathcal{S}^{\psi}=\operatorname{tr} \gamma_{5} \delta \mathcal{S}^{\psi}=\operatorname{tr} \sigma_{\mu \nu} \delta \mathcal{S}^{\psi}=0$, and these equalities hold for both the color singlet and the color octet (the trace acts on Dirac indices only). The quantities $\operatorname{tr} \gamma_{\mu} \delta \mathcal{S}^{\psi}$ and $\operatorname{tr} \gamma_{5} \gamma_{\mu} \delta \mathcal{S}^{\psi}$ are nonvanishing. Thus a fermionic field induces oscillations in the vector and axial baryonic currents $b_{\mu}$ and $b_{\mu}^{5}$, eq. (3.12), as well as in the vector and axial color currents, $j_{\mathrm{f} \mu}^{a}$ and $j_{\mathrm{f} \mu}^{5 a}$. The corresponding densities are given in eqs. (3.13) and (3.14), with $\delta \mathcal{S}$ replaced by $\delta \mathcal{S}^{\psi}(k, X)$.

Let us consider now the equation satisfied by $\delta \mathcal{S}^{A}(k, X)$. By using eq. (2.46), we can write it as follows:

$$
\left[k \cdot D_{X}, \delta \mathcal{S}^{A}(k, X)\right]=-g\left\{\left[k \cdot D_{X}, A\right]+\not k k_{\mu}\left(\partial_{X}^{\nu} A^{\mu}\right) \partial_{\nu}\right\} \tilde{\Delta}(k) .
$$

This equation shows that $\delta \mathcal{S}^{A} \sim g(A / T) S_{0}$, so that $\delta \mathcal{S}^{A} \sim g S_{0}$ when $A \sim T$. It can also be verified that $\delta \mathcal{S}^{A} \propto \gamma_{\mu} t^{a}\left(\operatorname{Tr} \gamma^{\mu} t^{a} \delta \mathcal{S}^{A}\right)$. Thus, a gauge field induces only fluctuations in the vector color current. 
Eq. (3.48) is not covariant under local gauge transformations. This reflects the lack of covariance of our definition for the fermionic Wigner function $\delta \mathcal{S}(k, X)$. We could, instead, define a gauge covariant Wigner transform, as for example in ref. [22]. But it is easier to recover gauge covariance directly at the level of eq. (3.48). This can be done in a standard way, by replacing the canonical momentum $k$, associated with the gradient operator, by the kinetic momentum $p \equiv k-g A$, related to the particle velocity. By applying this operation to the quark Wigner transform $\mathcal{S}(k, X)$, we obtain a new function of $p$ and $X, \mathcal{S}(p, X) \equiv \mathcal{S}(k=p+g A, X)$, which transforms covariantly. This can be verified directly by writing (for $\psi=\bar{\psi}=0) \mathcal{S}(p, X) \equiv S_{0}^{<}(p)+\delta \dot{\mathcal{S}}^{A}(p, X)$. Then, in leading order,

$$
\delta \dot{\mathcal{S}}^{A}(p, X)=\delta \mathcal{S}^{A}(p, X)+g\left(A(X) \cdot \partial_{p}\right) S_{0}^{<}(p),
$$

and $\delta \mathcal{S}^{A}(p, X)$ satisfies the gauge covariant equation

$$
\left[p \cdot D_{X}, \delta \dot{\mathcal{S}}^{A}(p, X)\right]=g \not p p \cdot F(X) \cdot \partial_{p} \tilde{\Delta}(p),
$$

deduced from eq. (3.48). This is the non-abelian generalization of the well-known linearized Vlasov equation. Note that although $g A \sim g T \ll k \sim T$, the change from $k$ to $p$ in $\mathcal{S}(k, X)$ is non trivial, as the second term in eq. (3.49) is of the same order of magnitude as the first one. However, this term is a total derivative with respect to $p$, so that it does not contribute to the induced current $j_{\mathrm{f}}$ (see eq. (3.5)).

The change from canonical to kinetic momentum in $\delta \mathcal{S}^{\psi}(k, X)$ is irrelevant at this order. Indeed, if we replace $k$ by $p=k-g A$ in eq. (3.47), we only generate terms of higher order. And, in fact, eq. (3.47) is gauge covariant as it stands.

In summary, the previous discussion shows that all effects induced by the mean fields on the hard quarks can be described by the gauge covariant Wigner function

$$
\mathcal{\mathcal { S }}(k, X) \equiv S_{0}^{<}(k)+\delta \mathcal{S}_{\mathrm{o}}^{\psi}(k, X)+\left(\delta \mathcal{S}_{a}^{\psi}(k, X)+\delta \dot{\mathcal{S}}_{a}^{A}(k, X)\right) t^{a},
$$

and $k$ is to be understood here and from now on as the kinetic momentum. In particular, the induced current $j_{\mathrm{f}}$ has the gauge covariant density

$$
\mathcal{J}_{\mathrm{f} \mu}^{a}(k, X)=\operatorname{Tr} \gamma_{\mu} t^{a}\left(N_{\mathrm{f}} \delta \mathcal{S}^{A}(k, X)+\delta \mathcal{S}^{\psi}(k, X)\right) \equiv \mathcal{J}_{\mathrm{f} \mu}^{A a}(k, X)+\mathcal{J}_{\mathrm{f} \mu}^{\psi a}(k, X) .
$$

The factor $N_{\mathrm{f}}$ reflects the fact that all quark flavors contribute identically to the current induced by the average gauge fields. Similarly, eq. (3.47) contained an implicit sum over the different flavors of the quark mean fields. 


\subsection{Equations for $\delta \mathcal{G}$}

From eqs. (3.25) and (3.26), one obtains the following real-time equations of motion for the ghost propagator

$$
\partial_{x}^{2} G_{a b}^{<}(x, y)=g f^{a c d} A_{\mu}^{c}(x) \partial_{x}^{\mu} G_{d b}^{<}(x, y)
$$

and

$$
\partial_{y}^{2} G_{a b}^{<}(x, y)=g f^{b c d} A_{\mu}^{c}(y) \partial_{y}^{\mu} G_{a d}^{<}(x, y) .
$$

After separating the induced part, by setting $G_{a b}^{<}(s, X)=G_{0 a b}^{<}(s)+\delta G_{a b}(s, X)$, with $G_{0 a b}^{<}$given by eq. (2.49), we transform these equations in a way which should by now be familiar. We obtain

$$
\begin{gathered}
k \cdot\left[\partial_{X} \delta \mathcal{G}_{a b}(k, X)+\frac{g}{2}\left(f^{a c d} \delta \mathcal{G}_{c b}+f^{b c d} \delta \mathcal{G}_{a c}\right) A^{d}(X)\right] \\
=i \frac{g}{2} f^{a b c}\left(\partial_{\nu} A_{\mu}^{c}(X)\right) k^{\mu}\left(\partial_{k}^{\nu} \Delta\right)
\end{gathered}
$$

where $\delta \mathcal{G}(k, X)$ is the Wigner transform of $\delta G(s, X)$. This equation shows that, as expected, $\delta \mathcal{G} \sim g(A / T) \Delta$.

For later reference, we note that $\delta \mathcal{G}_{a b}$ determined by eq. (3.55) is a color matrix of the adjoint representation, i.e., it can be written as $\delta \mathcal{G}_{a b} \equiv\left(T^{c}\right)_{a b} \delta \mathcal{G}_{c}$, where the new functions $\delta \mathcal{G}_{a}(k, X)$ satisfy

$$
\left(k \cdot \partial_{X}\right) \delta \mathcal{G}_{a}(k, X)-\frac{g}{2} f^{a b c}\left(k \cdot A_{b}(X)\right) \delta \mathcal{G}_{c}(k, X)=-\frac{g}{2}\left(\partial_{\nu} A_{\mu}^{a}(X)\right) k^{\mu}\left(\partial_{k}^{\nu} \Delta\right) .
$$

Then the ghost contribution to the induced current density may be written as (see eq. (2.42))

$$
\mathcal{J}_{G \mu}^{a}(k, X)=-i k_{\mu} f^{a b c} \delta \mathcal{G}_{b c}(k, X)=-N k_{\mu} \delta \mathcal{G}^{a}(k, X)
$$

\subsection{Equations for $\delta \mathcal{D}_{A}$ and $\delta \mathcal{D}_{\psi}$}

After analytic continuation to real time, eq. (3.27) gives

$$
\mathcal{L}_{\mu \sigma}^{a c}(x) D_{c \nu}^{\sigma b<}(x, y)=g\left\{\bar{\psi}(x) \gamma_{\mu} t^{a} K_{\nu}^{b<}(x, y)+H_{\nu}^{b>}(y, x) \gamma_{\mu} t^{a} \psi(x)\right\} .
$$

Another equation for $D^{<}$is obtained by interchanging in this equation the space-time arguments, as well as the color and Minkowski indices,

$$
\mathcal{L}_{\nu \sigma}^{b c}(y) D_{\mu c}^{a \sigma<}(x, y)=g\left\{\bar{\psi}(y) \gamma_{\nu} t^{b} K_{\mu}^{a>}(y, x)+H_{\mu}^{a<}(x, y) \gamma_{\nu} t^{b} \psi(y)\right\}
$$


The symmetry property $D_{\mu \nu}^{a b<}(x, y)=D_{\nu \mu}^{b a>}(y, x)$ has been used. We set $D_{\mu \nu}^{a b<}(x, y) \equiv$ $-g_{\mu \nu} \delta^{a b} \Delta(s)+\delta D_{\mu \nu}^{a b}(s, X)$, with $\delta D$ expected to be of order $g \Delta$, and we separate the leading order terms in eqs. (3.58)-(3.59) in order to get equations for the Wigner function $\delta \mathcal{D}_{\mu \nu}^{a b}(k, X)$. In order to do this, we need all the analytic pieces of the abnormal quarkgluon propagators, i.e., not only $K^{<}$and $H^{<}$, for which we have obtained equations in Sec.3.3, but also $K^{>}$and $H^{>}$. In fact, by repeating the calculations of Sec.3.3, one can show that, in the present approximation, $K^{>}$and $K^{<}$satisfy identical equations, and both vanish in equilibrium, so that

$$
\mathcal{K}_{\mu}^{a>}(k, X) \approx \mathcal{K}_{\mu}^{a<}(k, X) \equiv \mathcal{K}_{\mu}^{a}(k, X),
$$

and similarly for the $\mathcal{H}$ functions.

In order to separate the effects of gauge fields from those of fermionic fields, we write $\delta \mathcal{D} \equiv \delta \mathcal{D}^{A}+\delta \mathcal{D}^{\psi}$, where $\delta \mathcal{D}^{A}(k, X)$ is the modification induced by the gauge field when $\psi=\bar{\psi}=0$. At leading order in $g$, eqs. (3.58) and (3.59) imply the consistency conditions

$$
k^{2} \delta \mathcal{D}_{\mu \nu}^{A a b}(k, X)=i g f^{a b c} \Delta(k) \Gamma_{\mu \nu \rho \lambda} A_{a}^{\rho}(X) k^{\lambda}, \quad k^{2} \delta \mathcal{D}_{\mu \nu}^{\psi a b}=0 .
$$

These will be used later in simplifying some formulae. At next to leading order, we take the difference of eqs. (3.58) and (3.59) and obtain, after a lengthy, but straightforward calculation,

$$
\begin{gathered}
k \cdot \partial_{X} \delta \mathcal{D}_{\mu \nu}^{A a b}-\frac{g}{2}\left(f^{a c d} \Gamma_{\mu \sigma \lambda \rho} \delta \mathcal{D}_{d \nu}^{A \sigma b}+f^{b c d} \Gamma_{\nu \sigma \lambda \rho} \delta \mathcal{D}_{\mu d}^{A a \sigma}\right) k^{\lambda} A_{c}^{\rho} \\
=-i \frac{g}{2} f^{a b c} \Gamma_{\mu \nu \rho \lambda}\left(\partial_{X}^{\sigma} A_{c}^{\rho}\right) k^{\lambda}\left(\partial_{\sigma}^{k} \Delta\right)
\end{gathered}
$$

and

$$
\begin{aligned}
& k \cdot \partial_{X} \delta \mathcal{D}_{\mu \nu}^{\psi a b}-\frac{g}{2}\left(f^{a c d} \Gamma_{\mu \sigma \lambda \rho} \delta \mathcal{D}_{d \nu}^{\psi \sigma b}+f^{b c d} \Gamma_{\nu \sigma \lambda \rho} \delta \mathcal{D}_{\mu d}^{\psi a \sigma}\right) k^{\lambda} A_{c}^{\rho} \\
& =i \frac{g}{2} \bar{\psi}\left\{\gamma_{\mu} t^{a} \mathcal{K}_{\nu}^{b}-\gamma_{\nu} t^{b} \mathcal{K}_{\mu}^{a}\right\}+i \frac{g}{2}\left\{\mathcal{H}_{\nu}^{b} \gamma_{\mu} t^{a}-\mathcal{H}_{\mu}^{a} \gamma_{\nu} t^{b}\right\} \psi
\end{aligned}
$$

Note that the four gluons vertex does not contribute to this order and in this particular gauge, so that there are no terms quadratic in $A$ in the right hand side of eq. (3.62).

The contribution of hard gluons to the induced current can be calculated from eq. (2.43), which leads to the density

$$
\mathcal{J}_{\mathrm{g} \mu}^{a}(k, X)=i f^{a b c} \Gamma_{\mu \rho \lambda \nu} k^{\lambda} \delta \mathcal{D}_{b c}^{\rho \nu}(k, X) .
$$

The separation $\delta \mathcal{D}=\delta \mathcal{D}^{A}+\delta \mathcal{D}^{\psi}$ leads to a corresponding separation in the induced current density, $\mathcal{J}_{\mathrm{g}}=\mathcal{J}_{\mathrm{g}}^{A}+\mathcal{J}_{\mathrm{g}}^{\psi}$. We are interested now in transforming the previous equations for $\delta \mathcal{D}^{A}$ and $\delta \mathcal{D}^{\psi}$ into equations for these densities. 
Let us consider first the density $\mathcal{J}_{\mathrm{g}}^{\psi}$. This is obtained from $\delta \mathcal{D}^{\psi}$, given by eq. (3.63) above. Since the r.h.s. of this equation is antisymmetric with respect to simultaneous permutations of the color and Minkovski indices, the same property holds for $\delta \mathcal{D}^{\psi}$

$$
\delta \mathcal{D}_{\mu \nu}^{\psi a b}(k, X)=-\delta \mathcal{D}_{\nu \mu}^{\psi b a}(k, X)
$$

Furthermore, the right hand side of eq. (3.63) is transverse with respect to $k$. Indeed, by taking its scalar product with $k^{\mu}$, we find in the r.h.s. combinations like $k \cdot \mathcal{K}^{a}$ or $\not k \mathcal{K}_{\mu}^{a}$ which are vanishing in the present approximation, as shown by eqs. (3.38) and (3.39). This implies

$$
k^{\mu} \delta \mathcal{D}_{\mu \nu}^{\psi a b}(k, X)=\delta \mathcal{D}_{\mu \nu}^{\psi a b}(k, X) k^{\nu}=0
$$

Using this property, together with the definition (3.64) for the current density $\mathcal{J}_{\mathrm{g}}^{\psi}$, as well as the explicit form of the $\Gamma$ symbol, eq. (2.13), one obtains

$$
\mathcal{J}_{\mathrm{g} \mu}^{\psi a}(k, X)=-i k_{\mu} f^{a b c}\left(\delta \mathcal{D}_{b c}^{\psi}\right)_{\nu}^{\nu}(k, X)
$$

By using the transversality property (3.66), we readily derive from eq. (3.63) the following equation for $\left(\delta \mathcal{D}_{a b}^{\psi}\right)_{\nu}^{\nu}$ :

$$
\begin{array}{r}
k \cdot \partial_{X}\left(\delta \mathcal{D}_{a b}^{\psi}\right)_{\nu}^{\nu}-g\left(k \cdot A_{c}\right)\left\{f^{a c d}\left(\delta \mathcal{D}_{d b}^{\psi}\right)_{\nu}^{\nu}+f^{b c d}\left(\delta \mathcal{D}_{a d}^{\psi}\right)_{\nu}^{\nu}\right\} \\
=i \frac{g}{2} \bar{\psi}\left\{t^{a} \mathbb{K}^{b}-t^{b} \mathbb{K}^{a}\right\}+i \frac{g}{2}\left\{\mathcal{H}^{b} t^{a}-\mathcal{H}^{a} t^{b}\right\} \psi .
\end{array}
$$

From eq. (3.40), one can show that

$$
t^{a} \mathbb{K}^{b}-t^{b} \mathbb{K}^{a}=i f^{a b c} \mathbb{K}^{c}=-T^{c} \mathbb{K}^{c} .
$$

It then follows from eq. (3.68) that $\left(\delta \mathcal{D}_{a b}^{\psi}\right)_{\nu}^{\nu}$ is a color matrix in the adjoint representation, that is, we can write

$$
\left(\delta \mathcal{D}_{a b}^{\psi}\right)_{\nu}^{\nu} \equiv-\left(T^{c}\right)_{a b} \delta \mathcal{D}_{c}^{\psi} \equiv-\delta \mathcal{D}_{a b}^{\psi}
$$

and $\delta \mathcal{D}^{\psi}$ thus defined satisfies the gauge covariant equation

$$
\left[k \cdot \tilde{D}_{X}, \delta \mathcal{D}^{\psi}(k, X)\right]=i \frac{g}{2} T^{a}\left\{\bar{\psi}(X) \mathbb{K}^{a}(k, X)-\mathcal{H}^{a}(k, X) \psi(X)\right\}
$$

where $\left[\tilde{D}_{\mu}, O\right] \equiv \partial_{\mu} O+i g\left[\tilde{A}_{\mu}, O\right]$ for any $O(X) \equiv O^{a}(X) T^{a}$.

It is now straightforward to write the equation giving the current density $\mathcal{J}_{\mathrm{g}}^{\psi}$. From eqs. (3.67) and (3.70) we have

$$
\mathcal{J}_{\mathrm{g} \mu}^{\psi a}(k, X)=k_{\mu} \operatorname{Tr} T^{a} \delta \mathcal{D}^{\psi}(k, X)=N k_{\mu} \delta \mathcal{D}_{a}^{\psi}(k, X) .
$$


Then, from eq. (3.71) we deduce the following gauge covariant equation for $\mathcal{J}_{\mathrm{g} \mu}^{\psi} \equiv t^{a} \mathcal{J}_{\mathrm{g} \mu}^{\psi a}$ :

$$
\left[k \cdot D_{X}, \mathcal{J}_{\mathrm{g} \mu}^{\psi}(k, X)\right]^{a}=i \frac{g}{2} N k_{\mu}\left\{\bar{\psi}(X) \mathbb{K}^{a}(k, X)-\mathcal{H}^{a}(k, X) \psi(X)\right\} .
$$

There is, of course, a similar equation for the density $\mathcal{J}_{\mathrm{g}}^{5}$ of the axial color current induced by fermionic fields on the hard gluons.

Let us consider now the color current induced by the gauge fields, i.e. $j_{\mathrm{g}}^{A}$. Its density, $\mathcal{J}_{\mathrm{g}}^{A}$, is related to $\delta \mathcal{D}^{A}$ by eq. (3.64). From eq. (3.62), we see that $\delta \mathcal{D}_{\mu \nu}^{A a b}$ is symmetric in the Minkovski indices and belongs to the adjoint representation, that is

$$
\delta \mathcal{D}_{\mu \nu}^{A a b} \equiv\left(T^{c}\right)_{a b} \delta \mathcal{D}_{\mu \nu}^{A c}
$$

with the new functions $\delta \mathcal{D}_{\mu \nu}^{a}$ satisfying

$$
\begin{gathered}
k \cdot \partial_{X} \delta \mathcal{D}_{\mu \nu}^{A a}-\frac{g}{4} f^{a b c} A_{b}^{\rho} k^{\lambda}\left[\Gamma_{\mu \sigma \lambda \rho} \delta \mathcal{D}_{c \nu}^{A \sigma}+\Gamma_{\nu \sigma \lambda \rho} \delta \mathcal{D}_{\mu c}^{A \sigma}\right] \\
=\frac{g}{2} \Gamma_{\mu \nu \rho \lambda}\left(\partial_{X}^{\sigma} A_{a}^{\rho}\right) k^{\lambda}\left(\partial_{\sigma}^{k} \Delta\right) .
\end{gathered}
$$

In terms of these functions, the density $\mathcal{J}_{\mathrm{g}}^{A}$ is

$$
\mathcal{J}_{\mathrm{g} \mu}^{A a}(k, X)=N\left\{\delta \mathcal{D}_{\mu \nu}^{A a}(k, X) k^{\nu}-k_{\mu} \delta \mathcal{D}_{a \nu}^{A \nu}(k, X)\right\}
$$

and it depends only on the particular combinations $\delta \mathcal{D}_{\mu \nu}^{A a} k^{\nu}$ and $\delta \mathcal{D}_{a \nu}^{A \nu}$. The equations satisfied by these quantities are easily obtained from eq. (3.75). They read

$$
\begin{array}{r}
\left(k \cdot \partial_{X}\right) \delta \mathcal{D}_{\nu}^{a \nu}-g f^{a b c}\left(k \cdot A^{b}\right) \delta \mathcal{D}_{\nu}^{c \nu}+g f^{a b c} A_{b}^{\mu}\left(\delta \mathcal{D}_{\mu \nu}^{c} k^{\nu}\right) \\
=g(d-1) k_{\mu}\left(\partial_{X}^{\nu} A_{a}^{\mu}\right)\left(\partial_{\nu}^{k} \Delta\right),
\end{array}
$$

and

$$
\begin{gathered}
\left(k \cdot \partial_{X}\right)\left(\delta \mathcal{D}_{\mu \nu}^{a} k^{\nu}\right)-g f^{a b c}\left(k \cdot A^{b}\right)\left(\delta \mathcal{D}_{\mu \nu}^{c} k^{\nu}\right) \\
+\frac{g}{4} f^{a b c}\left\{k_{\mu}\left(A^{b} \cdot \delta \mathcal{D}^{c} \cdot k\right)+A_{\mu}^{b}\left(k \cdot \delta \mathcal{D}^{c} \cdot k\right)+k^{2} A_{b}^{\nu} \delta \mathcal{D}_{\nu \mu}^{c}+\left(k \cdot A^{b}\right) k^{\nu} \delta \mathcal{D}_{\nu \mu}^{c}\right\} \\
=\frac{g}{2}\left(k_{\mu} k_{\nu}-g_{\mu \nu} k^{2}\right)\left(\partial_{X}^{\rho} A_{a}^{\nu}\right)\left(\partial_{\rho}^{k} \Delta\right) .
\end{gathered}
$$

We have dropped the superscript $A$ on $\delta D$ in order to simplify the writing, and this will be done in the following equations as long as no confusion is possible. Furthermore, we have used compact notations for tensor products, such as $A^{b} \cdot \delta \mathcal{D}^{c} \cdot k \equiv A_{b}^{\rho} \delta \mathcal{D}_{\rho \nu}^{c} k^{\nu}$.

We have to solve eq. (3.78) before eq. (3.77). Indeed, its solution, $\delta \mathcal{D}_{\mu \nu}^{a} k^{\nu}$, enters the l.h.s. of eq. (3.77) for $\delta \mathcal{D}_{\nu}^{a \nu}$. In order to do so, we first replace the term proportional 
to $k^{2} \delta \mathcal{D}_{\nu \mu}^{c}$ in the l.h.s. of eq. (3.78) by the expression deduced from eq. (3.61). Second, we note that the r.h.s. of eq. (3.78) is transverse with respect to $k$, so that

$$
k^{\mu} \delta \mathcal{D}_{\mu \nu}^{a}(k, X) k^{\nu}=0 .
$$

This allows us to eliminate the corresponding term in the 1.h.s. of eq. (3.78). We are then left with the equation

$$
\begin{array}{r}
\left(k \cdot \partial_{X}\right)\left(\delta \mathcal{D}_{\mu \nu}^{a} k^{\nu}\right)-\frac{3}{4} g f^{a b c}\left(k \cdot A^{b}\right)\left(\delta \mathcal{D}_{\mu \nu}^{c} k^{\nu}\right)+\frac{g}{4} f^{a b c} k_{\mu}\left(A^{b} \cdot \delta \mathcal{D}^{c} \cdot k\right) \\
=\frac{g}{2}\left(k_{\mu} k_{\nu}-g_{\mu \nu} k^{2}\right)\left(\partial_{X}^{\rho} A_{a}^{\nu}\right)\left(\partial_{\rho}^{k} \Delta\right)-\frac{3}{4} g^{2} f^{a b c}\left(k \cdot A^{b}\right) A_{\mu}^{c} \Delta .
\end{array}
$$

The structure of this equation suggests the decomposition $\delta \mathcal{D}_{\mu \nu}^{a} k^{\nu} \equiv g A_{\mu}^{a} \Delta+\delta \mathcal{D}_{\mu}^{a}$, where $\delta \mathcal{D}_{\mu}^{a}$ are new functions which satisfy

$$
\left(k \cdot \partial_{X}\right) \delta \mathcal{D}_{\mu}^{a}-\frac{3}{4} g f^{a b c}\left(k \cdot A^{b}\right) \delta \mathcal{D}_{\mu}^{c}+\frac{g}{4} f^{a b c} k_{\mu}\left(A^{b} \cdot \delta \mathcal{D}^{c}\right)=\frac{g}{2} k_{\mu} k_{\nu}\left(\partial_{X}^{\rho} A_{a}^{\nu}\right)\left(\partial_{\rho}^{k} \Delta\right) .
$$

By comparing this equation with eq. (3.56) for the ghosts propagator, we see that $\delta \mathcal{D}_{\mu}^{a}=$ $-k_{\mu} \delta \mathcal{G}^{a}$. Therefore eq. (3.80) has the following solution

$$
\delta \mathcal{D}_{\mu \nu}^{a} k^{\nu}(k, X)=g A_{\mu}^{a}(X) \Delta(k)-k_{\mu} \delta \mathcal{G}^{a}(k, X),
$$

which, not surprisingly, relates ghost and longitudinal gluon degrees of freedom. We use this relation in order to rewrite the induced current density (3.76) as

$$
\mathcal{J}_{\mathrm{g} \mu}^{A a}(k, X)=g N \Delta(k) A_{\mu}^{a}(X)-N k_{\mu}\left(\delta \mathcal{D}_{a \nu}^{A \nu}(k, X)+\delta \mathcal{G}^{a}(k, X)\right),
$$

where the superscript $A$ has been reintroduced. The relation (3.82) can also be used to transform eq. (3.77) for $\delta \mathcal{D}_{a \nu}^{A \nu}$ into

$$
\left[k \cdot D_{X}, \delta \mathcal{D}_{\nu}^{A \nu}(k, X)\right]^{a}=g(d-1) k_{\nu}\left(\partial_{X}^{\rho} A_{a}^{\nu}\right)\left(\partial_{\rho}^{k} \Delta\right)+g f^{a b c}\left(k \cdot A^{b}\right) \delta \mathcal{G}^{c}(k, X) .
$$

We now consider the density $\mathcal{J}_{\mathrm{b}}^{A}$ of the total color current carried by gluonic degrees of freedom, $j_{\mathrm{b}}^{A}=j_{\mathrm{g}}^{A}+j_{G}+j_{\Omega}$. By adding eqs. (3.83), (3.57) and (3.17), we obtain

$$
\mathcal{J}_{\mathrm{b} \mu}^{A a}(k, X)=-g N(d-2) \Delta(k) A_{\mu}^{a}(X)-N k_{\mu}\left\{\delta \mathcal{D}_{a \nu}^{A \nu}(k, X)+2 \delta \mathcal{G}^{a}(k, X)\right\} .
$$

The first piece in the right hand side is the tadpole contribution to the induced current density, including only the $N(d-2)$ transverse gluons. The second piece contains the contributions involving the 3 -gluon vertex. In this term, the role of the ghosts is clearly seen: the term $2 \delta \mathcal{G}^{a}$ compensates for the two spurious degrees of freedom contained in $\delta \mathcal{D}_{a \nu}^{A \nu}$ 
The equation satisfied by $\mathcal{J}_{\mathrm{b}}^{A}$ follows from eqs. (3.56) and (3.84)

$$
\left[k \cdot D_{X}, \mathcal{J}_{\mathrm{b} \mu}^{A}(k, X)\right]^{a}=-g N(d-2)\left\{\left[k \cdot D_{X}, A_{\mu}\right]^{a}+k_{\mu} k_{\nu}\left(\partial_{X}^{\rho} A_{a}^{\nu}\right) \partial_{\rho}^{k}\right\} \Delta(k) .
$$

This equation is not gauge covariant, in spite of the covariant derivative in the l.h.s. and the presence in the r.h.s. of the explicit factor $N(d-2)$ indicating that only the transverse gluons contribute. This lack of covariance has its origin in the noncovariant character of the Wigner functions that we have been using. Recall, however, that the physically relevant quantity is the induced current $j_{\mathrm{b}}^{A}$, which is obtained from its density after a $k$ integration. This means that $\mathcal{J}_{\mathrm{b}}^{A}$ is determined only up to a total derivative with respect to $k$. We use this arbitrariness in order to construct a gauge covariant density. We define, in analogy with eq. (3.49),

$$
\dot{\mathcal{J}}_{\mathrm{b} \mu}^{A}(k, X) \equiv \mathcal{J}_{\mathrm{b} \mu}^{A}(k, X)+g N(d-2)\left(A \cdot \partial_{k}\right)\left(k_{\mu} \Delta(k)\right)
$$

This new density gives the same current as $\mathcal{J}_{\mathrm{b}}^{A}(k, X)$ after the $k$-integration, and it satisfies the gauge covariant equation, derived from eq. (3.86),

$$
\left[k \cdot D_{X}, \dot{\mathcal{J}}_{\mathrm{b} \mu}^{A}(k, X)\right]^{a}=g N(d-2) k_{\mu} k^{\rho} F_{\rho \nu}^{a} \partial^{\nu} \Delta(k) .
$$

We recognize here a situation similar to that encountered in Sec.3.5, in relation with $\delta \mathcal{S}_{A}$. By analogy, we can interpret eq. (3.87) as expressing the change between canonical and kinetic momenta for the $N(d-2)$ transverse gluons.

This may be seen more explicitly by expressing the current density $\mathcal{J}_{\mathrm{b}} \equiv \mathcal{J}_{\mathrm{g}}^{\psi}+\dot{\mathcal{J}}_{\mathrm{b}}^{A}$ in terms of a gauge covariant Wigner function, denoted by $\mathcal{D}(k, X)$. This is a $\left(N^{2}-1\right) \times$ $\left(N^{2}-1\right)$ color matrix whose diagonal elements may be interpreted as the distribution functions of transverse gluons of each color. In equilibrium, it reduces to

$$
\mathcal{D}_{0}^{a b}(k)=\delta^{a b}(d-2) \Delta(k),
$$

since there are $(d-2)$ polarization states for gluons of each color. Off-equilibrium,

$$
\dot{\mathcal{D}}(k, X)=\dot{\mathcal{D}}_{0}(k)+\delta \dot{\mathcal{D}}(k, X)
$$

where $\delta \mathcal{D}(k, X)$ is related to the induced current $j_{\mathrm{b}}=j_{\mathrm{b}}^{A}+j_{\mathrm{b}}^{\psi}$ by

$$
j_{\mathrm{b} \mu}^{a}(X)=g \int \frac{d^{4} k}{(2 \pi)^{4}} k_{\mu} \operatorname{tr} T^{a} \delta \mathcal{D}(k, X),
$$

with the trace acting in the color space. Since $\delta \mathcal{D}(k, X)$ belongs to the adjoint representation, it is completely determined by eq. (3.91). In line with the previous analysis, we write

$$
\delta \mathcal{D}(k, X)=\delta \dot{\mathcal{D}}_{a}^{A}(k, X) T^{a}+\delta \mathcal{D}_{a}^{\psi}(k, X) T^{a}
$$


where $\delta \mathcal{D}^{\psi}$ satisfies the gauge covariant equation (3.71), while $\delta \mathcal{D}^{A}$ obeys an equation analogous to that for $\mathcal{J}_{\mathrm{b}}^{A},(3.88$,

$$
\left[k \cdot \tilde{D}_{X}, \delta \mathcal{D}^{A}(k, X)\right]=g(d-2) k^{\rho} \tilde{F}_{\rho \nu} \partial^{\nu} \Delta(k),
$$

with $\tilde{F}_{\nu \rho} \equiv F_{\nu \rho}^{a} T^{a}$.

In Feynman gauge, the relation between $\delta \mathcal{D}^{A}(k, X)$ and the gluon and the ghost propagators is given by (see eq. (3.85))

$$
\delta \mathcal{D}^{A}(k, X)=-\left\{\delta \mathcal{D}_{\nu}^{A \nu}(k, X)+2 \delta \mathcal{G}(k, X)\right\}+g(d-2)\left(\tilde{A}(X) \cdot \partial_{k}\right) \Delta(k)
$$

If we also notice that, in the same gauge, $\mathcal{D}_{0}(k)=-D_{0 \nu}^{<\nu}(k)-2 G_{0}^{<}(k)$, we see that $\mathcal{D}(k, X)$ may be viewed as resulting from the replacement of the canonical momentum by the kinetic one in

$$
\mathcal{D}(k, X) \equiv-\left\{\mathcal{D}_{\nu}^{\nu}(k, X)+2 \mathcal{G}(k, X)\right\}
$$

That is, $\mathcal{D}(k, X) \approx \mathcal{D}(k+g \tilde{A}, X)$ in leading order, and $\mathcal{D}(k, X)$ may be interpreted as the distribution function for the transverse gluons. Of course, the separation of $\mathcal{D}(k, X)$ in terms of $\mathcal{D}_{\nu}^{\nu}$ and $\mathcal{G}$ depends upon the choice of the gauge. However, equations (3.73) and (3.88) for the induced currents, as well as the corresponding equations (3.71) and (3.93) for the covariant Wigner functions, are independent of gauge fixing, as shown in Appendix B.

\section{Kinetic equations for hard particles}

In the previous section, we have derived equations for the Wigner transforms of the offequilibrum 2-point functions which are needed in the calculation of the induced sources for the average fields. These equations describe the dynamics of the plasma particles in the presence of the average fields which may be considered at this stage as given background fields. We analyze now the structure of these equations and we write their solutions for retarded conditions, that is for average fields which vanish adiabatically as $X_{0} \rightarrow-\infty$. As we shall see, the equations for the densities of induced sources take the form of kinetic equations, that is, of equations for generalized phase space distribution functions for the plasma particles. 


\subsection{Equations for the densities of the induced sources}

The equations for the densities of the induced sources have been already written in the previous section, or can be derived in a straightforward way from the equations for the corresponding 2-point functions. Explicitly, we use eqs. (3.42), (3.50), (3.47), (3.73) and (3.88) and obtain

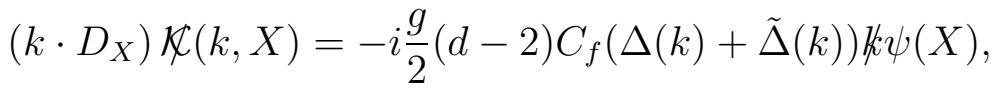

$$
\begin{aligned}
& {\left[k \cdot D_{X}, \mathcal{J}_{\mathrm{f} \mu}^{A}(k, X)\right]^{a}=2 g N_{f} k_{\mu} k^{\rho} F_{\rho \nu}^{a}(X) \partial^{\nu} \tilde{\Delta}(k),} \\
& {\left[k \cdot D_{X}, \mathcal{J}_{\mathrm{f} \mu}^{\psi}(k, X)\right]^{a}=i g k_{\mu}\left\{\bar{\psi}(X) t^{a} \not K(k, X)-\mathcal{H}(k, X) t^{a} \psi(X)\right\}} \\
& \text { - } i \frac{g}{2} N k_{\mu}\left\{\bar{\psi}(X) \mathbb{K}^{a}(k, X)-\mathcal{H}^{a}(k, X) \psi(X)\right\} \text {, } \\
& {\left[k \cdot D_{X}, \mathcal{J}_{\mathrm{b} \mu}^{\psi}(k, X)\right]^{a}=i \frac{g}{2} N k_{\mu}\left\{\bar{\psi}(X) \mathbb{K}^{a}(k, X)-\mathcal{H}^{a}(k, X) \psi(X)\right\},} \\
& {\left[k \cdot D_{X}, \dot{\mathcal{J}}_{\mathrm{b} \mu}^{A}(k, X)\right]^{a}=g N(d-2) k_{\mu} k^{\rho} F_{\rho \nu}^{a}(X) \partial^{\nu} \Delta(k),} \\
& \left(k \cdot \partial_{X}\right) \mathcal{B}_{\mu}(k, X)=i g k_{\mu}\{\bar{\psi}(X) \mathbb{K}(k, X)-\mathcal{H}(k, X) \psi(X)\}, \\
& \left(k \cdot \partial_{X}\right) \mathcal{B}_{\mu}^{5}(k, X)=i g k_{\mu}\left\{\bar{\psi}(X) \gamma_{5} \not K K(k, X)+\mathcal{H}(k, X) \gamma_{5} \psi(X)\right\} .
\end{aligned}
$$

The equations (4.1)-(4.7) are all first order partial differential equations and, to be specific, we shall solve them in this section with vanishing initial conditions at $X_{0} \rightarrow-\infty$. They describe the dynamics of the hard particles of the plasma in the presence of given background fields $A_{\mu}(X), \psi(X)$ and $\bar{\psi}(X)$ which vanish adiabatically in the remote past.

Only the real hard particles which exist in the plasma at thermal equilibrum do contribute to the induced sources. This is manifest in several ways in the previous equations. First, in the r.h.s. of eqs. (4.1)-(4.6), all possible vacuum contributions cancel. For instance, we can use the identity

$$
\tilde{\Delta}(k)+\Delta(k) \equiv \rho_{0}(k)\left[N\left(k_{0}\right)+n\left(k_{0}\right)\right]=2 \pi \delta\left(k^{2}\right)\left[N\left(\epsilon_{k}\right)+n\left(\epsilon_{k}\right)\right]
$$

where $\epsilon_{k} \equiv|\vec{k}|$, in order to see that the right hand side of eq. (4.1) vanishes at zero temperature; the zero temperature contributions to the quark and gluon propagators 
$S_{0}^{<}(k)$ and $D_{0}^{<}(k)$ are mutually compensating in their sum. Furthermore, the vacuum contribution to eqs. (4.2) and (4.5) cancels out, as $k \cdot F \cdot \partial_{k}\left[(1 / k) \delta\left(k_{0} \pm \epsilon_{k}\right)\right]=0$. Thus only thermal fluctuations contribute to $\mathbb{K}$ or $\mathcal{J}$. Second, there is a factor $(d-2)$ (eventually implicit) in the r.h.s. of all the equations involving bosonic degrees of freedom. As already dicussed, this reflects the fact that only the transverse gluons effectively contribute to these densities. Third, the equations are independent of gauge fixing, which again reflects the decoupling of the spurious degrees of freedom. This is shown explicitly in Appendix B where the equations for the 2-point functions are studied for a covariant gauge with an arbitrary gauge fixing parameter $\lambda$.

All the previous equations are covariant under local gauge transformation of the mean fields $A_{\mu}, \psi$ and $\bar{\psi}$. Therefore the induced densities determined from these equations are gauge covariant quantities. Specifically, $\mathbb{K}$ transforms like $\psi$ as vector in the fundamental representation, while $\mathcal{J}_{\mathrm{f}}^{A}, \mathcal{J}_{\mathrm{f}}^{\psi}, \mathcal{J}_{\mathrm{b}}^{\psi}$, and $\dot{\mathcal{J}}_{\mathrm{b}}^{A}$ transform like $F_{\mu \nu}$ as vectors in the adjoint representation, and $\mathcal{B}$ and $\mathcal{B}^{5}$ are scalars.

The similarity between the two equations for the vector and axial baryonic currents densities reflects the helicity conservation in the elementary interactions. The same symmetry is, of course, valid for the corresponding color currents. The densities for the axial color currents satisfy equations which can be easily obtained by inserting $\gamma_{5}$ matrices in the vector equations (4.3) and (4.4), in the same way as in going from eq. (4.6) to (4.7).

Quarks and gluons respond similarly to soft gauge and fermionic fields. This is apparent in the r.h.s. of eq. (4.1), and also in the similarity between eqs. (4.2) and (4.5). The different numerical factors in the right hand sides of these two equations simply count the relevant degrees of freedom. In fact, by adding together eqs. (4.2) and (4.5), one obtains an equation for the total density $\mathcal{J}^{A} \equiv \mathcal{J}_{\mathrm{f}}^{A}+\dot{\mathcal{J}}_{\mathrm{b}}{ }^{A}$

$$
\left[k \cdot D_{X}, \mathcal{J}_{\mu}^{A}(k, X)\right]=g k_{\mu} k \cdot F(X) \cdot \partial\left(2 N_{\mathrm{f}} \tilde{\Delta}(k)+N(d-2) \Delta(k)\right) .
$$

This equation generalizes the linearized Vlasov equation to nonabelian plasmas. Note that previous attempts to derive such an equation were based on approximation schemes mixing leading and non leading contributions in $g$ and therefore, from the present point of view, not entirely consistent [21, 22]. One can also combine eqs. (4.3) and (4.4) into a single equation for the total current density induced by the fermionic fields, $\mathcal{J}^{\psi} \equiv \mathcal{J}_{\mathrm{f}}^{\psi}+\mathcal{J}_{\mathrm{b}}^{\psi}$,

$$
\left[k \cdot D_{X}, \mathcal{J}_{\mu}^{\psi}(k, X)\right]=i g k_{\mu} t^{a}\left\{\bar{\psi}(X) t^{a} \not K(k, X)-\mathcal{H}(k, X) t^{a} \psi(X)\right\} .
$$

This equation is similar to the corresponding one in the abelian case [16]. In doing the sum of eqs. (4.3) and (4.4), the typical non-abelian effects cancel; these are contained for example in the second braces in eq. (4.3), and involve the 3-gluon vertex leading to gauge 
field insertions on the hard gluon lines. This kind of cancellation was first noted by Taylor and Wong [26] in relation with the HTL's for amplitudes involving one pair of quarks and any number of soft gluons (albeit their proof is only explicit up to three external gluons).

The right hand sides of the kinetic equations (4.1)-(4.7) contain a factor $\delta\left(k^{2}\right)$ showing that the mass shell condition for the hard particles is not affected by their interactions with the mean fields. This allows us, in the next subsection, to transform the equations for the current densities into kinetic equations for generalized distribution functions.

\subsection{Explicit solutions}

The solution of eq. (4.1) for $\mathbb{K}$ has the following general structure:

$$
\not{X}(k, X)=2 \pi \delta\left(k^{2}\right)\left\{\theta\left(k_{0}\right) \mathbb{X}^{+}(\vec{k}, X)+\theta\left(-k_{0}\right) \mathcal{X}^{-}(-\vec{k}, X)\right\} .
$$

This structure is characteristic to all the other densities that we compute below. Eq. (4.1) can be turned into equations for the functions $\mathcal{X}^{ \pm}$. It is easily verified that $\mathcal{X}^{+}=\mathcal{X}^{-} \equiv \not \mathcal{X}$, where $\mathcal{A}$ satisfies

$$
\left(v \cdot D_{X}\right) \AA(\vec{k}, X)=-i \frac{g}{2}(d-2) C_{f}\left[N\left(\epsilon_{k}\right)+n\left(\epsilon_{k}\right)\right] \psi \psi(X),
$$

with the initial condition $\not \mathcal{A}(\vec{k}, X) \rightarrow 0$ for $X_{0} \rightarrow-\infty$. Here $\psi \equiv v^{\mu} \gamma_{\mu}$, with the velocity 4-vector $v^{\mu} \equiv(1, \vec{v})$ and $\vec{v} \equiv \vec{k} / \epsilon_{k}$. This equation describes fluctuations where, under the action of a soft fermionic mean field, hard quarks are converted into hard gluons and vice versa. The quantity $\not{X}(\vec{k}, X)$ may be viewed as a generalized one body density matrix mixing fermionic and bosonic degrees of freedom. The covariant line derivative in the l.h.s. of eq. (4.12) is remininscent of the familiar drift term of elementary kinetic equations; the presence of a covariant derivative steems from the fact that $\not \mathcal{X}$ behaves as $\psi$ under gauge transformations.

The solution to eq. (4.12) can be written by introducing the parallel transporter along a straight line joining $x$ and $y$

$$
U(x, y)=P \exp \left\{-i g \int_{\gamma} d z^{\mu} A_{\mu}(z)\right\}
$$

where $\gamma$ is the path parametrized by $z^{\mu}(s)=y^{\mu}+\left(x^{\mu}-y^{\mu}\right) s$ with $0 \leq s \leq 1$, and $P$ is the path-ordering operator. Thus,

$$
U(X, X-v u)=P \exp \left\{-i g u \int_{0}^{1} d s v \cdot A(X-v u(1-s))\right\} .
$$


In order to solve eq. (4.12), we take $A_{\mu} \equiv A_{\mu}^{a} t^{a}$ in the fundamental representation. We have then

$$
\not(\vec{k}, X)=-i \frac{g}{2}(d-2) C_{f}\left[N\left(\epsilon_{k}\right)+n\left(\epsilon_{k}\right)\right] \psi \int_{0}^{\infty} d u U(X, X-v u) \psi(X-v u) .
$$

In order to verify that (4.15) is the correct solution to eq. (4.12) we may use the following formula for the line-derivative of the parallel transporter

$$
\left.\left(v \cdot D_{X}\right) U(X, Y)\right|_{Y=X-v u}=0
$$

together with the identity $\left(v \cdot \partial_{X}\right) f(X-v u)=-(\partial / \partial u) f(X-v u)$.

According to eq. (3.8), the fermionic induced source is obtained by integrating the density $\mathbb{K}(k, X)$ over $k$. By using eqs. (4.11) and (4.15), we get

$$
\eta^{i n d}(X)=-i \omega_{0}^{2} \int \frac{d \Omega}{4 \pi} \psi \int_{0}^{\infty} d u U(X, X-v u) \psi(X-v u),
$$

with $\omega_{0}^{2} \equiv(d-2) C_{f} g^{2} T^{2} / 16$.

The equation (4.10) for $\mathcal{J}^{\psi}$ can be integrated in a similar fashion. One gets

$$
\begin{aligned}
& \mathcal{J}_{\mu a}^{\psi}(k, X)=i g v_{\mu}^{\prime} \int_{0}^{\infty} d u \tilde{U}_{a b}\left(X, X-v^{\prime} u\right) \\
& \quad\left\{\bar{\psi}\left(X-v^{\prime} u\right) t^{b} \not K\left(k, X-v^{\prime} u\right)-\mathcal{H}\left(k, X-v^{\prime} u\right) t^{b} \psi\left(X-v^{\prime} u\right)\right\},
\end{aligned}
$$

where $v_{\mu}^{\prime} \equiv k_{\mu} / k_{0}=(1, \pm \vec{v})$ for $k_{0}= \pm \epsilon_{k} . \tilde{U}_{a b}\left(X, X-v^{\prime} u\right)$ is the parallel transporter in the adjoint representation, i.e., eq. (4.14) with $A_{\mu} \rightarrow \tilde{A}_{\mu} \equiv A_{\mu}^{a} T^{a}$. The function $\mathcal{H}$ has a structure similar to (4.11):

$$
\mathcal{H}(k, X)=2 \pi \delta\left(k^{2}\right)\left\{\theta\left(k_{0}\right) \overline{\mathcal{X}}(\vec{k}, X)+\theta\left(-k_{0}\right) \overline{\mathcal{X}}(-\vec{k}, X)\right\}
$$

where $\overline{\mathcal{A}}(\vec{k}, X) \equiv \mathcal{L}^{\dagger}(\vec{k}, X) \gamma^{0}$.

By integrating eq. (4.18) over $k$ in order to get the induced current $j_{\mu}^{\psi}(X)$, we obtain, after simple manipulations,

$$
j_{\mu}^{\psi a}(X)=g \int \frac{d^{3} k}{(2 \pi)^{3}} v_{\mu}\left[\delta n_{+}^{\psi a}(\vec{k}, X)-\delta n_{-}^{\psi a}(\vec{k}, X)\right]
$$

where

$$
\begin{aligned}
& \delta n_{ \pm}^{\psi a}(\vec{k}, X)= \pm i \frac{g}{2 \epsilon_{k}} \int_{0}^{\infty} d u \tilde{U}_{a b}(X, X-v u) \\
& \left\{\bar{\psi}(X-v u) t^{b} \not(k, X-v u)-\bar{X}(k, X-v u) t^{b} \psi(X-v u)\right\}
\end{aligned}
$$


are fluctuations in the quark and antiquark color densities induced by the fermionic mean fields. By replacing $\not \mathcal{X}$ by its explicit expression (4.15), we can evaluate the integral over $k \equiv \epsilon_{k}$ in $(4.20)$ and get

$$
\begin{aligned}
j_{\mu}^{\psi}(X)= & g \omega_{0}^{2} t^{a} \int \frac{d \Omega}{4 \pi} v_{\mu} \int_{0}^{\infty} d u \int_{0}^{\infty} d u^{\prime} \tilde{U}_{a b}(X, X-v u) \\
& \left\{\bar{\psi}(X-v u) t^{b} \psi U\left(X-v u, X-v\left(u+u^{\prime}\right)\right) \psi\left(X-v\left(u+u^{\prime}\right)\right)\right. \\
& \left.+\bar{\psi}\left(X-v\left(u+u^{\prime}\right)\right) \psi U\left(X-v\left(u+u^{\prime}\right), X-v u\right) t^{b} \psi(X-v u)\right\} .
\end{aligned}
$$

We recognize in this expression the two terms which correspond to the two possible time orderings of the scattering processes between the hard particles and the soft fermionic fields. One can obtain a more compact expression by performing simple changes of variables. Setting $s=u+u^{\prime}$ and $t=u$ one transforms the first integrals over $u, u^{\prime}$ into

$$
\int_{0}^{\infty} d t \int_{t}^{\infty} d s \tilde{U}_{a b}(X, X-v t) \bar{\psi}(X-v t) \psi t^{b} U(X-v t, X-v s) \psi(X-v s) .
$$

In the second integrals, we set $s=u$ and $t=u+u^{\prime}$, and obtain

$$
\int_{0}^{\infty} d t \int_{0}^{t} d s \tilde{U}_{a b}(X, X-v t) \bar{\psi}(X-v t) \psi U(X-v t, X-v s) t^{b} \psi(X-v s) .
$$

By using the identity

$$
\tilde{U}_{a b}(X, X-v t) t^{b}=U(X-v t, X) t^{a} U(X, X-v t),
$$

together with the group property of the parallel transporter,

$$
U(X-v t, X-v s)=U(X-v t, X) U(X, X-v s),
$$

we finally obtain

$$
\begin{aligned}
j_{\mu}^{\psi}(X)=g \omega_{0}^{2} t^{a} \int \frac{d \Omega}{4 \pi} v_{\mu} \int_{0}^{\infty} d t \int_{0}^{\infty} d s \\
\bar{\psi}(X-v t) \psi U(X-v t, X) t^{a} U(X, X-v s) \psi(X-v s) .
\end{aligned}
$$

We consider now the equations (4.6) and (4.7) for the baryonic currents. The solution to eq. (4.6) is of the form

$$
\mathcal{B}_{\mu}(k, X)=2 k_{\mu}\left(2 \pi \delta\left(k^{2}\right)\right)\left\{\theta\left(k^{0}\right) \delta n_{+}(\vec{k}, X)+\theta\left(-k^{0}\right) \delta n_{-}(-\vec{k}, X)\right\},
$$

where $\delta n_{+}(\vec{k}, X)$ and $\delta n_{-}(\vec{k}, X)$ are (color singlet) fluctuations in the quark and antiquark number densities. These satisfy

$$
\left(v \cdot \partial_{X}\right) \delta n_{ \pm}(\vec{k}, X)= \pm i \frac{g}{2 \epsilon_{k}}[\bar{\psi}(X) \not \mathcal{A}(\vec{k}, X)-\bar{A}(\vec{k}, X) \psi(X)] .
$$


This equation is solved similarly to the previous one for $\mathcal{J}^{\psi}$ (see eq. (4.21)). Then we can calculate the baryonic current

$$
b_{\mu}(X)=\int \frac{d^{4} k}{(2 \pi)^{4}} \mathcal{B}_{\mu}(k, X)=\int \frac{d^{3} k}{(2 \pi)^{3}} v_{\mu}\left[\delta n_{+}(\vec{k}, X)-\delta n_{-}(\vec{k}, X)\right],
$$

and obtain

$$
\begin{aligned}
b_{\mu}(X)=g \omega_{0}^{2} \int \frac{d \Omega}{4 \pi} v_{\mu} \int_{0}^{\infty} d t \int_{0}^{\infty} d s \\
\bar{\psi}(X-v t) \psi U(X-v t, X-v s) \psi(X-v s) .
\end{aligned}
$$

This is, of course, similar to $j_{\mu}^{\psi}$, eq. (4.27), but without the color matrices $t^{a}$. In a complete analoguous way, we also obtain the axial baryonic current

$$
\begin{aligned}
b_{\mu}^{5}(X)=g \omega_{0}^{2} \int \frac{d \Omega}{4 \pi} v_{\mu} \int_{0}^{\infty} d t \int_{0}^{\infty} d s \\
\bar{\psi}(X-v t) \gamma_{5} \psi U(X-v t, X-v s) \psi(X-v s) .
\end{aligned}
$$

We turn now to the color currents induced by soft gauge fields. Eq. (4.2) for $\mathcal{J}_{\mathrm{f}}^{A}$ implies

$$
\mathcal{J}_{\mathrm{f} \mu}^{A}(k, X)=2 k_{\mu} N_{f} t^{a}\left(2 \pi \delta\left(k^{2}\right)\right)\left\{\theta\left(k^{0}\right) \delta n_{+}^{A a}(\vec{k}, X)+\theta\left(-k^{0}\right) \delta n_{-}^{A a}(-\vec{k}, X)\right\},
$$

where $\delta n_{ \pm}^{A} \equiv \delta n_{ \pm}^{A a} t^{a}$ are fluctuations in the quark color densities. These fluctuations satisfy (with $\epsilon_{k} \equiv|\vec{k}|$ )

$$
\left[v \cdot D_{X}, \delta n_{ \pm}^{A}(\vec{k}, X)\right]=\mp g \vec{v} \cdot \vec{E}(X) \frac{d n\left(\epsilon_{k}\right)}{d \epsilon_{k}} .
$$

Here $\vec{E}(X) \equiv \vec{E}^{a}(X) t^{a}$ is the average chromoelectric field, $E_{a}^{i} \equiv F_{a}^{i 0}$; possible chromomagnetic contributions cancel because the equilibrium distribution $n\left(\epsilon_{k}\right)$ is isotropic. In the abelian case, this equation coincides with the linearized Vlasov equation. Here, the gauge field not only modifies the motion of the particle, through the color electric field that it generates, but it also induces a "precession" of the densities in color space, through the covariant derivative on the l.h.s. The solution of eq. (4.34) is

$$
\delta n_{ \pm}^{A}(\vec{k}, X)=\mp g \int_{0}^{\infty} d u U(X, X-v u) \vec{v} \cdot \vec{E}(X-v u) U(X-v u, X) \frac{d n\left(\epsilon_{k}\right)}{d \epsilon_{k}}
$$

Similarly, eqs. (4.5) or (3.93) describe oscillations in the gluon color densities $\delta N^{A} \equiv$ $\delta N_{a}^{A} t^{a}$. They satisfy an equation similar to (4.34), viz.

$$
\left[v \cdot D_{X}, \delta N^{A}(\vec{k}, X)\right]=-g \vec{v} \cdot \vec{E}(X) \frac{d N\left(\epsilon_{k}\right)}{d \epsilon_{k}} .
$$


The total induced current $j^{A}=j_{\mathrm{f}}^{A}+j_{\mathrm{b}}^{A}$ can be expressed in terms of these color density fluctuations. Thus

$$
j_{\mathrm{f} \mu}^{A}(X)=g N_{\mathrm{f}} \int \frac{d^{3} k}{(2 \pi)^{3}} v_{\mu}\left[\delta n_{+}^{A}(\vec{k}, X)-\delta n_{-}^{A}(\vec{k}, X)\right],
$$

and

$$
j_{\mathrm{b} \mu}^{A}(X)=g(d-2) N \int \frac{d^{3} k}{(2 \pi)^{3}} v_{\mu} \delta N^{A}(\vec{k}, X) .
$$

Then, by using eq. (4.35) for $\delta n_{ \pm}^{A}(\vec{k}, X)$ and a similar expression for $\delta N^{A}(\vec{k}, X)$, we obtain

$$
j_{\mu}^{A}(X)=3 \omega_{p}^{2} \int \frac{d \Omega}{4 \pi} v_{\mu} \int_{0}^{\infty} d u U(X, X-v u) F_{0 j}(X-v u) v^{j} U(X-v u, X),
$$

where $\omega_{p}^{2} \equiv\left((d-2) N+N_{\mathrm{f}}\right) g^{2} T^{2} / 18$ is the plasma frequency.

As a last remark, we note that the final expressions for the induced sources contain no explicit reference to the nature of the hard particles involved. This information is all contained in the explicit expressions of the two frequencies $\omega_{0}$ and $\omega_{p}$, which are obtained as simple momentum integrals involving the statistical occupation factors $N(k)$ and $n(k)$. These expressions may be easily generalized to the case of systems with a non vanishing chemical potential $\mu$ :

$$
\omega_{0}^{2}=g^{2} C_{f} \frac{d-2}{16}\left(T^{2}+\frac{\mu^{2}}{\pi^{2}}\right), \quad \omega_{p}^{2}=g^{2}\left\{\frac{1}{18}\left((d-2) N+N_{f}\right) T^{2}+\frac{N_{f}}{6} \frac{\mu^{2}}{\pi^{2}}\right\} .
$$

\section{$5 \quad$ Mean fields dynamics}

In Sec.3, we have derived consistent equations of motion for the 2-point functions which describe, at leading order in $g$, the response of the plasma to weak and slowly varying fields. By solving these equations in Sec.4, we have expressed the induced sources, which enter the mean fields equations, in terms of the average fields themselves, thereby closing the system which determines the dynamics of the mean fields.

The mean field equations,

$$
i \not D \psi(X)=\eta(X)+\eta^{i n d}(X)
$$


and

$$
\left[D^{\nu}, F_{\nu \mu}(X)\right]^{a}-g \bar{\psi}(X) \gamma_{\mu} t^{a} \psi(X)=j_{\mu}^{a}(X)+j_{\mu}^{i n d a}(X)
$$

have a few remarkable properties, which are worth recalling.

(a) As shown in Sec.4.1, the induced sources are covariant under local gauge transformations of the mean fields. Specifically, $\eta^{\text {ind }}$ transforms like $\psi$, while $j^{\text {ind }}$ transforms as $F_{\mu \nu}^{a}$. It follows that eqs. (5.1) and (5.2) are gauge covariant if the external sources transform covariantly. This covariance is that of the Euler-Lagrange equations of motion deduced from the classical QCD action, (i.e., the tree level action, eq. (2.4), but without the ghosts and the gauge fixing terms). It is noteworthy to recover gauge covariance at the level of eqs. (5.1)-(5.2), where corrections to the tree level dynamics are taken into account through the induced sources.

(b) The derivatives of the mean fields in the 1.h.s. of eqs. (5.1) and (5.2) are of the same order of magnitude as the induced sources in the r.h.s. That is, $\eta^{\text {ind }} \sim$ $g T \psi \sim \partial_{X} \psi$ and $j^{A} \sim g T F \sim \partial_{X} F$, as can be readily verified on the explicit expressions given in eqs. (4.17) and (4.39). Thus, the propagation of soft modes is nonperturbatively renormalized by the medium, reflecting the collective character of the long wavelength excitations.

(c) For average fields whith maximum strength, i.e., $F \sim g T^{2}$ and $\bar{\psi} \psi \sim g T^{3}$, all the terms appearing in eqs. (5.1)-(5.2) are of the same order of magnitude. The equations are then nonlinear, due to the covariant derivatives in their l.h.s., as well as those which enter the structure of the induced sources. This is the only source of nonlinearity, showing that the limits on the fields strength we are using are equivalent to neglecting all genuine non linear dynamical effects of the fields.

(d) Eqs. (5.1) and (5.2) are nonlocal, which is a manifestation of the fact that the ultrarelativistic QCD plasma is a dispersive polarizable medium.

For vanishing external sources, the mean field equations describe the normal modes of the plasma, or equivalently its soft quasiparticles. These equations have been solved only in the case where the gauge fields are weak, that is in the linear, abelian, regime. Then the oscillations of the fermion and gauge fields decouple, and explicit dispersion relations can be obtained [14, 15, 33, 34]. In general, quark and gluon modes mix, and the non linearity of the equations make them difficult to solve. Leaving aside this difficult problem, we note that the mean field equations summarize a lot of information about the soft quasiparticles, such as their effective propagators, and the effective vertices which describe their mutual interactions. We show in the next subsections how these can be 
obtained from the induced sources, and derive the simple Ward identities that they satisfy.

\subsection{Hard thermal loops from induced sources}

As pointed out in Sec.2.2, the knowledge of the induced sources as functionals of the fields allows us to derive, by functional differentiation, the one particle irreducible amplitudes for the corresponding fields. We use this procedure now, in order to construct self-energies and proper vertices for soft quasiparticles valid at leading order in $g$. In doing this, we use boundary conditions such that the mean fields vanish at $X_{0} \rightarrow-\infty$, as in the previous section. The resulting amplitudes will be causal with respect to the time argument in the induced source.

In order to proceed in a systematic way, it is convenient to introduce retarded and advanced Green's functions for the covariant line derivative $v \cdot D_{X}$, denoted as $G_{r e t}(X, Y ; v)$ and $G_{a d v}(X, Y ; v)$, respectively. Thus, $G_{r e t}(X, Y ; v)$ is defined by

$$
i\left(v \cdot D_{X}\right) G_{r e t}(X, Y ; v)=\delta^{(4)}(X-Y), \quad G_{r e t}(X, Y ; v)=0 \text { for } X_{0}<Y_{0},
$$

and $G_{a d v}(X, Y ; v)$ satisfies a similar equation, but $G_{a d v}(X, Y ; v)=0$ for $X_{0}>Y_{0}$. The solution to eq. (5.3) may be written as

$$
\begin{aligned}
G_{r e t}(X, Y ; v) & =-i \theta\left(X^{0}-Y^{0}\right) \delta^{(3)}\left(\vec{X}-\vec{Y}-\vec{v}\left(X^{0}-Y^{0}\right)\right) U(X, Y) \\
& =-i \int_{0}^{\infty} d u \delta^{(4)}(X-Y-v u) U(X, Y),
\end{aligned}
$$

where $U(X, Y)$ is the parallel transporter (4.13). The use of this Green's function is appropriate for the study of the response of the plasma to perturbations which vanish as $X_{0} \rightarrow-\infty$. One could also consider perturbations which vanish adiabatically when $X_{0} \rightarrow \infty$. Then, the induced currents would be expressed in terms of the advanced Green's function,

$$
G_{a d v}(X, Y ; v)=i \int_{0}^{\infty} d u \delta^{(4)}(X-Y+v u) U(X, Y),
$$

which is related to $G_{r e t}$ by $G_{a d v}(X, Y ; v)=G_{r e t}^{\dagger}(Y, X ; v)$, where the Hermitian conjugaison refers to color indices. For $A_{\mu}^{a}=0$, the Fourier components of $G_{r e t}$ an $G_{a d v}$ are simply $G_{r e t}(P)=(v \cdot P+i \eta)^{-1}=G_{a d v}^{*}(P)$, with $\eta \rightarrow 0^{+}$.

According to equations like (2.27) or (2.30), $\eta^{\text {ind }}$ acts as a generating functional for all the proper vertices having at least one pair of external fermion lines and any number $N$ of bosonic lines. In the present approximation, $\eta^{\text {ind }}$ is given by eq. (4.17), which can 
also be written as

$$
\eta^{i n d}(X) \equiv \int d^{4} Y \delta \Sigma^{A}(X, Y) \psi(Y)
$$

Since $\eta^{\text {ind }}$ is linear in $\psi$, the only amplitudes which do not vanish at the level of the present approximation involve in fact no more than one pair of external fermions. The kernel $\delta \Sigma^{A}$ may be identified with the self energy of a soft fermion propagating in a background gauge field (see eq. (2.27)). In order to get its explicit form, we rewrite eq. (4.17) for $\eta^{\text {ind }}$ as

$$
\eta^{i n d}(X)=\omega_{0}^{2} \int \frac{d \Omega}{4 \pi} \psi \int d^{4} Y G_{r e t}(X, Y ; v) \psi(Y) .
$$

Then, obviously,

$$
\delta \Sigma^{A}(X, Y)=\omega_{0}^{2} \int \frac{d \Omega}{4 \pi} \psi G_{r e t}(X, Y ; v)
$$

which is similar to the corresponding abelian expression [16]. The only manifestation of the underlying gauge group is the presence of the color factor $C_{f}$ in the expression of $\omega_{0}$, together with the non-trivial color structure of $G_{r e t}$. Eq. (5.4) implies $\delta \Sigma^{A}(X, Y)=0$ for $X^{0}<Y^{0}$, reflecting the retarded boundary conditions in eq. (5.7) for $\eta^{\text {ind }}(X)$. For $A=0$, eq. (5.8) gives the well-known HTL expression for the soft quark (retarded) self energy

$$
\delta \Sigma(P)=\omega_{0}^{2} \int \frac{d \Omega}{4 \pi} \frac{\psi}{v \cdot P+i \eta} .
$$

Note that, because $P \sim g T$, the correction (5.9) to the quark inverse propagator is of the same order of magnitude as the tree-level amplitude $\left(S_{0}^{-1}(P) \sim \not P \sim g T\right)$. It can be used to construct the equilibrium propagator for a soft quark, correct at leading order in $g$ : ${ }^{*} S^{-1}(P)=-\not P+\delta \Sigma(P)[2]$.

Because eq. (5.8) is valid for arbitrary (soft) background gauge fields, we can use it to derive - by successive functional differentiations - expressions for all amplitudes involving one fermion pair and any number of soft gluons. To be specific, we define the correction to the amplitude between a quark pair and $N$ soft gluons by

$$
g^{N} \delta \Gamma_{\mu_{1} \ldots \mu_{N}}^{a_{1} \ldots a_{N}}\left(X_{1}, \ldots, X_{N} ; Y_{1}, Y_{2}\right)=\frac{\delta^{N}}{\delta A_{a_{N}}^{\mu_{N}}\left(X_{N}\right) \ldots \delta A_{a_{1}}^{\mu_{1}}\left(X_{1}\right)}\left(\delta \Sigma^{A}\left(Y_{1}, Y_{2}\right)\right)
$$

In doing these differentiations, we use identities like

$$
\frac{\delta G_{r e t}\left(X_{1}, X_{2} ; v\right)}{\delta A_{a}^{\mu}(Y)}=g v_{\mu} G_{r e t}\left(X_{1}, Y ; v\right) t^{a} G_{r e t}\left(Y, X_{2} ; v\right),
$$

and similarly for $G_{a d v}$, which follow from eqs. (5.4) and (4.13). The normalization we choose for the amplitudes (5.10) is such that $\delta \Gamma$ depends on $g$ only through $\omega_{0}^{2}$. For 
all these amplitudes, $Y_{1}^{0}$ is the largest time, while $Y_{2}^{0}$ is the smallest one. The relative chronological ordering of the $N$ gluon lines is arbitrary, and, in fact, the amplitudes are totally symmetric under their permutations. Up to minor changes due to the color algebra, all the amplitudes obtained in this way coincide with the corresponding abelian amplitudes [16].

For the sake of illustration, we give now the explicit expressions for the amplitudes involving a quark pair and one or two gluons. The first case corresponds to the quarkgluon vertex correction in the presence of a background gauge field $A_{\mu}$ (see eq. (2.30)

$$
\delta \Gamma_{\mu}^{A a}\left(X ; Y_{1}, Y_{2}\right)=\omega_{0}^{2} \gamma^{\nu} \int \frac{d \Omega}{4 \pi} v_{\mu} v_{\nu} G_{r e t}\left(Y_{1}, X ; v\right) t^{a} G_{r e t}\left(X, Y_{2} ; v\right)
$$

where the time arguments satisfy $Y_{1}^{0} \geq X^{0} \geq Y_{2}^{0}$. For $A=0$, eq. (5.12) gives the HTL correction to the quark-gluon vertex [3], with appropriate boundary conditions. Defining the Fourier transform of $\delta \Gamma_{\mu}^{a}$ by

$$
\begin{aligned}
& (2 \pi)^{4} \delta^{(4)}\left(P+K_{1}+K_{2}\right) \delta \Gamma_{\mu}^{a}\left(P ; K_{1}, K_{2}\right) \equiv \\
& \quad \int d^{4} X d^{4} Y_{1} d^{4} Y_{2} e^{i\left(P \cdot X+K_{1} \cdot Y_{1}+K_{2} \cdot Y_{2}\right)} \delta \Gamma_{\mu}^{a}\left(X ; Y_{1}, Y_{2}\right),
\end{aligned}
$$

we get

$$
\delta \Gamma_{\mu}^{a}\left(P ; K_{1}, K_{2}\right)=-t^{a} \omega_{0}^{2} \gamma^{\nu} \int \frac{d \Omega}{4 \pi} \frac{v_{\mu} v_{\nu}}{\left(v \cdot K_{1}+i \eta\right)\left(v \cdot K_{2}-i \eta\right)} \equiv t^{a} \delta \Gamma_{\mu}\left(P ; K_{1}, K_{2}\right) .
$$

As the external momenta are all of order $g T, g \delta \Gamma_{\mu}$ is of the same order as the bare vertex $g \gamma_{\mu}$. Thus, the effective quark-gluon vertex at leading order in $g$ is $g t^{a *} \Gamma_{\mu}$, where ${ }^{*} \Gamma_{\mu} \equiv \gamma_{\mu}+\delta \Gamma_{\mu}[2]$.

The second example is that of the vertex between a quark pair and two gluons, $g^{2 *} \Gamma_{\mu \nu}^{a b}$. This vertex does not exist at tree level, and in leading order it arises entirely from the hard thermal loop, ${ }^{*} \Gamma_{\mu \nu}^{a b}=\delta \Gamma_{\mu \nu}^{a b}$, with 


$$
\begin{aligned}
\delta \Gamma_{\mu \nu}^{a b}\left(X_{1}, X_{2} ; Y_{1}, Y_{2}\right)= & \omega_{0}^{2} \gamma^{\rho} \int \frac{d \Omega}{4 \pi} v_{\mu} v_{\nu} v_{\rho} \\
& \left\{G_{r e t}\left(Y_{1}, X_{1} ; v\right) t^{a} G_{r e t}\left(X_{1}, X_{2} ; v\right) t^{b} G_{r e t}\left(X_{2}, Y_{2} ; v\right)\right. \\
& \left.+G_{r e t}\left(Y_{1}, X_{2} ; v\right) t^{b} G_{r e t}\left(X_{2}, X_{1} ; v\right) t^{a} G_{r e t}\left(X_{1}, Y_{2} ; v\right)\right\} .
\end{aligned}
$$

For $A=0$, defining the Fourier transform as in eq. (5.13), we obtain

$$
\begin{aligned}
\delta \Gamma_{\mu \nu}^{a b}\left(P_{1}, P_{2} ; K_{1}, K_{2}\right)= & -\omega_{0}^{2} \gamma^{\rho} \int \frac{d \Omega}{4 \pi} \frac{v_{\mu} v_{\nu} v_{\rho}}{\left(v \cdot K_{1}+i \eta\right)\left(v \cdot K_{2}-i \eta\right)} \\
& \left\{\frac{t^{a} t^{b}}{v \cdot\left(K_{1}+P_{1}\right)+i \eta}+\frac{t^{b} t^{a}}{v \cdot\left(K_{1}+P_{2}\right)+i \eta}\right\},
\end{aligned}
$$

which, when summed over the color indices, coincides with the expressions given in refs. [2, 8].

Alternatively, we can derive the amplitudes (5.10) from the expression (4.27) for the induced current $j^{\psi}(X) \equiv j_{\mathrm{f}}^{\psi}(X)+j_{\mathrm{b}}^{\psi}(X)$, but, this time, the boundary conditions will be different, as the time argument of $j^{\psi}(X)$ is now the largest one. We first use the definitions (5.4) and (5.5) to rewrite $j^{\psi}(X)$, eq. (4.27), as

$$
\begin{aligned}
j_{\mu}^{\psi}(X)= & g t^{a} \omega_{0}^{2} \int \frac{d \Omega}{4 \pi} v_{\mu} \int d^{4} Y_{1} d^{4} Y_{2} \\
& \bar{\psi}\left(Y_{1}\right) \psi G_{a d v}\left(Y_{1}, X ; v\right) t^{a} G_{r e t}\left(X, Y_{2} ; v\right) \psi\left(Y_{2}\right) .
\end{aligned}
$$

The resulting correction to the quark-gluon vertex is (recall the second equality in eq. (2.30))

$$
\delta \Gamma_{\mu}^{A a}\left(X ; Y_{1}, Y_{2}\right)=\omega_{0}^{2} \gamma^{\nu} \int \frac{d \Omega}{4 \pi} v_{\mu} v_{\nu} G_{a d v}\left(Y_{1}, X ; v\right) t^{a} G_{r e t}\left(X, Y_{2} ; v\right),
$$

where now the time arguments satisfy $X^{0} \geq \max \left(Y_{1}^{0}, Y_{2}^{0}\right)$, the chronological order of $Y_{1}$ and $Y_{2}$ being arbitrary (compare, in this respect, with eq. (5.12) above). For $A=0$, we have

$$
\delta \Gamma_{\mu}^{a}\left(P ; K_{1}, K_{2}\right)=-t^{a} \omega_{0}^{2} \gamma^{\nu} \int \frac{d \Omega}{4 \pi} \frac{v_{\mu} v_{\nu}}{\left(v \cdot K_{1}-i \eta\right)\left(v \cdot K_{2}-i \eta\right)},
$$

which differs from (5.14) solely by the $i \eta$ 's in the denominators reflecting the respective boundary conditions. If we further differentiate the $\delta \Gamma_{\mu}^{A a}\left(X_{1} ; Y_{1}, Y_{2}\right)$ of eq. (5.18) with respect to $A_{\mu}$, we generate amplitudes of the type (5.10), in which $X_{1}^{0}$ is the largest time. 
All the amplitudes involving only soft gluons may be derived from the induced current $j^{A}$. In the present approximation, $j^{A}$ is given by eq. (4.39), which, after some algebraic manipulations, can be cast into the form

$$
j_{\mu}^{A a}(X)=-3 \omega_{p}^{2} A_{0}^{a}(X) g_{0 \mu}+3 i \omega_{p}^{2} \int \frac{d \Omega}{4 \pi} v_{\mu} \int d^{4} Y \tilde{G}_{r e t}^{a b}(X, Y)\left(v \cdot \dot{A}^{b}(Y)\right),
$$

where $\tilde{G}_{r e t}$ is the retarded Green's function (5.4) written in the adjoint representation (i.e., with $U \rightarrow \tilde{U}$ ), while $\dot{A} \equiv \partial_{0} A$. By successive differentiations with respect to $A$ in $j_{\mu}^{A}(X)$, eq. (5.20), we derive equilibrium amplitudes with any number of soft gluon external lines, which are causal with respect to $X_{0}$. For example, by using eq. (2.26) one gets, after a simple calculation, the well-known expression of the (retarded) soft gluon polarization tensor:

$$
\delta \Pi_{\mu \nu}^{a b}(P)=3 \omega_{p}^{2} \delta^{a b}\left\{-\delta_{\mu}^{0} \delta_{\nu}^{0}+P^{0} \int \frac{d \Omega}{4 \pi} \frac{v_{\mu} v_{\nu}}{v \cdot P+i \eta}\right\} .
$$

As $P \sim g T$, this is of the same order as the tree-level inverse propagator, $D_{0}^{-1}(P) \sim$ $P^{2} \sim g^{2} T^{2}$. Thus, in leading order, the effective propagator for soft gluons is ${ }^{*} D_{\mu \nu}^{-1}(P) \equiv$ $D_{0 \mu \nu}^{-1}(P)+\delta \Pi_{\mu \nu}(P)$ [1, 2]. Note that the constant term in eq. (5.21) stems from the first term (linear in $A_{0}$ ) of the expression eq. (5.20) for $j^{A}$; this term does not contribute to amplitudes with $N \geq 3$ external lines. As a further example, we write the expression for the three-gluon vertex correction which follows by a second differentiation with respect to $A$ (as in eq. (2.31)). Using the same definition as in (5.13) for the Fourier transform, we obtain from eq. (5.20)

$$
\begin{aligned}
\delta \Gamma_{\mu \nu \rho}^{a b c}\left(P_{1}, P_{2}, P_{3}\right)= & \text { if }{ }^{a b c} 3 \omega_{p}^{2} \int \frac{d \Omega}{4 \pi} \frac{v_{\mu} v_{\nu} v_{\rho}}{v \cdot P_{1}+i \eta} \\
& \left\{\frac{P_{3}^{0}}{v \cdot P_{3}-i \eta}-\frac{P_{2}^{0}}{v \cdot P_{2}-i \eta}\right\},
\end{aligned}
$$

where the imaginary parts in the denominators correspond to the time orderings $X_{1}^{0} \geq$ $X_{2}^{0} \geq X_{3}^{0}$ for the first term inside the parantheses, and, respectively, $X_{1}^{0} \geq X_{3}^{0} \geq X_{2}^{0}$ for the second term. We can rewrite this more symmetrically as $\delta \Gamma_{\mu \nu \rho}^{a b c} \equiv i f^{a b c} \delta \Gamma_{\mu \nu \rho}$ with [3]

$$
\begin{gathered}
\delta \Gamma_{\mu \nu \rho}\left(P_{1}, P_{2}, P_{3}\right)=\omega_{p}^{2} \int \frac{d \Omega}{4 \pi} v_{\mu} v_{\nu} v_{\rho}\left\{\frac{P_{1}^{0}-P_{2}^{0}}{\left(v \cdot P_{1}+i \eta\right)\left(v \cdot P_{2}-i \eta\right)}\right. \\
\left.+\frac{P_{2}^{0}-P_{3}^{0}}{\left(v \cdot P_{2}-i \eta\right)\left(v \cdot P_{3}-i \eta\right)}+\frac{P_{3}^{0}-P_{1}^{0}}{\left(v \cdot P_{3}-i \eta\right)\left(v \cdot P_{1}+i \eta\right)}\right\} .
\end{gathered}
$$

Generally, all the amplitudes with soft external gluons which are derived in this way coincide with the corresponding HTL's of the diagrammatic approach [3, 2], with boundary conditions such that $X_{1}^{0}$ is the largest time, while all the other external lines enter symmetrically. 


\subsection{Conservation laws and Ward identities}

The induced currents satisfy covariant conservation laws related to the symmetries of the QCD lagrangian. These can be easily derived from the equations of motion for the corresponding densities. These conservation laws imply, in particular, simple Ward identities relating the hard thermal loops.

By acting on eq. (4.10) with the covariant derivative $D_{X}^{\mu}$, we get

$$
\left[D_{X}^{\mu},\left[k \cdot D_{X}, \mathcal{J}_{\mu}^{\psi}(k, X)\right]\right]=\left[k \cdot D_{X}, i g t^{a}\left(\bar{\psi}(X) t^{a} \not \mathbb{K}(k, X)-\mathcal{H}(k, X) t^{a} \psi(X)\right)\right] .
$$

In the left hand side, we use the Jacobi identity to write

$$
\left[D_{X}^{\mu},\left[k \cdot D_{X}, \mathcal{J}_{\mu}^{\psi}\right]\right]=\left[k \cdot D_{X},\left[D_{X}^{\mu}, \mathcal{J}_{\mu}^{\psi}\right]\right]-i g\left[\mathcal{J}_{\mu}^{\psi}, F^{\mu \nu} k_{\nu}\right]
$$

where the definition of the field tensor $i g F_{\mu \nu} \equiv\left[D_{\mu}, D_{\nu}\right]$ has been used. Since $\mathcal{J}_{\mu}^{\psi}$ is proportional to $k_{\mu}$, as shown by eq. (4.10), the last term cancels in the right hand side of eq. (5.25). Then eq. (5.24) leads to

$$
\left[D_{X}^{\mu}, \mathcal{J}_{\mu}^{\psi}(k, X)\right]=i g t^{a}\left(\bar{\psi}(X) t^{a} \not K(k, X)-\mathcal{H}(k, X) t^{a} \psi(X)\right) .
$$

After integrating over $k$, this becomes a continuity equation for $j^{\psi}$ :

$$
\left[D^{\mu}, j_{\mu}^{\psi}(X)\right]=i g t^{a}\left(\bar{\psi}(X) t^{a} \eta^{i n d}(X)-\bar{\eta}^{i n d}(X) t^{a} \psi(X)\right) .
$$

Furthermore, the equations of motion for the fermionic fields $\psi$ and $\bar{\psi}$, that is, eq. (5.1) and its Hermitian conjugate, may be used to show that

$$
\left[D^{\mu}, g t^{a} \bar{\psi} \gamma_{\mu} t^{a} \psi\right]=-i g t^{a}\left(\bar{\psi} t^{a} \eta-\bar{\eta} t^{a} \psi\right)-i g t^{a}\left(\bar{\psi} t^{a} \eta^{i n d}-\bar{\eta}^{i n d} t^{a} \psi\right) .
$$

By adding eqs. (5.27) and (5.28), we finally obtain the continuity equation satisfied by the total current $g \bar{\psi} \gamma_{\mu} t^{a} \psi+j_{\mu}^{\psi a}$ associated to the fermionic mean fields:

$$
\left[D^{\mu}, g t^{a} \bar{\psi} \gamma_{\mu} t^{a} \psi+j_{\mu}^{\psi}\right]=-i g t^{a}\left(\bar{\psi} t^{a} \eta-\bar{\eta} t^{a} \psi\right)
$$

Thus the current $g \bar{\psi} \gamma_{\mu} t^{a} \psi+j_{\mu}^{\psi a}$ is conserved in the absence of external fermionic sources.

Similar conservation laws hold for the axial color current and for the baryonic currents $(4.31$ ) and 4.32 ).

Repeating the same steps as above, but starting with the Vlasov equation (4.9), we obtain the covariant conservation law for the induced current $j^{A}$ :

$$
\left[D^{\mu}, j_{\mu}^{A}(X)\right]=0
$$


Finally, by using the conservation laws for the induced currents, eqs. (5.29)-(5.30), together with the Jacobi identity $\left[D^{\mu},\left[D^{\nu}, F_{\nu \mu}\right]\right]=0$, we see that, in order for the mean fields equations (5.1) and (5.2) to be consistent, the external sources must satisfy

$$
\left[D^{\mu}, j_{\mu}\right]=i g t^{a}\left(\bar{\psi} t^{a} \eta-\bar{\eta} t^{a} \psi\right) .
$$

By differentiating the equations (5.30) and (5.27) with respect to the fields, one obtains relations between HTL refered as "QED-like" (or "tree-level like") Ward identities in Refs. [3, 2]. In particular, the transversality of the polarization tensor follows from eq. (5.30), after one differentiation with respect to $A_{\mu}$ :

$$
\delta \Pi_{\mu \nu} P^{\nu}=P^{\mu} \delta \Pi_{\mu \nu}=0 .
$$

Of course, this property can be easily verified on the explicit expression for $\delta \Pi_{\mu \nu}$ given in eq. (5.21). If we differentiate once again eq. (5.30), we derive the simple identity

$$
P_{1}^{\mu} \delta \Gamma_{\mu \nu \lambda}\left(P_{1}, P_{2}, P_{3}\right)=\delta \Pi_{\nu \lambda}\left(P_{3}\right)-\delta \Pi_{\nu \lambda}\left(P_{2}\right),
$$

which also follows from eqs. (5.22) and (5.21) above.

For amplitudes involving a quark pair, Ward identities result from differentiating eq. (5.27). For instance, by differentiating eq. (5.27) with respect to both $\psi$ and $\bar{\psi}$, and using eqs. (2.27) and (2.30), we recognize the QED-like Ward identity

$$
P^{\mu} \delta \Gamma_{\mu}\left(P ; K_{1}, K_{2}\right)=\delta \Sigma\left(K_{1}\right)-\delta \Sigma\left(K_{1}+P\right),
$$

which can also be deduced from the explicit expressions eqs. (5.14), (5.19) and (5.9). As a final example, consider the following identity, which results from eq. (5.27) after an extra differentiation with respect to $A_{\nu}^{b}$,

$$
\begin{aligned}
& P_{1}^{\mu} \delta \Gamma_{\mu \nu}^{a b}\left(P_{1}, P_{2} ; K_{1}, K_{2}\right)=i f^{a b c} \delta \Gamma_{\nu}^{c}\left(P_{1}+P_{2} ; K_{1}, K_{2}\right) \\
& \quad+\delta \Gamma_{\nu}^{b}\left(P_{2} ; K_{1}, K_{2}+P_{1}\right) t^{a}-t^{a} \delta \Gamma_{\nu}^{b}\left(P_{2} ; K_{1}+P_{1}, K_{2}\right),
\end{aligned}
$$

and can also be obtained directly from eqs. (5.14) and (5.16).

\subsection{Effective action for soft fields}

Using the results of Sec.5.1, one can rewrite the equations of motion for the average fields as

$$
i \not D \psi(X)-\omega_{0}^{2} \int \frac{d \Omega}{4 \pi} \psi \int d^{4} Y G_{r e t}(X, Y ; v) \psi(Y)=\eta(X),
$$


and

$$
\begin{array}{r}
{\left[D^{\nu}, F_{\nu \mu}(X)\right]^{a}-3 i \omega_{p}^{2} \int \frac{d \Omega}{4 \pi} v_{\mu} \int d^{4} Y \tilde{G}_{r e t}^{a b}(X, Y) \vec{v} \cdot \vec{E}^{b}(Y)} \\
=j_{\mu}^{a}(X)+g \bar{\psi}(X) \gamma_{\mu} t^{a} \psi(X)+j_{\mu}^{\psi a}(X)
\end{array}
$$

where $j_{\mu}^{\psi}(X)$ is the current induced by fermionic mean fields, given in eq. (5.17). In these equations, the fields are assumed to vanish adiabatically for $X_{0} \rightarrow-\infty$, which correspond to the plasma being initially in equilibrium. Of course, similar equations may be written to describe perturbations which vanish adiabatically in the remote future; then, the induced currents would be expressed in terms of the advanced Green's function.

In order to make contact with previous works, we shall now restrict the fields $\psi(X)$, $\bar{\psi}(X)$ and $A_{\mu}^{a}(X)$ to belong to a space $\mathcal{R}$ in which the operators $v \cdot D_{X}, v \cdot \tilde{D}_{X}$ and $\left(v \cdot \tilde{D}_{X}\right)^{2}$ never vanish. Specifying the boundary conditions becomes then unnecessary, and it is possible to deduce the equations of motion (5.36) and (5.37) by applying the minimum action principle to an effective action which can be written in a simple compact form. The space $\mathcal{R}$ is spaned by the fields which vanish exponentially for $\left|X_{0}\right| \rightarrow \infty$ and satisfy the conditions

$$
\begin{gathered}
\int_{-\infty}^{\infty} d u U(X, X-v u) \psi(X-v u)=0, \\
\int_{-\infty}^{\infty} d u \tilde{U}_{a b}(X, X-v u) F_{\mu \nu}^{b}(X-v u)=0, \\
\int_{-\infty}^{\infty} d u u \tilde{U}_{a b}(X, X-v u) F_{\mu \nu}^{b}(X-v u)=0 .
\end{gathered}
$$

These conditions guarantee that the fields have no components in the kernel of the covariant line derivatives. The left hand sides of eqs. (5.38)-(5.40) represent such components. For instance,

$$
\left(v \cdot D_{X}\right) \int_{-\infty}^{\infty} d u U(X, X-v u) \psi(X-v u)=0
$$

as one can easily verify (see e.g. eq. (4.16)). Physically, the conditions above imply that the fields do not suffer Landau damping. This is seen most simply in case of weak fields, where we can ignore the parallel transporter. Then the Fourier transforms of eq. (5.38) and (5.39) result in

$$
\psi\left(P^{0}=\vec{v} \cdot \vec{P}, \vec{P}\right)=0, \quad \text { and } \quad F_{\mu \nu}^{a}\left(P^{0}=\vec{v} \cdot \vec{P}, \vec{P}\right)=0
$$


Within $\mathcal{R}$, the inverse operators $1 / v \cdot D, 1 / v \cdot \tilde{D}$ and $1 /(v \cdot \tilde{D})^{2}$ are nonambiguous and represent indifferently the retarded or advanced Green's functions, which are equivalent on $\mathcal{R}$.

We write the effective action as $S_{\text {eff }}=S_{0}+S_{\text {ind }}$, where $S_{0}$ is the classical QCD action, (as given in eq. (2.4), but without the ghosts and the gauge fixing term), while $S_{\text {ind }}$ contains the effects of the interactions between the soft fields and the hard particles. In order that $S_{\text {eff }}$ generates the mean field equations of motion (5.36) and (5.37), one requires

$$
\delta S_{i n d} / \delta \bar{\psi}(X)=\eta^{i n d}(X), \quad \quad \delta S_{i n d} / \delta A_{a}^{\mu}(X)=j_{\mu}^{i n d a}(X)
$$

As proven in Appendix C, these conditions are satisfied by $S_{\text {ind }} \equiv S_{\mathrm{f}}+S_{\mathrm{b}}$, with

$$
S_{\mathrm{f}}=\omega_{0}^{2} \int \frac{d \Omega}{4 \pi} \int d^{4} X \int d^{4} Y \bar{\psi}(X)\left\langle X\left|\frac{\psi}{i(v \cdot D)}\right| Y\right\rangle \psi(Y),
$$

and

$$
S_{\mathrm{b}}=\frac{3}{2} \omega_{p}^{2} \int \frac{d \Omega}{4 \pi} \int d^{4} X \int d^{4} Y \operatorname{tr}\left[F_{\mu \lambda}(X)\left\langle X\left|\frac{v^{\mu} v_{\nu}}{(v \cdot \tilde{D})^{2}}\right| Y\right\rangle F^{\nu \lambda}(Y)\right],
$$

where the trace acts on color indices only. The action $S_{\mathrm{f}}$ accounts for all the polarization effects induced by soft fermionic mean fields, that is

$$
\delta S_{\mathrm{f}} / \delta \bar{\psi}(X)=\eta^{i n d}(X), \quad \delta S_{\mathrm{f}} / \delta A_{a}^{\mu}(X)=j_{\mu}^{\psi a}(X)
$$

while $S_{\mathrm{b}}$ satisfies

$$
\delta S_{\mathrm{b}} / \delta A_{a}^{\mu}(X)=j_{\mu}^{A a}(X)
$$

The effective action (5.44)-(5.45) coincides with the generating functional for HTL's first derived in [26, 27, 28] on the basis of gauge invariance. One sees from our analysis that $S_{\text {eff }}$ may be given the physical interpretation of the classical action which describes long wavelength excitations of the hot quark-gluon plasma, at leading order in the coupling $g$, but only as long as Landau damping is inoperative. One should emphasize that, indeed, the functional derivatives of $S_{\text {ind }}$ generate properly the induced currents only on $\mathcal{R}$ (see the discussion in Appendix 3). In cases where the boundary conditions matter, we have to recourse to the kinetic equations written in Sec.4.1 to solve for the induced sources appropriate to the given physical conditions (as done e.g. in Sec.4.2 for retarded conditions.) 


\section{Conclusions}

We have presented a consistent description of soft collective excitations of quark-gluon plasmas. This description relies on the hierarchy of Dyson-Schwinger equations, which in the leading order that we have considered, truncate at the level of 2-point functions. Our resulting equations take then the form of a coupled set of mean field and kinetic equations. The mean fields describe the soft excitations and have many features of classical degrees of freedom. The kinetic equations describe the dynamics of the hard plasma particles, which respond collectively to the action of the mean fields. This separation between mean fields and kinetic equations reflects the distinct roles played by the two types, hard and soft, degrees of freedom of the plasma, and also the fact that the dominant interactions at leading order are those between the hard particles and the mean fields.

As hard particles behave in a remarkably similar fashion, irrespective of whether they are quarks or gluons, they provide sources not only for average gauge fields, but also for average fermionic fields. And indeed the quark-gluon plasma sustains collective excitations with fermionic quantum numbers. That is, there exists excitations in which the soft fermion degrees of freedom oscillate over a long time scale. These oscillations are maintained by the hard particles coherently changing their quantum numbers from bosonic to fermionic and vice versa, and this over long space/time scales [34].

The excitations that we have studied are related to oscillations in the conserved currents associated with the QCD Lagrangian. There are thus oscillations in the baryon currents which result from the action of a fermionic mean field. There are also oscillations of color currents induced either by a fermionic field or by a gauge field. All these oscillations can be described in terms of fluctuations of density matrices which are generally non diagonal either in color space or in a space containing fermionic and bosonic degrees of freedom. The diagonal matrix elements, when they are non vanishing may be given the interpretation of classical distribution functions for particles of a given color.

The simple physical picture that we have just outlined is of course fully justified by the long technical developments presented in this paper, whose main features we now summarize.

The cornerstone of the whole approach is that collective modes develop at a particular energy scale, $g T$. The emergence of this scale is seen most clearly on the mean field equations which, in leading order, become universal equations, once length are measured in units of $1 / g T$ and energies in units of $g T$. It is the existence of this particular scale which allows us to organize our analysis of the Dyson-Schwinger equations, by controlling 
consistently at the same time the strength of the coupling, the wavelength and the amplitude of the collective oscillations, to eventually arrive at a consistent set of equations of motion which incorporate all leading order effects.

One outcome of our work is a set of consistent and gauge covariant kinetic equations for the plasma particles moving in background fields. As another by-product, we recover in a simple way all hard thermal loops which have been analyzed first in a diagrammatic approach. These are simply obtained from compact expressions for the induced sources, which may be regarded as generating functionals for the HTL's. Thus our approach provide a new and independent derivation of such generating functionals. It has also the advantage of clearly exhibiting the role of boundary conditions. In particular our induced currents are easily obtained with the retarded boundary conditions appropriate to the calculation of the causal response functions. The standard form of the HTL generating functional is recovered by restricting the fields to obey special conditions. The particular Ward identities which relate HTL's follow simply from conservation laws obeyed by the induced currents. Finally, the mean field equations provide expressions for the resummed soft propagators and vertices. 


\section{Appendix A}

We prove here that the propagation of soft mean fields with ghost quantum numbers does not give rise to polarization effects at leading order in $g$. That is, in this order, the induced sources can be ignored in the r.h.s. of eqs. (2.20) and (2.21) for the ghost mean fields. (In the diagrammatic language, this is equivalent to say that there are no "hard thermal loops" with ghost external lines [2].) It follows that the ghost fields vanish in the absence of external sources and they can be omitted in the equations of motion for the 2-point functions (see Section 3). For brevity, we present the explicit proof only in Feynman gauge and for vanishing gauge fields. The general case can be treated along the lines described at length in Appendix B.

The induced sources $C_{a}^{\text {ind }}$ and $\bar{C}_{a}^{\text {ind }}$ are related to the diagonal matrix elements of the ghost-gluon off-equilibrum propagators, $O_{\nu}^{a b}$ and $\bar{O}_{\nu}^{a b}$, by eqs. (2.44) and (2.45), which imply

$$
C^{i n d a}(X)=g f^{a b c}\left[\partial_{X}^{\nu} O_{\nu}^{b c}<(s, X)\right]_{s=0}=g f^{a b c} \int \frac{d^{4} k}{(2 \pi)^{4}} \partial_{X}^{\nu} \mathcal{O}_{\nu}^{b c}(k, X),
$$

and

$$
\bar{C}^{i n d a}(X)=g f^{a b c}\left[\partial_{s}^{\nu} \bar{O}_{\nu}^{b c<}(s, X)\right]_{s=0}=-i g f^{a b c} \int \frac{d^{4} k}{(2 \pi)^{4}} k^{\nu} \overline{\mathcal{O}}_{\nu}^{b c}(k, X) .
$$

Note that in going from eq. (2.45) to eq. (A.2) we have neglected the soft derivative $\partial_{X}$ in comparaison with $\partial_{s}$, whereas (A.1) is an exact rewriting of eq. (2.44).

The equations for $O_{\nu}^{a b}$ and $\bar{O}_{\nu}^{a b}$ are obtained by differentiating the mean fields equations with respect to the external sources, as explained in Sec.3.3. First, we differentiate the gauge field equation (2.18) with respect to $\bar{C}^{b}$ and $C^{b}$, and get, respectively,

$$
\mathcal{L}_{\mu \nu}^{a c}(y) O_{b c}^{\nu}(x, y)=-g f^{a c d}\left[\partial_{\mu}^{y} G^{b c}(x, y)\right]\left\langle\zeta^{d}(y)\right\rangle,
$$

and

$$
\mathcal{L}_{\mu \nu}^{a c}(x) \bar{O}_{c b}^{\nu}(x, y)=g f^{a c d} G^{c b}(x, y) \partial_{\mu}\left\langle\bar{\zeta}^{d}(x)\right\rangle .
$$

Then, we differentiate eqs. (2.20) and (2.21) for the ghost fields with respect $j_{b}^{\nu}$, and obtain

$$
\begin{aligned}
\partial_{x}^{2} O_{\nu}^{a b}(x, y)-g f^{a c d}\left\langle A_{\mu}^{c}(x)\right\rangle \partial_{x}^{\mu} O_{\nu}^{d b}(x, y) \\
\quad=g f^{a c d} \partial_{x}^{\mu}\left[D_{\mu \nu}^{c b}(x, y)\left\langle\zeta^{d}(x)\right\rangle\right],
\end{aligned}
$$


and

$$
\begin{aligned}
\partial_{y}^{2} \bar{O}_{\nu}^{b a}(x, y)-g f^{a c d} & \left\langle A_{\mu}^{c}(x)\right\rangle \partial_{y}^{\mu} \bar{O}_{\nu}^{b d}(x, y) \\
& =g f^{a c d} D_{\nu \mu}^{b c}(x, y) \partial^{\mu}\left\langle\bar{\zeta}^{d}(y)\right\rangle
\end{aligned}
$$

Let us consider first $C_{a}^{\text {ind }}$. This will be obtained after solving eqs. (A.3) and (A.5) for $O_{\nu}^{a b}$. As mentioned earlier, we choose $\left\langle A_{\mu}^{a}\right\rangle=0$, in order to simplify the discussion. Then, after doing approximations similar to those described at length in Section 3, we obtain for $O_{\nu}^{a b}$ the following two equations:

$$
\partial_{y}^{2} O_{\nu}^{a b<}(x, y)=g f^{a b c}\left(\partial_{\nu}^{y} \Delta(x-y)\right) \zeta^{c}(y)
$$

and

$$
\partial_{x}^{2} O_{\nu}^{a b<}(x, y)=-g f^{a b c} \partial_{\nu}^{x}\left[\Delta(x-y) \zeta^{c}(x)\right]
$$

The ghost mean fields are simply denoted here by $\zeta^{a}$ and $\bar{\zeta}^{a}$. These equations are further transformed into an equation for the Wigner function $\mathcal{O}_{\nu}^{a b}(k, X)$

$$
\left(k \cdot \partial_{X}\right) \mathcal{O}_{\nu}^{a b}(k, X)=i \frac{g}{2} f^{a b c} k_{\nu}\left(\partial_{X}^{\mu} \zeta^{c}\right)\left(\partial_{\mu}^{k} \Delta\right) .
$$

This equation includes consistently all the terms at leading order in $g$. It shows that $\mathcal{O}(k, X) \sim g(\zeta / T) \Delta$. One deduces then from eq. (A.1) that $C^{\text {ind }} \sim g^{3} T^{2} \zeta$, which is $g$ times smaller than the l.h.s. of eq. (2.20) for $\zeta, \partial^{2} \zeta \sim g^{2} T^{2} \zeta$. In fact, $C^{\text {ind }}$ is even more suppressed than this estimate suggests.

The corresponding proof for the anti-ghost mean field - i.e., for $\bar{C}^{\text {ind }}$ and $\bar{O}_{\nu}^{a b}$ - is slightly different, owing to the disymmetry with which the two kinds of ghost fields enter the QCD action (2.4). Now it is the abnormal propagator $\bar{O}_{\nu}^{a b}$ itself which vanishes at leading order. This can be easily verified by isolating the dominant terms in eqs. (A.4) and (A.6) for $\bar{O}_{\nu}^{a b}$.

\section{Appendix B}

In the derivation of the equations of motion in the main text, we have used systematically the Feynman gauge $(\lambda=1)$ in order to simplify the algebra. We prove now that this particular choice does not affect the final results: the equations that we have obtained for the mean fields and their induced sources preserve their form in any covariant 
gauge. As a consequence, the HTL's are independent of the gauge fixing parameter $\lambda$ for arbitrary (soft) external momenta.

The gauge fixing parameter $\lambda$ enters our calculations through the gluonic propagator, that is, either through the differential operator $\mathcal{L}_{\mu \nu}^{a b}(x)$, eq. (3.18), or through $D_{0 \mu \nu}^{<a b}(k) \equiv \delta^{a b} \rho_{\mu \nu}(k) N\left(k_{0}\right)$. Here $\rho_{\mu \nu}(k)$ is the spectral density in a general covariant gauge [32]

$$
\rho_{\mu \nu}(k)=\rho_{\mu \nu}^{F}(k)+\left(1-\lambda^{-1}\right) \rho_{\mu \nu}^{\lambda}(k),
$$

with $\rho_{\mu \nu}^{F}(k)=-g_{\mu \nu} \rho_{0}(k)$ the spectral density in Feynman gauge $\left(\rho_{0}(k) \equiv 2 \pi \epsilon\left(k_{0}\right) \delta\left(k^{2}\right)\right)$, while $\rho_{\mu \nu}^{\lambda}(k) \equiv-2 \pi \epsilon\left(k_{0}\right) k_{\mu} k_{\nu} \delta^{\prime}\left(k^{2}\right), \delta^{\prime}\left(k^{2}\right)$ denoting the derivative of $\delta\left(k^{2}\right)$ with respect to $k^{2}$. We also define $\Delta^{\prime}(k) \equiv 2 \pi \epsilon\left(k_{0}\right) \delta^{\prime}\left(k^{2}\right) N\left(k_{0}\right)$, by analogy with $\Delta(k)=\rho_{0}(k) N\left(k_{0}\right)$. By using $k^{2} \delta^{\prime}\left(k^{2}\right)=-\delta\left(k^{2}\right)$, we verify that

$$
k^{2} \Delta^{\prime}(k)=-\Delta(k)
$$

and consequently the gluon propagator can also be written as

$$
D_{0 \mu \nu}^{<}(k)=\left[g_{\mu \nu} k^{2}-\left(1-\lambda^{-1}\right) k_{\mu} k_{\nu}\right] \Delta^{\prime}(k) .
$$

We shall need later the following identity:

$$
k^{\mu} D_{0 \mu \nu}^{<}(k)=-\lambda^{-1} k_{\nu} \Delta(k)
$$

After these preparations, let us formulate the proposition to be proved below: in a general covariant gauge parametrized by $\lambda$, the $k$-space densities for the induced sources, i.e. $\not(k, X), \mathcal{J}_{\mathrm{f}}^{\psi}(k, X), \mathcal{J}_{\mathrm{b}}^{\psi}(k, X), \mathcal{J}_{\mathrm{f}}^{A}(k, X)$ and $\mathcal{J}_{\mathrm{b}}^{A}(k, X)$, are independent of $\lambda$ when consistently computed at leading order in $g$.

Note first that, since the gluon propagator is not involved in the equations for $\delta \mathcal{S}^{A}, \mathcal{J}_{\mathrm{f}}^{A}$ is trivially gauge independent. Furthermore, by examining the derivation of the equations satisfied by $\mathcal{J}_{\mathrm{f}}^{\psi}$ and $\mathcal{J}_{\mathrm{b}}^{\psi}$, one sees that these may depend on $\lambda$ only through $\mathbb{K}$ and $\mathcal{H}$. Thus, we only need to prove that $\mathbb{K}$ and $\mathcal{J}_{\mathrm{b}}^{A}$ are independent of $\lambda$.

We start with 仗. By following the same steps as in Sec.3.3, we derive from eq. (3.29) the equation which generalizes eq. (3.33) to arbitrary $\lambda$

$$
\left[k^{2}+i k \cdot\left(\partial_{X}+2 i g A\right)\right] \mathcal{K}_{\mu}^{a} \approx-g \Delta(k)\left[\not k \gamma_{\mu}-\left(1-\lambda^{-1}\right) k_{\mu}\right] t^{a} \psi
$$

where we have preserved only the terms of relevant order of magnitude. Similarly, starting from eq. (3.28) we get

$$
\left(k^{2}-i k \cdot \partial_{X}\right) \mathcal{K}_{\mu}^{a}-i g f^{a b c} \Gamma_{\mu \nu \rho \lambda} A_{c}^{\rho} k^{\lambda} \mathcal{K}_{b}^{\nu}+i(1-\lambda) k_{\mu} \tilde{\mathcal{K}}_{a} \approx g \tilde{\Delta}(k) \not k \gamma_{\mu} t^{a} \psi
$$


We have introduced here the function $\tilde{\mathcal{K}}_{a}(k, X)$, the Wigner transform of

$$
\tilde{K}_{a}(x, y) \equiv \partial_{y}^{\nu} K_{\nu a}^{<}(x, y)
$$

We now expand $\mathcal{K}=\mathcal{K}^{(0)}+\mathcal{K}^{(1)}+\ldots$, with $\mathcal{K}^{(1)} \sim g \mathcal{K}^{(0)}$, etc. At leading order, we recover from the previous equation the consistency conditions (3.35) and (3.39), that is, $k^{2} \mathcal{K}_{\mu}^{(0)}=0$ and $k^{\mu} \mathcal{K}_{\mu}^{(0)}=0$. At next to leading order, we subtract the two equations and obtain the generalization of eq. (3.37) to arbitrary $\lambda$

$$
\begin{gathered}
k \cdot\left(\partial_{X}+i g A\right) \mathcal{K}_{\mu}^{a}+\frac{g}{2} f^{a b c} \Gamma_{\mu \nu \rho \lambda} A_{c}^{\rho} k^{\lambda} \mathcal{K}_{b}^{\nu}-\frac{1-\lambda}{2} k_{\mu} \tilde{\mathcal{K}}_{a} \\
=i \frac{g}{2}[\Delta(k)+\tilde{\Delta}(k)] \not k \gamma_{\mu} t^{a} \psi-i \frac{g}{2}\left(1-\lambda^{-1}\right) \Delta(k) k_{\mu} t^{a} \psi,
\end{gathered}
$$

where $\mathcal{K} \equiv \mathcal{K}^{(0)}$. Because $k^{\mu} \mathcal{K}_{\mu}^{(0)}=0$, the leading order contribution to $\tilde{\mathcal{K}}_{a}$ (which enters eq. (B.8)) is $\sim g T \mathcal{K}^{(0)}$ :

$$
\tilde{\mathcal{K}}_{a}(k, X)=i k^{\nu} \mathcal{K}_{\nu}^{(1) a}(k, X)+\frac{1}{2} \partial_{X}^{\nu} \mathcal{K}_{\nu}^{(0) a}(k, X)
$$

Thus eq. (B.8) is consistent, i.e., all terms are of the same order of magnitude, $\sim g T^{2} \mathcal{K}^{(0)}$. The unknown function $\mathcal{K}^{(1)}$, as well as the $\lambda$ dependence disappear in the equation for $\mathbb{K}^{a} \equiv \gamma^{\mu} \mathcal{K}_{\mu a}^{(0)}$. These cancellations result from the identity

$$
\not k \tilde{\mathcal{K}}_{a}(k, X)=-i \frac{g}{\lambda} \not k \Delta(k) t^{a} \psi(X),
$$

which is proved by first differentiating eq. (3.29) with respect to $\partial_{y}^{\nu}$, and then using the identity $(\mathbb{B} .4)$, to get

$$
\not D_{x} \tilde{K}_{a}(x, y)=-i \frac{g}{\lambda} \gamma^{\mu} t^{a} \psi(x) \partial_{\mu} \Delta(x-y),
$$

which, in leading order in $g$, gives precisely eq. (B.10). Thus, we recover for $\mathbb{K}^{a}$ the Feynman gauge equation (3.40).

Consider now the gluonic current $\mathcal{J}_{\mathrm{b}}^{A}$. As discussed in Sec.3.7, $\mathcal{J}_{\mathrm{b}}^{A} \equiv \mathcal{J}_{\mathrm{g}}^{A}+\mathcal{J}_{G}+\mathcal{J}_{\Omega}$. Since, by construction, $\mathcal{J}_{G}$ is independent of $\lambda$, we need to prove that a similar property holds for the sum $\mathcal{J}_{\mathrm{g}}^{A}+\mathcal{J}_{\Omega}$.

From eq. (3.15)

$$
j_{\Omega \mu}^{a}(X)=g^{2} \hat{\Gamma}_{\mu \nu \rho \lambda}^{a b c d} A_{b}^{\nu}(X) D_{0 c d}^{<\rho \lambda}(0)=N g^{2}\left[g_{\mu \nu}\left(D_{0}^{<}\right)_{. \rho}^{\rho}(0)-\left(D_{0}^{<}\right)_{\mu \nu}(0)\right] A_{a}^{\nu}(X) .
$$

By using eq. (B.3), this can be written as a integral in $k$-space of $\mathcal{J}_{\Omega} \equiv \mathcal{J}_{\Omega}^{F}+\left(1-\lambda^{-1}\right) \mathcal{J}^{\lambda}$, where

$$
\mathcal{J}_{\Omega \mu}^{F a}=-g N(d-1) \Delta(k) A_{\mu}^{a}(X),
$$


is the Feynman gauge expression, eq. (3.17), while

$$
\mathcal{J}_{\mu}^{\lambda a}(k, X)=-g N\left(g_{\mu \nu} k^{2}-k_{\mu} k_{\nu}\right) \Delta^{\prime}(k) A_{a}^{\nu}(X)
$$

is a supplementary term for $\lambda \neq 1$.

As indicated in eq. (3.76), $\mathcal{J}_{\mathrm{g}}^{A}$ is determined by the quantities $\delta \mathcal{D}_{\mu \nu}^{a} k^{\nu}$ and $\delta \mathcal{D}_{\nu}^{a \nu}$. (To simplify the writing, we omit, here and below, the $A$-superscripts.) These quantities will be determined by following the same steps as in Sec.3.7. We start from eqs. (3.58) and (3.59), written for arbitrary $\lambda$ and $\psi=\bar{\psi}=0$. By using eq. (3.18) for $\mathcal{L}_{\mu \nu}^{a b}$, we write eq. (3.58) as

$$
\begin{aligned}
\left(\partial_{x}^{2} g_{\mu}^{\rho}-(1-\lambda) \partial_{\mu}^{x} \partial_{x}^{\rho}\right) D_{\rho \nu}^{a b<}<(x, y) & =-g f^{a d c}\left[\Gamma_{\mu \sigma \rho \lambda} A_{c}^{\rho}(x) \partial_{x}^{\lambda}-\Gamma_{\mu \rho \lambda \sigma}\left(\partial_{x}^{\lambda} A_{c}^{\rho}(x)\right)\right] D_{d \nu}^{\sigma b<}(x, y) \\
& +g^{2} \hat{\Gamma}_{\mu \sigma \rho \lambda}^{a d c e} A_{c}^{\rho}(x) A_{e}^{\lambda}(x) D_{d \nu}^{\sigma b<}(x, y) .
\end{aligned}
$$

We shall refer to this equation as (I). We have a similar equation deduced from (3.59) and to which we refer below as (II). It is obtained from (I) by exchanging, respectively, $x$ and $y, a$ and $b$, and $\mu$ and $\nu$. We separate the induced part of $D$ by setting $D^{<} \equiv D_{0}^{<}+\delta D$, with $\delta D \sim g D_{0}$ for $A \sim T$. Note that $D_{0}^{<}$does not contribute to the l.h.s. of (I) and (II), because it satisfies $D_{0}^{-1} D_{0}^{<}=0 ;\left(D_{0}^{-1}\right.$ is the free inverse propagator, eq. (2.28) ). We expand $\delta D=\delta D^{(0)}+\delta D^{(1)}+\ldots$, where $\delta D^{(1)} \sim g \delta D^{(0)}$, etc... At leading order, (I) and (II) lead to the consistency condition which generalizes eq. (3.61) for $\lambda \neq 1$

$$
k^{2} \delta \mathcal{D}_{\mu \nu}^{a}-(1-\lambda) k_{\mu} k^{\rho} \delta \mathcal{D}_{\rho \nu}^{a}=g \Gamma_{\mu \sigma \rho \lambda} A_{c}^{\rho}(X) k^{\lambda} D_{0 \nu}^{<\sigma}(k),
$$

where we have set (see eq. (3.74))

$$
\delta \mathcal{D}_{\mu \nu}^{a}(k, X) \equiv(i / N) f^{a b c} \delta \mathcal{D}_{\mu \nu}^{(0) b c}(k, X) .
$$

At next-to-leading order, we encounter terms involving the unknown function $\delta D^{(1)}$. For instance, $\left(k \cdot \partial_{X}\right) \delta D_{\mu \nu}^{(0)} \sim k^{2} \delta D_{\mu \nu}^{(1)} \sim k_{\mu} k^{\rho} \delta D_{\rho \nu}^{(1)}$ in the l.h.s. of eq. (I). Contrary to what happened in the Feynman gauge, now such terms do not disappear totally in the difference of eqs. (I) and (II). Nor do the terms proportional to $\hat{\Gamma}$ in the r.h.s.'s of Eqs.(I) and (II). However, things simplify in the equations for the functions of interest, $\delta \mathcal{D}_{\mu \nu}^{a} k^{\nu}$ and $\delta \mathcal{D}_{\nu}^{a \nu}$.

Consider first the equations for $\delta \mathcal{D}_{\nu}^{a \nu}$. When traced over space-time indices, the terms depending on $\delta D^{(1)}$, as well as those coming from the four gluons interactions, become identical in eqs. (I) and (II) (at the order of interest). Then, by taking the difference of these two equations, we get

$$
\begin{aligned}
& {\left[k \cdot D_{X}, \delta \mathcal{D}_{\nu}^{\nu}\right]^{a}+g f^{a b c}\left(A_{b}^{\mu} \delta \mathcal{D}_{\mu \nu}^{c} k^{\nu}\right)-(1-\lambda)\left(\partial_{X}^{\mu} \delta \mathcal{D}_{\mu \nu}^{a} k^{\nu}\right)} \\
& \quad=g(d-1) k^{\nu}\left(\partial_{\mu} A_{\nu}^{a}\right)\left(\partial^{\mu} \Delta\right)+g\left(1-\lambda^{-1}\right)\left(\partial_{\mu} A_{\nu}^{a}\right)\left(k^{\mu} k^{\nu}-g^{\mu \nu} k^{2}\right) \Delta^{\prime}(k),
\end{aligned}
$$


which generalizes eq. (3.77) for arbitrary $\lambda$.

Similarly, we shall prove shortly that $\delta \mathcal{D}_{\mu \nu}^{a} k^{\nu}$ satisfies

$$
\begin{array}{r}
\left(k \cdot \partial_{X}\right)\left(\delta \mathcal{D}_{\mu \nu}^{a} k^{\nu}\right)-\frac{3}{4} g f^{a b c}\left(k \cdot A^{b}\right)\left(\delta \mathcal{D}_{\mu \nu}^{c} k^{\nu}\right)+\frac{g}{4} f^{a b c} k_{\mu}\left(A^{b} \cdot \delta \mathcal{D}^{c} \cdot k\right) \\
=\frac{g}{2 \lambda}\left(k_{\mu} k_{\nu}-g_{\mu \nu} k^{2}\right)\left(\partial^{\rho} A_{a}^{\nu}\right)\left(\partial_{\rho} \Delta\right)-\frac{3}{4 \lambda} g^{2} f^{a b c}\left(k \cdot A^{b}\right) A_{\mu}^{c} \Delta .
\end{array}
$$

Thus, $\lambda \delta \mathcal{D}_{\mu \nu}^{a} k^{\nu}$ satisfies the same equation as $\delta \mathcal{D}_{\mu \nu}^{a} k^{\nu}$ does in Feynman gauge (see eq. (3.80)), and, according to eq. (3.82),

$$
\lambda \delta \mathcal{D}_{\mu \nu}^{A a} k^{\nu}(k, X)=g A_{\mu}^{a}(X) \Delta(k)-k_{\mu} \delta \mathcal{G}^{a}(k, X) .
$$

We can use this relation to eliminate the terms dependent on $\delta \mathcal{D}_{\mu \nu}^{a} k^{\nu}$ in eq. (B.18), which becomes then

$$
\begin{aligned}
{\left[k \cdot D_{X},\right.} & \left.\delta \mathcal{D}_{\nu}^{\nu}-\left(1-\lambda^{-1}\right)\left(\delta \mathcal{G}+g(k \cdot A) \Delta^{\prime}(k)\right)\right]^{a} \\
& =g(d-1) k_{\nu}\left(\partial^{\rho} A_{a}^{\nu}\right)\left(\partial_{\rho}^{k} \Delta\right)+g f^{a b c}\left(k \cdot A^{b}\right) \delta \mathcal{G}^{c} .
\end{aligned}
$$

This equation shows that $\delta \mathcal{D}_{\nu}^{\nu}-\left(1-\lambda^{-1}\right)\left(\delta \mathcal{G}+g(k \cdot A) \Delta^{\prime}(k)\right)$ is independent of $\lambda$; for $\lambda=1$, (B.21) reduces to eq. (3.84).

By using the expression $(\overline{B .20})$ in eq. $(3.76)$, we obtain the following expression for $\mathcal{J}_{\mathrm{g}} \equiv \mathcal{J}_{\mathrm{g}}^{A}$ :

$$
\mathcal{J}_{\mathrm{g} \mu}^{a}(k, X)=\frac{g}{\lambda} N \Delta(k) A_{\mu}^{a}(X)-N k_{\mu}\left(\delta \mathcal{D}_{\nu}^{a \nu}(k, X)+\frac{1}{\lambda} \delta \mathcal{G}^{a}(k, X)\right) .
$$

It is now straightforward to verify that $\mathcal{J}_{\mathrm{g}}=\mathcal{J}_{\mathrm{g}}^{F}-\left(1-\lambda^{-1}\right) \mathcal{J}^{\lambda}$, where $\mathcal{J}_{\mathrm{g}}^{F}$ is the expression obtained for $\mathcal{J}_{\mathrm{g}}^{A}$ in Feynman gauge, eq. (3.83), while $\mathcal{J}^{\lambda}$ is given by eq. (B.14). Indeed, from eqs. (B.14) and (B.22), we see that

$$
\begin{aligned}
& \mathcal{J}_{\mathrm{g} \mu}^{a}+\left(1-\lambda^{-1}\right) \mathcal{J}_{\mu}^{\lambda a}=g N \Delta(k) A_{\mu}^{a} \\
& \quad-N k_{\mu}\left\{\delta \mathcal{D}_{\nu}^{a \nu}-\left(1-\lambda^{-1}\right)\left(\delta \mathcal{G}^{a}+g\left(k \cdot A^{a}\right) \Delta^{\prime}(k)\right)+\delta \mathcal{G}^{a}\right\},
\end{aligned}
$$

where the r.h.s. is, according to eq. (B.21), independent of $\lambda$, and equal to $\mathcal{J}_{\mathrm{g}}^{F}$ (compare, in this respect, eqs. (B.21) and (3.84) $)$. Thus, $\mathcal{J}_{\mathrm{g}}+\mathcal{J}_{\Omega}=\mathcal{J}_{\mathrm{g}}^{F}+\mathcal{J}_{\Omega}^{F}$, as claimed.

To complete our proof, we still have to establish eq. (B.19) for $\delta \mathcal{D}_{\mu \nu}^{a} k^{\nu}$. The technical difficulty is the elimination of the terms involving the next-to-leading order correction, $\delta D^{(1)} \sim g \delta D^{(0)}$. In order to do this, we consider the equation satisfied by the derivatives of $\delta D(x, y)$. First, we obtain an equation for $\partial_{y}^{\nu} \delta D_{\mu \nu}^{a b}$ by differentiating Eqs.(I) and (II) with respect to $\partial_{y}^{\nu}$ and subtracting them. The l.h.s. of the resulting equation is

$$
\left(\partial_{x}^{2}-\partial_{y}^{2}\right)\left(\partial_{y}^{\nu} \delta D_{\mu \nu}^{a b}\right)-(1-\lambda) \partial_{\mu}^{x}\left(\partial_{x}^{\rho} \partial_{y}^{\lambda} \delta D_{\rho \lambda}^{a b}\right)
$$


At leading order, $\delta D^{(1)}$ appears only in the second term: $\partial_{\mu}^{s}\left(\partial_{s}^{\rho} \partial_{s}^{\lambda} \delta D_{\rho \lambda}^{(1)}\right) \sim g^{2} T^{3} D_{0}$, i.e., of the order of $\left(\partial_{x}^{2}-\partial_{y}^{2}\right)\left(\partial_{y}^{\nu} \delta D_{\mu \nu}^{a b}\right) \approx-2\left(\partial_{s} \cdot \partial_{X}\right)\left(\partial_{s}^{\nu} \delta D_{\mu \nu}^{(0) a b}\right) \sim g^{2} T^{3} D_{0}$ (recall that $\left.\left(\partial_{s}^{\rho} \partial_{s}^{\lambda} \delta D_{\rho \lambda}^{(0)}\right)=0\right)$. By the same procedure, we get an equation $\partial_{x}^{\nu} \delta D_{\nu \mu}^{a b}$, whose l.h.s. is

$$
\left(\partial_{x}^{2}-\partial_{y}^{2}\right)\left(\partial_{x}^{\nu} \delta D_{\nu \mu}^{a b}\right)+(1-\lambda) \partial_{\mu}^{y}\left(\partial_{x}^{\rho} \partial_{y}^{\lambda} \delta D_{\rho \lambda}^{a b}\right) .
$$

Then, eq. (B.19) for $\delta \mathcal{D}_{\mu \nu}^{a} k^{\nu}$ is obtained by subtracting the equations corresponding to (B.25) and (B.24). The l.h.s. is, at the relevant order,

$$
4\left(\partial_{s} \cdot \partial_{X}\right)\left(\partial_{s}^{\nu} \delta D_{\mu \nu}^{(0) a b}\right)+(1-\lambda) \partial_{\mu}^{X}\left(\partial_{x}^{\rho} \partial_{y}^{\lambda} \delta D_{\rho \lambda}^{a b}\right) .
$$

The second term in eq. (B.26) is now negligible at this order, as it is $\sim g^{3} T^{3} D_{0}$. Getting the r.h.s. of this equation presents no difficulty. A Fourier transform with respect to $s$ then yields eq. (B.19) for $\delta \mathcal{D}_{\mu \nu}^{a} k^{\nu}$.

\section{Appendix C}

We prove here that the induced sources $\eta^{\text {ind }}$, eq. (4.17), and $j_{\mu}^{\text {ind }}=j_{\mu}^{\psi}+j_{\mu}^{A}$, eqs. (4.27) and (4.39), are generated formally by $S_{\text {ind }}=S_{\mathrm{f}}+S_{\mathrm{b}}$, with $S_{\mathrm{f}}$ and $S_{\mathrm{b}}$ given, respectively, by eqs. (5.44) and (5.45), if the average fields $\psi(X)$ and $A_{\mu}^{a}(X)$ belong to the space $\mathcal{R}$, defined by eqs. (5.38)-(5.40).

Recall first that, within $\mathcal{R}$, the inverse operators $1 / v \cdot D$ and $1 /(v \cdot \tilde{D})^{2}$ which appear in eqs. (5.44) and (5.45) are unambiguous and represent indifferently the retarded or advanced Green's functions, which are equivalent on $\mathcal{R}$. For instance, we can easily verify, using eqs. (5.4) and (5.5), that

$$
\int d^{4} Y\left(G_{r e t}(X, Y ; v)-G_{a d v}(X, Y ; v)\right) \psi(Y)=-i \int_{-\infty}^{\infty} d u U(X, X-v u) \psi(X-v u)=0,
$$

where the last equality follows from eq. (5.38). This allows us, when doing formal operations in $\mathcal{R}$, to replace $1 / v \cdot D$ by the retarded Green's function, which has a simple expression in terms of the parallel transporter:

$$
\int d^{4} Y\left\langle X\left|\frac{1}{v \cdot D}\right| Y\right\rangle \psi(Y)=\int_{0}^{\infty} d u U(X, X-v u) \psi(X-v u) .
$$

This is convenient as it allows us to use nice identities satisfied by the parallel transporters. Similarly, we can identify on $\mathcal{R}$ the operators $1 /(v \cdot \tilde{D})^{2}$ and

$$
\begin{aligned}
\tilde{G}_{r e t}^{(2)}(X, Y ; v) & \equiv-\int d^{4} Z \tilde{G}_{r e t}(X, Z ; v) \tilde{G}_{r e t}(Z, Y ; v) \\
& =\theta\left(X^{0}-Y^{0}\right)\left(X^{0}-Y^{0}\right) \delta^{(3)}\left(\vec{X}-\vec{Y}-\vec{v}\left(X^{0}-Y^{0}\right)\right) \tilde{U}(X, Y) .
\end{aligned}
$$


Thus, on $\mathcal{R}$,

$$
\int d^{4} Y\left\langle X\left|\frac{1}{(v \cdot \tilde{D})^{2}}\right| Y\right\rangle F_{\mu \nu}(Y)=\int_{0}^{\infty} d u u U(X, X-v u) F_{\mu \nu}(X-v u) U(X-v u, X) .(\mathrm{C} .4)
$$

We conclude that the previous expressions for $S_{\mathrm{f}}$, eq. (5.44), and for $S_{\mathrm{b}}$, eq. (5.45), are equivalent respectively to

$$
S_{\mathrm{f}}=-i \omega_{0}^{2} \int \frac{d \Omega}{4 \pi} \int d^{4} X \int_{0}^{\infty} d u \bar{\psi}(X) \psi U(X, X-v u) \psi(X-v u),
$$

and

$$
\begin{aligned}
S_{\mathrm{b}}=\frac{3}{2} \omega_{p}^{2} \int \frac{d \Omega}{4 \pi} \int d^{4} X \int_{0}^{\infty} d u u \\
\operatorname{tr}\left\{v^{\mu} F_{\mu \lambda}(X) U(X, X-v u) v_{\nu} F^{\nu \lambda}(X-v u) U(X-v u, X)\right\},
\end{aligned}
$$

where the trace acts on color indices only.

Let us prove now that, when the conditions (5.38)-(5.40) hold, the induced sources are indeed generated from the effective action, according to eqs. (5.46)-(5.47). The first equality in eq. (5.46) follows immediatly from eqs. (C.5) for $S_{\mathrm{f}}$ and (4.17) for $\eta^{\text {ind }}$. To prove the second identity, which relates $S_{\mathrm{f}}$ to $j_{\mu}^{\psi}$, we differentiate eq. (5.44) with respect to $A_{a}^{\mu}(X)$, using

$$
\frac{\delta}{\delta A_{a}^{\mu}(X)}\left\langle Y_{1}\left|\frac{1}{i(v \cdot D)}\right| Y_{2}\right\rangle=g v_{\mu}\left\langle Y_{1}\left|\frac{1}{i(v \cdot D)}\right| X\right\rangle t^{a}\left\langle X\left|\frac{1}{i(v \cdot D)}\right| Y_{2}\right\rangle
$$

and obtain

$$
\begin{aligned}
\frac{\delta S_{\mathrm{f}}}{\delta A_{a}^{\mu}(X)}=g \omega_{0}^{2} & \int \frac{d \Omega}{4 \pi} v_{\mu} \int d^{4} Y_{1} \int d^{4} Y_{2} \\
& \left\{\bar{\psi}\left(Y_{1}\right)\left\langle Y_{1}\left|\frac{1}{i(v \cdot D)}\right| X\right\rangle \psi t^{a}\left\langle X\left|\frac{1}{i(v \cdot D)}\right| Y_{2}\right\rangle \psi\left(Y_{2}\right)\right\}
\end{aligned}
$$

This is precisely the expression (5.17) for $j_{\mu}^{\psi}$ (recall that, on $\mathcal{R}, G_{a d v}(X, Y ; v)=G_{r e t}(X, Y ; v)$ $=\langle X|1 / i(v \cdot D)| Y\rangle)$.

We now turn to the verification of eq. (5.47), which proceeds in three steps. In order to do this, it is convenient to use the expression of $S_{\mathrm{b}}$ in terms of the parallel transporter.

i) We differentiate functionally $S_{\mathrm{b}}$, eq. (C.6), with respect to $A_{\mu}^{a}(X)$ and write the result of this operation as

$$
\delta S_{\mathrm{b}} \equiv \delta S_{\mathrm{b}}^{(1)}+\delta S_{\mathrm{b}}^{(2)}
$$


The first piece comes from differentiating the $F_{\mu \nu}$ 's in eq. (C.6),

$$
\begin{aligned}
& \delta S_{\mathrm{b}}^{(1)}=\frac{3}{4} \omega_{p}^{2} \int \frac{d \Omega}{4 \pi} \int d^{4} X \int_{0}^{\infty} d u u v^{\mu} \delta F_{\mu \lambda}^{a}(X) \\
& \quad\left\{\tilde{U}_{a b}(X, X-v u) v_{\nu} F_{b}^{\nu \lambda}(X-v u)+\tilde{U}_{a b}(X, X+v u) v_{\nu} F_{b}^{\nu \lambda}(X+v u)\right\} .
\end{aligned}
$$

The second term, $\delta S_{\mathrm{b}}^{(2)}$, comes from the parallel transporter,

$$
\delta S_{\mathrm{b}}^{(2)}=\frac{3}{4} \omega_{p}^{2} \int \frac{d \Omega}{4 \pi} \int d^{4} X \int_{0}^{\infty} d u u v^{\mu} F_{\mu \lambda}^{a}(X)\left[\delta \tilde{U}_{a b}(X, X-v u)\right] v_{\nu} F_{b}^{\nu \lambda}(X-v u) .
$$

From eq. (4.14), we derive

$$
\begin{aligned}
\delta \tilde{U}_{a b}(X, X-v u) & =\int d^{4} Y \frac{\delta \tilde{U}_{a b}(X, X-v u)}{\delta A_{\rho}^{a}(Y)} \delta A_{\rho}^{a}(Y) \\
& =-i g u \int_{0}^{1} d s[\tilde{U}(X, Z) v \cdot \delta \tilde{A}(Z) \tilde{U}(Z, X-v u)]_{a b}
\end{aligned}
$$

with $\tilde{A}_{\mu} \equiv T^{a} A_{\mu}^{a}$ and $Z^{\mu}(s)=X^{\mu}-u(1-s) v^{\mu}$. We introduce (C.12) into (C.11) and derive, after elementary changes of integration variables,

$$
\begin{aligned}
& \delta S_{\mathrm{b}}^{(2)}=-i g \frac{3}{4} \omega_{p}^{2} \int \frac{d \Omega}{4 \pi} \int d^{4} X \int_{0}^{\infty} d u \int_{0}^{\infty} d t(u+t) \\
& \left\{v^{\mu} F_{\mu \lambda}^{a}(X+v t)[\tilde{U}(X+v t, X) v \cdot \delta \tilde{A}(X) \tilde{U}(X, X-v u)]_{a b} v_{\nu} F_{b}^{\nu \lambda}(X-v u)\right\}(C .1
\end{aligned}
$$

ii) We now derive a new expression for the induced current $j_{\mu}^{A}$, which will make easier the comparaison with $\delta S_{\mathrm{b}} / \delta A^{\mu}$. We note that eq. (4.9) can be solved in the form

$$
\mathcal{J}_{\mu}^{A a}(k, X)=g v_{\mu}^{\prime} k_{\nu} \partial_{\rho} \Delta_{e f f}(k) \int_{0}^{\infty} d u \tilde{U}_{a b}\left(X, X-v^{\prime} u\right) F_{b}^{\nu \rho}\left(X-v^{\prime} u\right)
$$

whith $v_{\mu}^{\prime} \equiv k_{\mu} / k_{0}$ and $\Delta_{e f f}(k) \equiv 2 N_{f} \tilde{\Delta}(k)+(d-2) N \Delta(k)$. The expression (4.39) of the induced current $j_{\mu}^{A}$ is obtained from (C.14) after a direct integration over $k$. Alternatively, one can integrate by parts with respect to $k$ and derive, after some elementary calculations,

$$
j_{\mu}^{A a}(X)=-\frac{3}{2} \omega_{p}^{2} \int \frac{d \Omega}{4 \pi} v_{\nu} \int_{0}^{\infty} d u \frac{\partial}{\partial v^{\rho}}\left[v_{\mu} \tilde{U}_{a b}(X, X-v u) F_{b}^{\nu \rho}(X-v u)\right] .
$$

Here, $v_{\mu} \equiv\left(1, \vec{k} / \epsilon_{k}\right)$, but the four components have to be considered as independent variables before taking the derivative $\partial / \partial v^{\rho}$. The integrand in eq. (C.15) is further transformed by writing

$$
\begin{aligned}
\frac{\partial}{\partial v^{\rho}}\left[\tilde{U}_{a b}(X, X-v u) F_{b}^{\nu \rho}(X-v u)\right] & =-u \frac{\partial}{\partial X^{\rho}}\left[\tilde{U}_{a b}(X, X-v u) F_{b}^{\nu \rho}(X-v u)\right] \\
& +u\left[\frac{\partial}{\partial X^{\rho}} \tilde{U}_{a b}(X, Y)\right]_{Y=X-v u} F_{b}^{\nu \rho}(X-v u),
\end{aligned}
$$


and the second term in the r.h.s. is evaluated by using [22]

$$
\left.\tilde{D}_{X}^{\rho} \tilde{U}(X, Y)\right|_{Y=X-v u}=i g u \int_{0}^{1} d s s\left[\tilde{U}(X, Z) v_{\lambda} \tilde{F}^{\lambda \rho}(Z) \tilde{U}(Z, X-v u)\right]
$$

with $\tilde{F}^{\lambda \rho} \equiv T^{a} F_{a}^{\lambda \rho}$ and $Z^{\mu}(s)=X^{\mu}-u(1-s) v^{\mu}$. Introducing (C.16) and (C.17) into (C.15) and using the identity

$$
\int_{0}^{\infty} d u\left[1-u\left(v \cdot \tilde{D}_{X}\right)\right]_{a b} \tilde{U}_{b c}(X, X-v u) F_{c}^{\nu \rho}(X-v u)=0,
$$

we derive the following expression for the induced current:

$$
j_{\mu}^{A}(X)=j_{\mu}^{(1)}(X)+j_{\mu}^{(2)}(X)
$$

with

$$
\begin{aligned}
& j_{\mu}^{(1) a}(X)=-\frac{3}{2} \omega_{p}^{2} \int \frac{d \Omega}{4 \pi} \int_{0}^{\infty} d u u \\
& \quad\left[g_{\mu \rho}\left(v \cdot \tilde{D}_{X}\right)-v_{\mu} \tilde{D}_{\rho}\right]_{a b} \tilde{U}_{b c}(X, X-v u) v_{\nu} F_{c}^{\nu \rho}(X-v u),
\end{aligned}
$$

and

$$
\begin{aligned}
& j_{\mu}^{(2) a}(X)=-i g \frac{3}{2} \omega_{p}^{2} \int \frac{d \Omega}{4 \pi} v_{\mu} \int_{0}^{\infty} d u u^{2} \int_{0}^{1} d s s \\
& {\left[\tilde{U}(X, Z) v^{\lambda} \tilde{F}_{\lambda \rho}(Z) \tilde{U}(Z, X-v u)\right]_{a b} v_{\nu} F_{b}^{\nu \rho}(X-v u) .}
\end{aligned}
$$

After using the identity

$$
\tilde{U}(X, Z) T^{a} \tilde{U}(Z, X)=\tilde{U}_{a b}(Z, X) T^{b}
$$

we make a change of integration variables in eq. (C.21) and finally obtain

$$
\begin{aligned}
& j_{\mu}^{(2) a}(X)=i g \frac{3}{4} \omega_{p}^{2} \int \frac{d \Omega}{4 \pi} v_{\mu} \int_{0}^{\infty} d u \int_{0}^{\infty} d t(u-t) \\
& \quad\left\{v^{\rho} F_{\rho \lambda}^{b}(X-v t)\left[\tilde{U}(X-v t, X) T^{a} \tilde{U}(X, X-v u)\right]_{b c} v_{\nu} F_{c}^{\nu \lambda}(X-v u)\right\} .
\end{aligned}
$$

iii) It is now straightforward to verify that, for gauge fields $A_{\mu}^{a}$ in $\mathcal{R}$, the following equalities are true

$$
\delta S_{\mathrm{b}}^{(1)} / \delta A_{a}^{\mu}(X)=j_{\mu}^{(1) a}(X), \quad \delta S_{\mathrm{b}}^{(2)} / \delta A_{a}^{\mu}(X)=j_{\mu}^{(2) a}(X)
$$

The first equality results from eqs. (C.10) and (C.20), by recalling that

$$
\delta F_{\mu \nu}(X)=\tilde{D}_{\mu} \delta A_{\nu}(X)-\tilde{D}_{\nu} \delta A_{\mu}(X)
$$


and by using the identity

$$
\int_{0}^{\infty} d u u \tilde{U}_{a b}(X, X-v u) F_{\mu \nu}^{b}(X-v u)=\int_{0}^{\infty} d u u \tilde{U}_{a b}(X, X+v u) F_{\mu \nu}^{b}(X+v u),(\mathrm{C} .26)
$$

which follows from the condition (5.40). As for the second equality (C.24), we note that (C.13) and (C.23) imply

$$
\begin{aligned}
& \delta S_{\mathrm{b}}^{(2)} / \delta A_{a}^{\mu}(X)-j_{\mu}^{(2) a}(X)=-i g \frac{3}{4} \omega_{p}^{2} \int \frac{d \Omega}{4 \pi} v_{\mu} \int_{-\infty}^{\infty} d t \int_{0}^{\infty} d u(u+t) \\
& \quad\left\{v^{\rho} F_{\rho \lambda}^{b}(X+v t)\left[\tilde{U}(X+v t, X) T^{a} \tilde{U}(X, X-v u)\right]_{b c} v_{\nu} F_{c}^{\nu \lambda}(X-v u)\right\} .
\end{aligned}
$$

Again, this vanishes because of the conditions (5.39) and (5.40). This completes our proof.

\section{References}

[1] R. D. Pisarski, Phys. Rev. Lett.63 (1989) 1129.

[2] E. Braaten and R. D. Pisarski, Nucl. Phys. B337 (1990) 569; Nucl. Phys. B339 (1990) 310.

[3] J. Frenkel and J.C. Taylor, Nucl. Phys. B334 (1990) 199.

[4] G. Baym, H. Monien, C.J. Pethick, and D.G. Ravenhall, Phys. Rev. Lett.64 (1990) 1867.

[5] E. Braaten and R. D. Pisarski, Phys. Rev. Lett.64 (1990) 1338.

[6] R. Kobes, G. Kunstater and A. Rebhan, Nucl. Phys. B355 (1991) 1.

[7] E. Braaten and R. D. Pisarski, Phys. Rev. D42 (1990) 2156.

[8] E. Braaten and R. D. Pisarski, Phys. Rev. D46 (1992) 1829; R. D. Pisarski, Brookhaven preprint (December, 1992).

[9] H. Heiselberg, G. Baym, C.J. Pethick and J. Popp, Nucl. Phys. A544 (1992) 569c.

H. Heiselberg and C.J. Pethick, Phys. Rev. D47 (1993) R769.

[10] E. Braaten and M.H. Thoma, Phys. Rev. D44 (1991) 1298.

[11] E. Braaten, R.D. Pisarski, and T.C.Yuan, Phys. Rev. Lett.64 (1990) 2242; H.A. Weldon, Phys. Rev. D42 (1990) 2384; Phys. Rev. Lett.66 (1991) 293; S.M.H. Wong, Cambridge University preprint DAMTP 92-54. 
[12] R.R. Parwani, Phys. Rev. D45 (1992) 4695.

[13] E.V.Shuryak, $\quad$ Zh.E.T.F.74 (1978)408; $\quad$ Sov. Phys. JETP47 (1978)212; Phys.Rep.61(1980) 71.

[14] O.K. Kalashnikov and V.V. Klimov, Sov. J. Nucl. Phys.31 (1980) 699; V.V. Klimov, Sov. Phys. JETP55 (1982) 199; Sov. J. Nucl. Phys.33 (1981) 934.

[15] H.A. Weldon, Phys. Rev. D26 (1982) 1394; Phys. Rev. D26 (1982) 2789.

[16] J.P. Blaizot and E. Iancu, Nucl. Phys. B390 (1993) 589.

[17] J.P. Blaizot and E. Iancu, Phys. Rev. Lett.70 (1993) 3376.

[18] E.M. Lifshitz and L.P. Pitaevskii, Physical Kinetics, (Pergamon Press, Oxford, 1981).

[19] V.P. Silin, Sov. Phys. JETP11 (1960) 1136.

[20] M. Gell-Mann and K.A. Brueckner, Phys.Rev.106 (1957) 364.

[21] U. Heinz, Phys. Rev. Lett.51 (1983) 351; J. Winter, J.Phys. (Paris) Suppl.45 (1984) C4-53.

[22] H.-Th. Elze, M.Gyulassy and D. Vasak, Nucl. Phys. B276 (1986) 706; Phys. Lett. B177 (1986) 402; H.-Th. Elze, Z.Phys.C 38 (1988) 211.

[23] H.-Th. Elze and U. Heinz, Phys. Repts.183 (1989) 81.

[24] S. Mrówczyński, Phys. Rev. D39 (1989) 1940.

[25] P. Bożek and M. Płoszajczak, Phys. Rev. D41 (1990) 634.

[26] J.C.Taylor and S.M.H.Wong, Nucl. Phys. B346 (1990) 115.

[27] E. Braaten and R.D. Pisarski, Phys. Rev. D45 (1992) R1827.

[28] J. Frenkel and J.C. Taylor, Nucl. Phys. B374 (1992) 156.

[29] R. Efraty and V.P. Nair, Phys. Rev. Lett.68 (1992) 2891.

[30] H.A. Weldon, "Hard thermal Loops and their Noether Currents", in Proceedings of the Workshop on Finite Temperature Field Theory, Winipeg Institute for Theoretical Physics, July 92, to appear in Canadian Journal of Physics. 
[31] L.Kadanoff and G.Baym, Quantum Statistical Mechanics, (Benjamin, New York,1962).

[32] N. Landsman and Ch. Van Weert, Phys. Repts.145 (1987) 142.

[33] G.Baym, J.P.Blaizot and B.Svetitsky, Phys. Rev. D46 (1992) 4043.

[34] J.P.Blaizot and J.-Y. Ollitrault, "Collective Fermionic Excitations in Systems with Large Chemical Potential", Saclay preprint S.Ph-T/93-018, to appear in Phys. Rev. D. 


\section{FIGURE CAPTIONS}

Fig.1:

Diagrammatic illlustration of the equation of motion (2.18) for the average gauge field $\left\langle A_{\mu}(x)\right\rangle$. The thick line represents the gluon propagator in the presence of the background field $\left\langle A_{\mu}\right\rangle$, i.e. with all possible mean field insertions included. The cross represents the

external current $j_{\mu}(x)$, while the circled cross represents the induced current $j_{\mu}^{\text {ind }}(x)$ whose detailed contributions in terms of 2-point and 3-point functions are displayed in (b) (see eq.(2.22)). The contributions of the ghost mean fields are not drawn. Quark, gluon and ghost propagators are represented respectively by straight, wavy and dashed lines.

\section{Fig.2:}

Diagrammatic illustration of eqs. (2.19) for the fermionic field $\langle\psi(x)\rangle$ (a), and its conjugate $\langle\bar{\psi}(x)\rangle(\mathrm{b})$. The thick lines represent quark propagators in the presence of the background gauge field $\left\langle A_{\mu}\right\rangle$. The crosses stand for the external sources $\eta$ and $\bar{\eta}$, and the induced sources are represented explicitly by the appropriate 2-point functions (see eq. $(2.23))$.

\section{Fig.3:}

Lowest order contribution to the off equilibrium fermion propagator (a). The blob represents a gauge field insertion and carry a four momentum $P \sim g T$. Typical radiative corrections are shown in (b). The contributions in (b) are negligible compared to (a) when $k \sim T$, but are of the same order as (a) when $k \sim g T$.

\section{Fig.4:}

Illustration of the approximate equations of motion for $K_{\nu}^{b}(x, y)$, eq. (3.22) (a), and eq. (3.23) (b). Thick lines represent propagators in the presence of the background gauge field $\left\langle A_{\mu}\right\rangle$ (see Fig. 5a for the quark propagator and Fig.6a for the gluon one).

\section{Fig.5:}

Illustration of the approximate equation of motion (3.24) for $S(x, y)$. (a): quark propaga- 
tor with gauge field insertions; (b): corrections induced by the fermionic fields; the thick lines represent propagators in the presence of the background gauge field $\left\langle A_{\mu}\right\rangle$.

\section{Fig.6:}

Illustration of the approximate equation of motion (3.27) for $D(x, y)$. (a):gluon propagator with gauge field insertions; (b): corrections induced by the fermionic fields (only the contribution due to the $K$ function is shown); the thick lines represent propagators in the presence of the background gauge field $\left\langle A_{\mu}\right\rangle$.

\section{Fig.7:}

The induced current $j^{\text {ind }}$ calculated from the 2-point functions obtained in section 3.3. In the first two lines are represented the various contributions to $j^{A}=j_{\mathrm{f}}^{A}+j_{\mathrm{g}}^{A}+j_{G}^{A}+j_{\Omega}^{A}$. In the last two lines are represented, respectively, the contributions $j_{\mathrm{f}}^{\psi}$ and $j_{\mathrm{g}}^{\psi}$ to $j^{\psi}=j_{\mathrm{f}}^{\psi}+j_{\mathrm{g}}^{\psi}$, as obtained by joining the end points $\mathrm{x}$ and $\mathrm{y}$ in Figs.5 and 6. The ghost mean field contributions are not drawn.

\section{Fig.8:}

The induced fermionic source $\eta^{\text {ind }}$ calculated from the 2-point functions obtained in section 3.3, that is, by joining the end points $\mathrm{x}$ and $\mathrm{y}$ in the diagrams in Fig.4. 